\title{
WestVirginiaUniversity
}

THE RESEARCH REPOSITORY @ WVU

Graduate Theses, Dissertations, and Problem Reports

2012

\section{Microstructural Engineering of Porous Cathodes for SOFC Applications}

\author{
Sodith Kumar R. Gandavarapu \\ West Virginia University
}

Follow this and additional works at: https://researchrepository.wvu.edu/etd

\section{Recommended Citation}

Gandavarapu, Sodith Kumar R., "Microstructural Engineering of Porous Cathodes for SOFC Applications" (2012). Graduate Theses, Dissertations, and Problem Reports. 4856.

https://researchrepository.wvu.edu/etd/4856

This Thesis is protected by copyright and/or related rights. It has been brought to you by the The Research Repository @ WVU with permission from the rights-holder(s). You are free to use this Thesis in any way that is permitted by the copyright and related rights legislation that applies to your use. For other uses you must obtain permission from the rights-holder(s) directly, unless additional rights are indicated by a Creative Commons license in the record and/ or on the work itself. This Thesis has been accepted for inclusion in WVU Graduate Theses, Dissertations, and Problem Reports collection by an authorized administrator of The Research Repository @ WVU. For more information, please contact researchrepository@mail.wvu.edu. 
Microstructural Engineering of Porous Cathodes for SOFC Applications

Sodith Kumar R. Gandavarapu

\author{
Thesis submitted to \\ College of Engineering and Mineral Resources \\ at West Virginia University \\ in partial fulfillment of the requirements \\ for the degree of \\ Master of Science \\ in
}

Mechanical and Aerospace Engineering

Edward M. Sabolsky, Ph.D., Chair

John W. Zondlo, Ph.D.

Xingbo Liu, Ph.D.

Department of Mechanical and Aerospace Engineering

\title{
Morgantown, West Virginia
}

2012

Keywords: SOFC, Cathode, LSCF, Porous electrode, Microstructural engineering, Direct foaming, Polyurethane foaming 


\title{
ABSTRACT \\ Microstructural Engineering of Porous Cathodes for SOFC Applications
}

\author{
Sodith Kumar R. Gandavarapu
}

LSCF [ $\left.\left(\mathrm{La}_{0.6} \mathrm{Sr}_{0.4}\right)_{0.98}\left(\mathrm{Co}_{0.2} \mathrm{Fe}_{0.8}\right) \mathrm{O}_{3-\delta}\right]$, a solid oxide fuel cell (SOFC) cathode material was fabricated and foamed through a polymeric in situ foaming process to build an optimum porous architecture. The changes in the porous cathode microstructure with changes in the in situ foaming parameters were qualitatively investigated through back-scattered scanning electron microscope (SEM) imaging. Later, a quantitative analysis of the pore size, shape, area and distribution was completed on the same samples through a computational image analysis program called Image J (National Institute of Health, NIH). Electrochemical testing of the foamed cathode under different processing conditions including the baseline (un-foamed) cathode was performed through electrochemical impedance spectroscopy (EIS) of cathode symmetrical electrolyte-supported cells.

The porous cathode architecture formed through in situ foaming with $70 \%$ solids loading and a polymer precursor composition of 8:4:1 volume ratio (isocyanate: PEG: surfactant) within an terpineol/cellulose printing vehicle yielded the optimum microstructure displaying a substantial decrease in the electrode polarization resistance. It displayed a broad pore size distribution, higher mean pore area and more elongated pore channels with $\sim 40 \%$ and $\sim 50 \%$ less polarization than the baseline cell at $750^{\circ} \mathrm{C}$ and $800^{\circ} \mathrm{C}$, respectively. These measurements were completed at open circuit voltage $(\mathrm{OCV}), 100 \mathrm{~mA}$ and $300 \mathrm{~mA}$ loading. Electrochemical Impedance Spectroscopy (EIS) testing for this cathode displayed $\sim 0.08 \Omega \mathrm{cm}^{2}$ - polarization at $800^{\circ} \mathrm{C}$ (at OCV) and $\sim 50 \%$ increase in maximum power density with the foamed cathode over the baseline. Further improvements in the foamed cathode performance were obtained through the nano-catalyst incorporation into this microstructure. Platinum $(\mathrm{Pt})$ nano-catalyst was impregnated into the microstructure using water based precursor $\left(\mathrm{H}_{2} \mathrm{PtCl}_{6} \cdot 6 \mathrm{H}_{2} \mathrm{O}\right)$ solution; the interconnected porosity permitted the efficient infiltration of the solution throughout the bulk of the microstructure using a lower number of processing steps than the baseline (unfoamed) microstructure (per infiltration cycle). Also, a homogeneous dispersion of the nano-catalyst across the foamed cathode led to higher power densities, which is further reported in this study. 


\section{DEDICATION}

This thesis is dedicated to my parents who has been my strength all through. 


\section{ACKNOWLEDGEMENT}

I would like to take this opportunity to thank several people who played a vital role in the successful completion of this thesis. First and foremost, my utmost gratitude to my advisor and committee chair person Dr. Edward M. Sabolsky, whose guidance all through the thesis was inspiringly learning experience which made this dissertation possible. His scientific intuition led me grow as an engineer to understand the science behind what I do. I sincerely thank Dr. John W. Zondlo and Dr. Xingbo Liu for reviewing my thesis and being in my advisory committee.

I gratefully acknowledge the financial support for this thesis by National Energy Technology Laboratory (WV), US-Department of Energy. I extend my greatest gratitude to Dr. Gerdes for his invaluable support and guidance all through, which added a great value to this work. My heart full gratitude is extended to Dr. Thomas Kalapos, Dr. Chunchuan Xu, James Poston, Dr. Shiwoo Lee and Dr. Gaetano Impoco for their greatly appreciated contribution to this work.

The support and encouragement from my lab mates during my stay as graduate research assistant at WVU is greatly appreciated. The support and love extended by my family Mohan Talamati (uncle), Jayanthi Talamati (aunt), Vahini Talamti and Krishna Talamati (cousins) for making me feel at home while being thousands of miles away from my parents. I thank from the bottom of my heart all my friends Ravi, Manoj, Sriram, Praneeth, Sai Bharath and Nani for being a great support all through my stay in USA without whose presence my journey would not be as smooth and fun loaded as it is now.

This thesis work was funded by NETL-RUA (US-DOE), under contract no. RES1000023. 


\section{DISCLAIMER}

This report was prepared as an account of work sponsored by an agency of the United States Government. Neither the United States Government nor any agency thereof, nor any of their employees, makes any warranty, express or implied, or assumes any legal liability or responsibility for the accuracy, completeness, or usefulness of any information, apparatus, product, or process disclosed, or represents that its use would not infringe privately owned rights. Reference herein to any specific commercial product, process, or service by trade name, trademark, manufacturer, or otherwise does not necessarily constitute or imply its endorsement, recommendation, or favoring by the United States Government or any agency thereof. The views and opinions of authors expressed herein do not necessarily state or reflect those of the United States Government or any agency thereof. 


\section{TABLE OF CONTENTS}

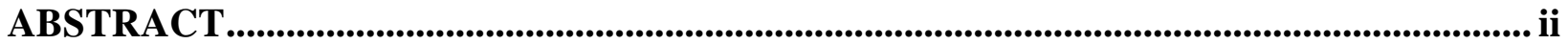

ACKNOWLEDGEMENT............................................................................................................ v

Table of Contents........................................................................................................................................... vii

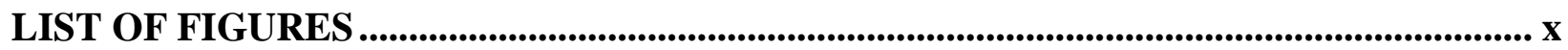

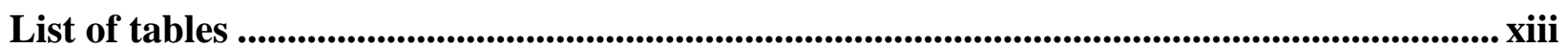

Chapter 1: INTRODUCTION ........................................................................................... 14

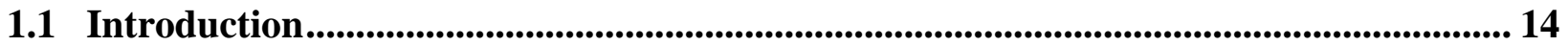

1.2 Objective .......................................................................................................................... 17

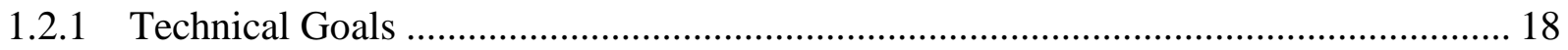

1.2.2 Desired attributes for microstructure and processing ............................................... 18

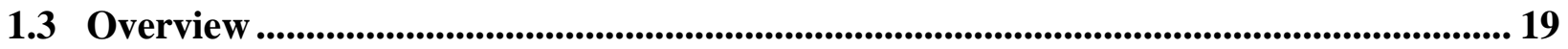

Chapter 2: Background ................................................................................................................. 20

2.1 Solid Oxide Fuel Cells (SOFC) ....................................................................................................... 20

2.2 Working Principle ..................................................................................................................... 20

2.3 Components of SOFC .................................................................................................................... 24

2.4 Triple Phase Boundary (TPB) ……............................................................................................. 24

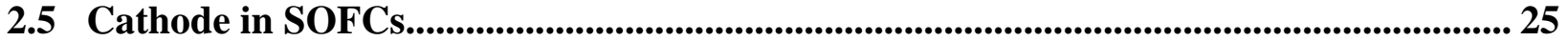

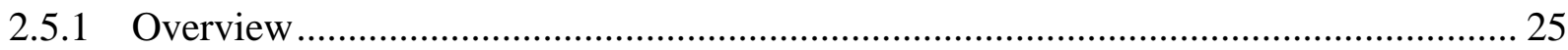

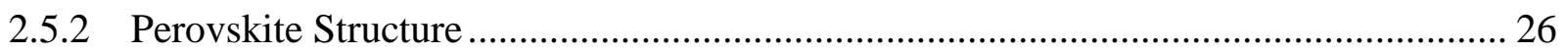

2.5.3 Lanthanum Manganite based cathodes ...................................................................... 28

2.5.4 Lanthanum based Ferro Cobaltite (LSCF) cathodes.................................................... 28 
2.7 Typical porous structure processing - SOFC cathodes ................................................... 32

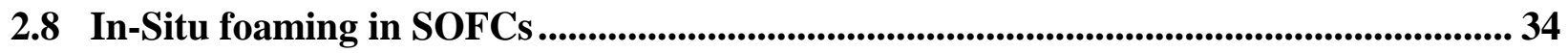

2.9 Nano-catalyst impregnation ...................................................................................... 35

Chapter 3: In-situ foaming and effect of processing parameters on microstructure ...... 37

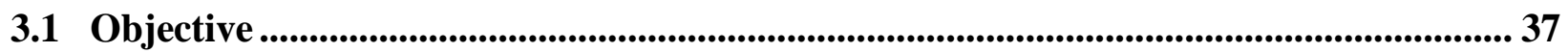

3.2 Experimental ............................................................................................................................... 37

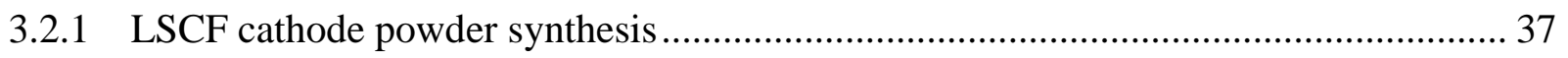

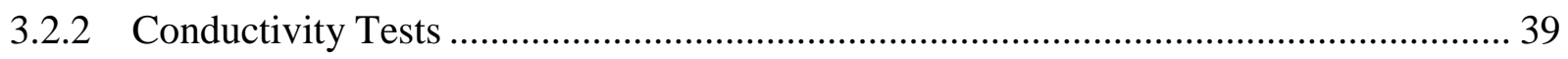

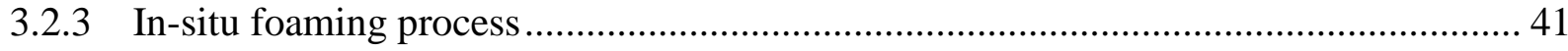

3.2.4 Computational Image Analysis -Image J ................................................... 42

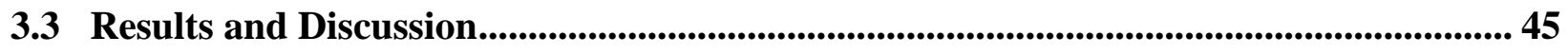

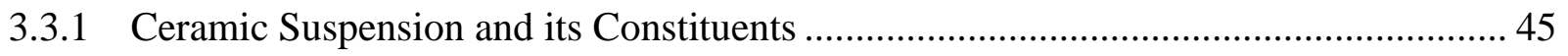

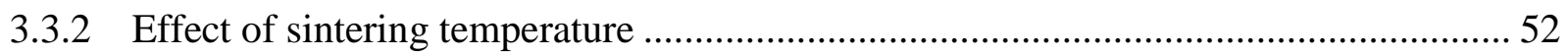

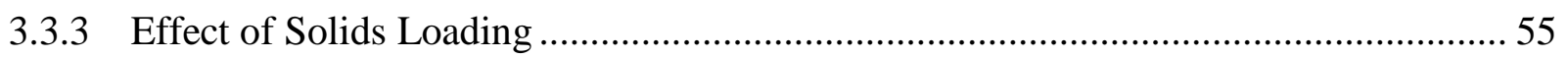

3.3.4 Effect of polymer precursor composition ....................................................... 59

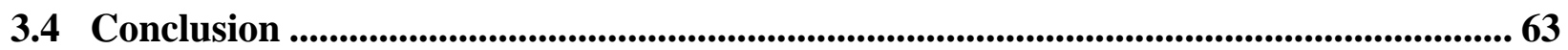

Chapter 4: In situ foaming of cathode over electrolyte and electrochemical testing ....... 64

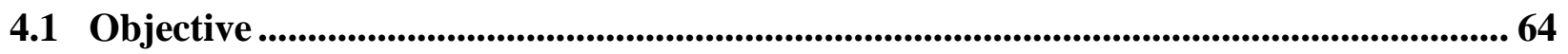

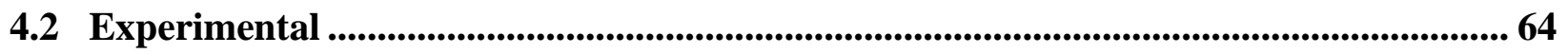

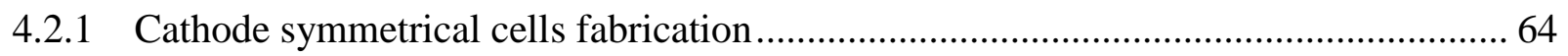

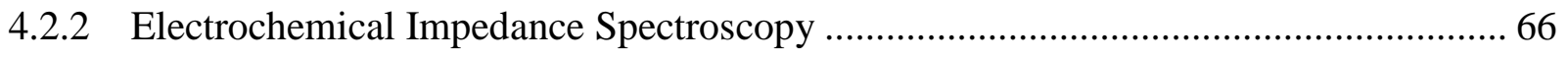

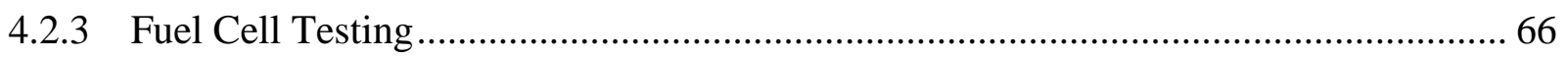

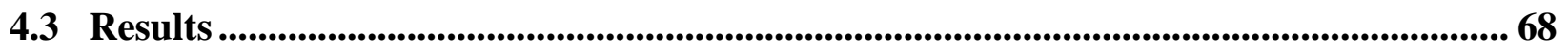


4.3.1 Effect of polymer precursors composition on foamed cathode over the YSZ electrolyte 68

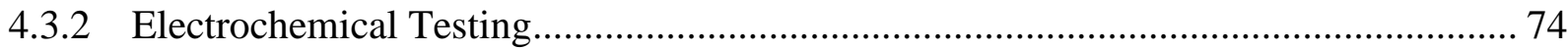

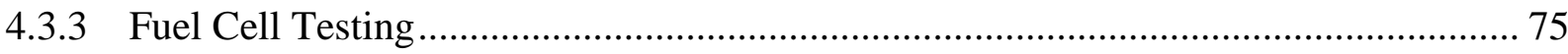

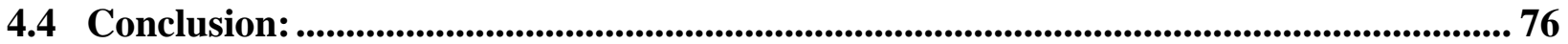

Chapter 5: Effect of foamed cathode structure on nano-catalyst infiltration .................... 77

5.1 Back ground and Objective...................................................................................... 77

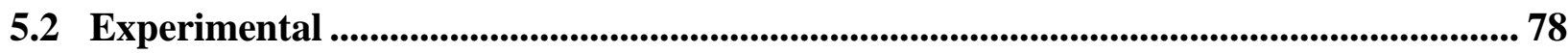

5.2.1 Infiltration of Platinum nano catalyst …………………………………………. 78

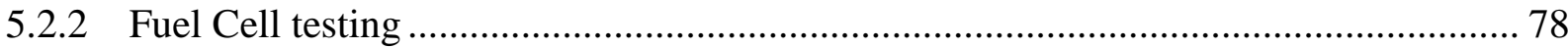

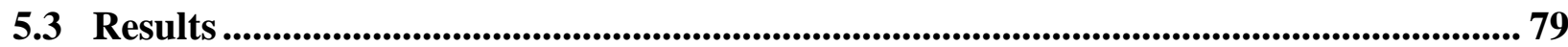

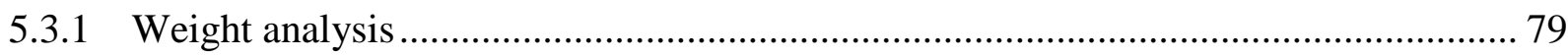

5.3.2 SEM and EDS analysis ........................................................................................... 81

5.3.3 Fuel cell testing ..................................................................................................... 83

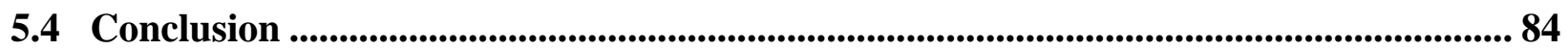

Chapter 6: Conclusions and Future scope ……............................................................................ 85

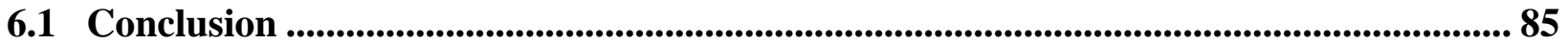

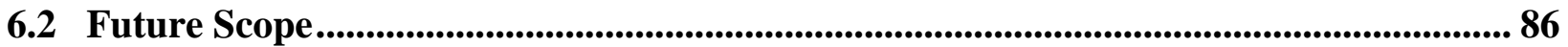

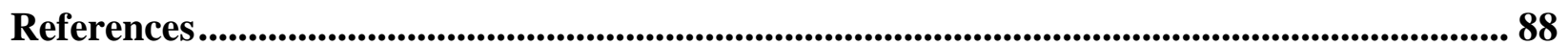

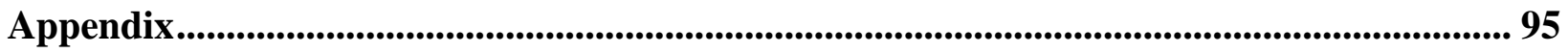




\section{LIST OF FIGURES}

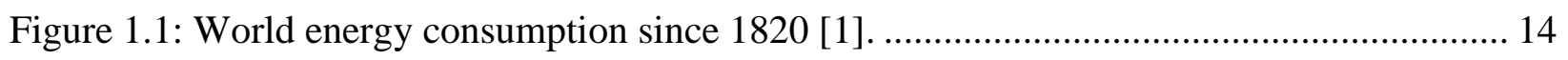

Figure 1.2: Projected transportation fuel cell system cost [8] ............................................ 16

Figure 1.3: Development in commercialization of fuel cell technologies [10]. ........................ 16

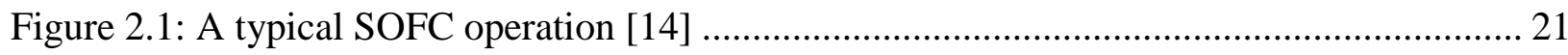

Figure 2.2: A simplified schematic of a TPB in a SOFC electrode [15] ................................ 25

Figure 2.3: Typical cubic perovskite structure [28] ........................................................... 27

Figure 2.4: Illustration of different techniques for porous ceramics [45]............................... 32

Figure 2.5:Basic polymerization reaction in direct foaming [47] ........................................ 35

Figure 3.1: XRD pattern of LSCF powder at different calcination temperatures...................... 38

Figure 3.2: Sintering study of the 2\% A-site deficient samples. ........................................... 39

Figure 3.3: Electronic conductivity versus isothermal temperature data for A-site deficient

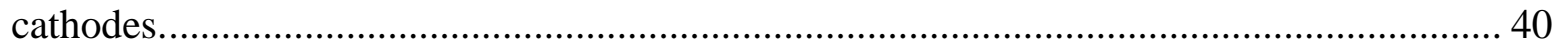

Figure 3.4: (Left) Back scattered SEM image of a low viscous epoxy infiltrated foamed LSCF sample which is binarized (right) for phase differentiation computational image analysis.. 44

Figure 3.5: Method for deriving circularity factor from image analysis. 45

Figure 3.6: Viscosity Measurements LSCF with ink vehicle with different volume ratios of solid to solvent. 48

Figure 3.7: Viscosity measurements of LSCF + polymer precursors' mixture (8:4:1) with different solvents at constant 2:5 vol ratio of solids to solvent, terpineol and ink sintered at $1150^{\circ} \mathrm{C}$.

Figure 3.8: Pore count versus pore area of sample foamed with $70 \%$ solids loading with change inn solvents sintered at $1150^{\circ} \mathrm{C}$. 51

Figure 3.9: Pore count versus pore area of sample foamed with $70 \%$ solids loading with change in isocyantaes sintered at $1150^{\circ} \mathrm{C}$. (A-polymethylene polyphenylisocyanate; B- Isophorone

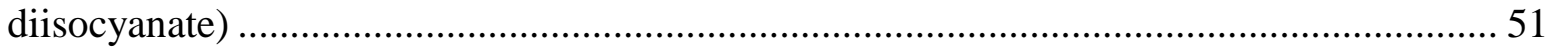

Figure 3.10: Effect of sintering temperature on microstructure of bulk foamed cathode with $70 \%$ and 50\% solids loading at 8:4:1 polymer precursor's composition away from the YSZ substrate. 
Figure 3.11: Back scattered SEM image of bulk foamed cathode with $70 \%$ solids loading and 8:4:1 polymer precursor's composition away from the YSZ substrate, sintered at $1350^{\circ} \mathrm{C}$ and $1050^{\circ} \mathrm{C}$

Figure 3.12: Effect of solids loading on microstructure of bulk foamed cathode with 8:4:1 polymer precursor's composition - away from the YSZ substrate, sintered at $1150^{\circ} \mathrm{C}$ 57

Figure 3.13:Back scattered SEM image of bulk foamed cathode with 8:4:1 polymer precursor's composition at $30 \mathrm{vol} \%$ and $70 \mathrm{vol} \%$ solids loading, sintered at $1150^{\circ} \mathrm{C}$. 58

Figure 3.14: Pore area distribution (pore count versus pore area) as a function of solids loading for LSCF samples foamed with 8:4:1 composition and sintered at $1150^{\circ} \mathrm{C}$. 59

Figure 3.15: Effect of polymer precursor's composition on microstructure of bulk foamed cathode with $70 \%$ solids loading away from the YSZ substrate, sintered at $1150^{\circ} \mathrm{C}$. 61

Figure 3.16: Back scattered SEM image of bulk foamed cathode with 8:4:1, 8:4:2 and 12:2:1 polymer precursor's composition at $70 \mathrm{vol} \%$ solids loading, sintered at $1150^{\circ} \mathrm{C}$. 62

Figure 3.17:Pore area distribution (pore count versus pore area) as a function of precursor composition for LSCF samples foamed with $70 \%$ solids loading and sintered at $1150^{\circ} \mathrm{C}$. 63

Figure 4.1: Schematic drawing of an electrolyte supported cathode symmetrical cell. 66

Figure 4.2: Configuration of electrolyte supported full SOFC. 67

Figure 4.3: Effect of polymer precursor's composition on foamed cathode with $70 \%$ solids loading over the YSZ substrate, sintered at $1150^{\circ} \mathrm{C}$. 70

Figure 4.4: Across the thickness of the cathode (nearer and farther from electrolyte), effect of polymer precursor's composition on foamed cathode with $70 \%$ solids loading over the YSZ substrate, sintered at $1150^{\circ} \mathrm{C}$. 72

Figure 4.5: Back-scattered SEM micrograph of LSCF cathode film deposited and in-situ foamed on YSZ substrate at a 70\% solids loading and 8:4:1 polymer precursor's composition (sintered at $1150^{\circ} \mathrm{C}$ ) shown at lower and higher magnifiacations. 73

Figure 4.6: EIS spectrum of electrolyte-supported cathode symmetrical cells through EIS at $750^{\circ} \mathrm{C}$ and $800^{\circ} \mathrm{C}$ at $\mathrm{OCV}, 100 \mathrm{~mA}$ and $300 \mathrm{~mA}$ loadings. 75

Figure 4.7: $\mathrm{I}-\mathrm{V}$ and Power density curves of foamed and baseline cells at $800^{\circ} \mathrm{C}$ with moist $\mathrm{H}_{2}$ as fuel. 76

Figure 5.1: a) Amount of catalyst in cathode per infiltration step (calcined at $850^{\circ} \mathrm{C} / 1 \mathrm{~h}$ ), b) The amount of precursor solution could be added per infiltration step (no calcination) 80 
Figure 5.2: SEM images of fractures surface of the cross section of infiltrated foamed and baseline cathodes in a) top (away from electrolyte) b) middle and c) bottom (at electrode-

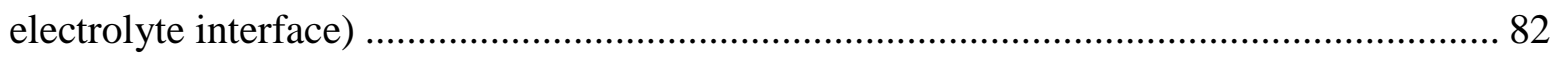

Figure 5.3: $\mathrm{I}-\mathrm{V}$ and power density curves of SOFC button cells with impregnated foamed and baseline LSCF cathodes measured at $800^{\circ} \mathrm{C}$ with $\mathrm{H}_{2}$ fuel............................................ 84 


\section{LIST OF TABLES}

Table 3.1: Effect of solvents and polymer precursor on the microstructure of bulk foamed cathode with $70 \%$ solids loading away from the YSZ substrate, sintered at $1150^{\circ} \mathrm{C} \ldots \ldots \ldots . . . .50$

Table 5.1: Atom $\%$ of corresponding element (farther from eelctrolyte) ................................. 83

Table 5.2: Atom $\%$ of corresponding element (midddle zone) .............................................. 83

Table 5.3: Atom\% of corresponding element (cathode/electrolyte interface zone) ................... 83 


\section{Chapter 1: INTRODUCTION}

\subsection{Introduction}

Clean energy production has gained importance due to the increase in the environmental pollution to an alarming level. The exponential growth in usage of power has also triggered a possible extinction of fossil fuels in the near future, which leads to the growing need for alternative energy technologies. There is a continuous steep rise in world energy consumption in all sectors by nearly 50 times since 1820 to 2000 . This surge of energy consumption is generated from different sources since 1820 (clearly depicted in Fig.1.1 by Gail Tverberg [1]). The world's energy consumption is estimated to be 770 quadrillion Btu by 2035 which is 53\% higher than 505 quadrillion Btu in 2008 [2].

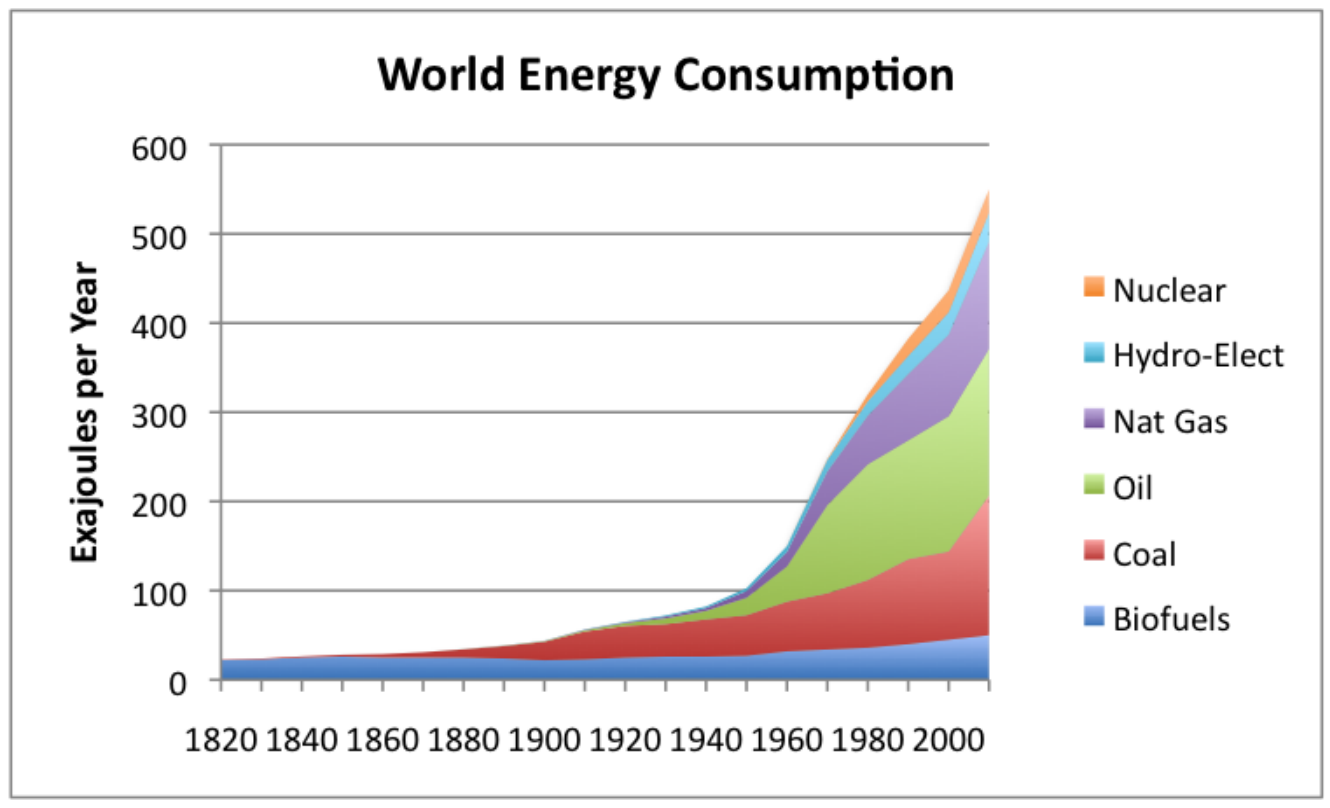

Figure 1.1: World energy consumption since 1820 [1].

The USA, Russia and China together consume $41 \%$ of the world's total energy while contributing only $31 \%$ of total production. The USA stands out by leading in terms 
of consumption by almost 3 times that of China, which is second to it in consumption [3]. The rise in energy consumption resulted in proliferation of greenhouse gases (GHG) in the atmosphere as a result of emissions from fossil fuel combustion, the ramification of which is an increase in global warming. Around $90 \%$ of total commercial energy being generated in the world is through fossil fuels while carbon dioxide $\left(\mathrm{CO}_{2}\right)$ constitutes to more than $80 \%$ of GHG emissions [4]. An unprecedented amount of global $\mathrm{CO}_{2}$ emissions, about 31.6 gigatonnes (Gt), was recorded in 2011 as reported by International Energy Agency [5]. All data reported to date clearly displays the urgency in the development of a clean energy generation from alternative energy sources. Research on fuel cells as a potential solution for clean power generation has prominently focused upon the use of hydrogen as fuel over the past few decades. The exhaust of fuel cells with hydrogen as fuel is just water vapor making fuel cells an able alternative for clean energy generation. It is only since the 1960s the fuel cells gained importance as a potential alternative after the United States space program adopted fuel cells into spacecraft over risky and costly nuclear and solar power sources. Fuel cells were used in power generation for Gemini and Apollo spacecraft as well for producing water in space shuttles [6]. The commercialization of fuel cells requires great attention and push, to advance its eminent existence as stationary power generators contributing to a great extent in reduction of the carbon foot print by intruding into the domestic and industrial power sector. In the process of supporting the development of such technology, the Department of Defense (DoD) and Department of Energy (DoE) accounts for a major share in funding. It is an approximate estimation that DoD has spent around \$44-60 million in FY10 for research and development in fuel cells [7]. The DoE in its accomplishments and progress sheet reported that the research advancements in this field funded by DoE has yielded in $80 \%$ reduction of cost of automotive fuel cells since 2002 from $\$ 275 / \mathrm{kW}$ in 2002 to $\$ 49 / \mathrm{kW}$ in 2011 as shown in Fig.1.2 [8]. DoE also reported a 25\% improvement in the power density of SOFC systems which in turn leads to $30 \%$ reduction in stack volume, stating the improvement in performance of stationary fuel cells in their 2010 annual merit review proceedings [9]. The funding and support from DoE has been very extensive and has resulted in 313 patents with more than 60 emerging and 30 commercial 
technologies leading to $\$ 200$ million in industrial investment and revenues from around $\$ 70$ million of funding [10].

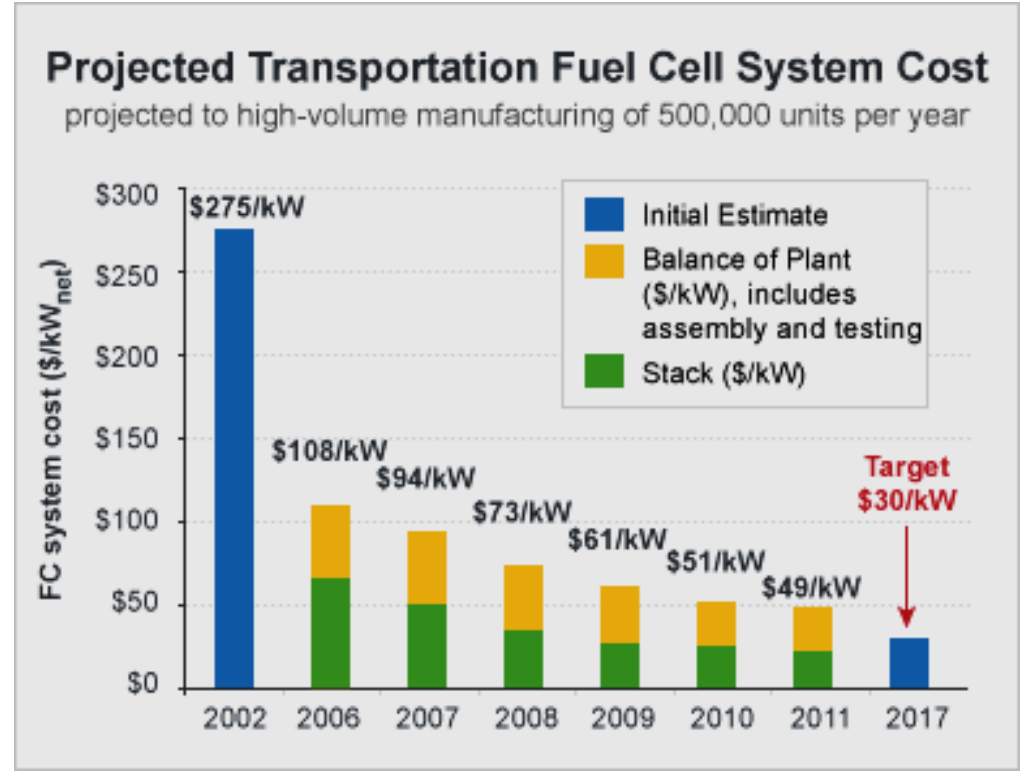

Figure 1.2: Projected transportation fuel cell system cost [8].

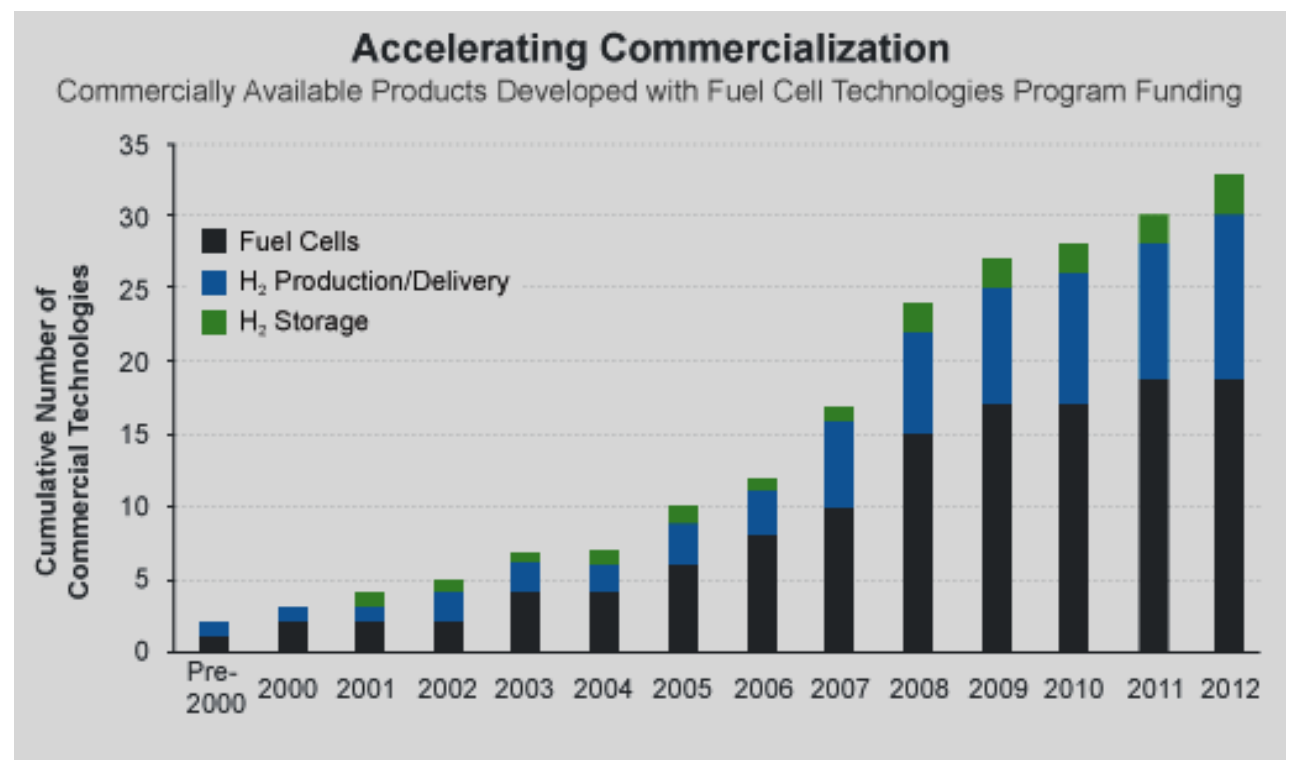

Figure 1.3: Development in commercialization of fuel cell technologies [10]. 
The research and development as stated above is making fuel cell technology, especially the stationary fuel cell industry, a fast-growing, clean energy alternative. The Clean Technica [11] reported that around 9000 stationary fuel cell units were sold in 2010, which is $60 \%$ higher than 2009 and expected to be 1.2 million by 2017 . The major emphasis has been demonstrated on polymer electrolyte membrane fuel cells (PEM). Though the PEM fuel cell is advantageous for its ability to operate at low temperatures, its sensitivity to poisoning by carbon monoxide $(\mathrm{CO})$ results in the strict need of pure hydrogen as fuel. Hence, the technology is limited due to constrained availability of pure hydrogen with its associated problem of storage and production and its high cost. Solid oxide fuel cells (SOFCs) acquired an appreciable scale of feasibility for being widely used as the fuel cell technology of popular interest because of its fuel versatility and operation at intermediate temperatures. The overall performance of SOFC as a system is highly limited to the oxygen $\left(\mathrm{O}_{2}\right)$ reduction and transfer of its ions into the electrolyte, which later reacts with the hydrogen (fuel) releasing electrons. The cathode is responsible for the $\mathrm{O}_{2}$ reduction and $\mathrm{O}^{2-}$ incorporation, which clearly indicates the importance of cathode functioning on the whole SOFC system.

\subsection{Objective}

The objective of this work is to investigate engineered solid oxide fuel cell (SOFC) cathode microstructures that are tailored to provide a high triple-phase boundary (TPB) concentration, low resistance to gas diffusion, and optimal architecture for nanocatalyst impregnation. The goal is to produce a foam-like cathode structure with a broad pore size distribution and engineered mesoporous structure within the legs of an open foam network. This configuration will provide a greatly enhanced TPB concentration

while retaining the open pore structure for optimal gas flow and liquid impregnation of the cathode active area. 


\subsubsection{Technical Goals}

- Attaining an improved porous architecture of the cathode through microstructural engineering.

- Correlation of the processing parameters with the microstructural changes through $2 \mathrm{D}$ image analysis.

- Deriving the right composition of the processing parameters for the improved microstructure of the cathode and confirming their credibility through electrochemical testing of the developed microstructure in comparison with the baseline.

- Investigating the effectiveness of the improved microstructure for nano-catalyst impregnation.

\subsubsection{Desired attributes for microstructure and processing}

The following is a list of microstructural attributes of a cathode structure required for enhanced performance of the SOFC system:

- A porous cathode with $30-60 \%$ porosity through controlled in-situ pore forming strategies.

- The gradient in pore size across the thickness (cross section) of the cathode where the interface has a large number of small pores for better adhesion with high triple phase boundary concentration, while the surface has larger pores assisting in gas penetration and diffusion.

- Porous cathode architecture with tortuous and interconnected porosity.

- A cathode architecture that supports a well-diffused, uniform surface area yielding a homogeneous nano-catalyst distribution upon impregnation. 


\subsection{Overview}

This work will incorporate a polymeric in situ foaming process to build the porous architecture in the $\operatorname{LSCF}\left[\left(\mathrm{La}_{\mathrm{x}} \mathrm{Sr}_{1-\mathrm{x}}\right)_{\mathrm{s}}\left(\mathrm{Co}_{\mathrm{y}} \mathrm{Fe}_{1-\mathrm{y}}\right)_{1-\mathrm{s}} \mathrm{O}_{3-\delta}\right]$ cathode. The porosity distribution, structural architecture and reaction kinetics were distinctly controlled by varying several processing parameters, such as precursor composition, solvent, solids loading and catalyst concentration. Various combinations of the above-discussed parameters were investigated allowing for optimal control of the porous structure, pore volume distribution and porosity levels. The pore size, shape, distribution and orientation were characterized through computational image analysis of scanning electron microscope (SEM) images. The measured microstructural parameters were related to the processing variables and electrochemical testing. The in situ foaming process was modified in order to deposit the LSCF foamed cathodes onto electrolyte-supported cells to build a workable thick film fuel cell cathode. Nano-catalyst was infiltrated through the foamed cathode while maintaining a homogeneous dispersion across the microstructure (as characterized by SEM imaging). The results obtained from current-voltage (I-V) testing, along with electrochemical impedance spectroscopy (EIS), were used to draw final processing-microstructure-properties correlations. 


\section{Chapter 2: BACKGROUND}

\subsection{Solid Oxide Fuel Cells (SOFC)}

A solid oxide fuel cell is an electrochemical device which converts chemical energy into electrical energy and heat. The SOFC usually functions at higher temperatures, such as $600-1000^{\circ} \mathrm{C}$. The operation of SOFCs at higher temperatures leads to highly efficient conversion to power, internal reforming and useful heat as a byproduct of the co-generation. SOFCs are capable of generating power with high electrical efficiencies ranging near 55\%, and this value can be escalated up to $70 \%$ and $90 \%$ in case of hybrid power generation with gas turbines and combined/power generation systems, respectively [12]. Solid oxide fuel cells acquired an appreciable scale of feasibility for being widely used in various applications due to its fuel adaptability and higher efficiencies over polymer or carbonate electrolyte fuel cells. The solid electrolyte used for SOFCs is in general a ceramic material such as yttria-stabilized zirconia (YSZ). The dense solid oxide electrolyte is an ionic conductor, which is sandwiched between porous electrodes each of which display mixed ionic-electronic conducting capabilities.

\subsection{Working Principle}

The porous cathode is fed with air while a gaseous fuel is supplied to the anode. The oxygen molecules from air adsorb onto the solid cathode, where the molecular oxygen undergoes a reduction process with the available electrons on the electrode surface. This reduction process results in the formation of oxygen ions $\left(\mathrm{O}^{2-}\right)$. These oxygen ions are then incorporated into the electrolyte phase and diffuse through the solid electrolyte to the electrolyte/anode interface. At this location, the oxygen ions react with the fuel, which is typically hydrogen $\left(\mathrm{H}_{2}\right)$, liberating water vapor and free electrons. The stream of these free electrons is passed through the load and back to the cathode where they reduce the oxygen molecules to oxygen ions [13]. The illustrative working principle 
of a SOFC is shown below in Fig. 2.1 and the electrochemical reactions are listed below [18].

At the cathode:

$$
1 / 2 \mathrm{O}_{2}(\mathrm{~g})+2 \mathrm{e}^{-} \rightarrow \mathrm{O}^{2-}
$$

At the anode:

$$
\begin{aligned}
& \mathrm{H}_{2}(\mathrm{~g})+\mathrm{O}^{2-} \rightarrow \mathrm{H}_{2} \mathrm{O}(\mathrm{g})+2 \mathrm{e}^{-} \\
& \quad \text { Overall Reaction: } \\
& \mathrm{H}_{2}(\mathrm{~g})+1 / 2 \mathrm{O}_{2}(\mathrm{~g}) \rightarrow \mathrm{H}_{2} \mathrm{O}(\mathrm{g})
\end{aligned}
$$

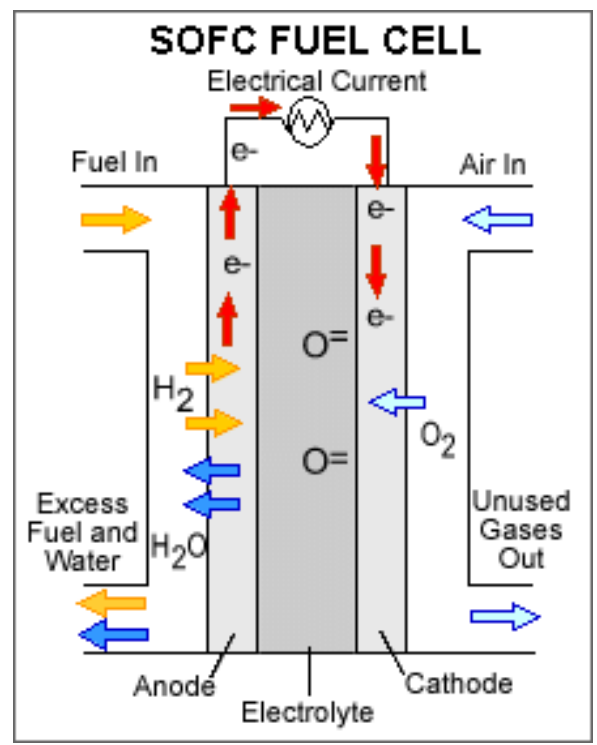

Figure 2.1: A typical SOFC operation [14]

The theoretical cell voltage under open circuit conditions $\left(\mathrm{E}_{\mathrm{r}}\right)$ in SOFCs can be determined using the Nernst equation [19].

$$
E_{r}=\left(\frac{R T}{2 F}\right) \ln K_{T}-\left(\frac{R T}{4 F}\right) \ln \frac{\left(p_{H_{2} O}\right)^{2} p_{O}}{\left(p_{H_{2}}\right)^{2} p_{O_{2}}}
$$

The actual cell voltage under operating conditions is always less than the theoretical Nernst potential. As under operating conditions, SOFCs are subjected to the influence of three major polarization losses: ohmic polarization $\left(\mathrm{V}_{\mathrm{ohm}}\right)$, concentration 
polarization $\left(\mathrm{V}_{\text {conc. }}\right)$ and activation polarization $\left(\mathrm{V}_{\text {act }}\right)$ [19]. The actual cell voltage (E (i)) is given by the following equation [24].

$$
E(i)=E_{r}-V_{\text {ohm }}-V_{\text {conc }}-V_{\text {act }} \quad 2.5
$$

Ohms law indicates that the resistance offered by the material affects the electrical charge transfer through any conductor. The total ohmic resistance or polarization is attributed to the combined electron and ionic mobility within the electrodes and electrolyte materials [21].

The migration of the reactant and product gases through the electrodes is dependent on the diffusivity and microstructural properties of the electrode [22]. The physical resistance offered by electrodes for the gas molecule migration through them results in the concentration polarization [21].

The electrochemical reaction occurring in electrodes is a result of a series of multiple steps. The rate of the reaction is dependent on the slowest process of all the steps involved. The net current density is dependent on the reaction rate, which leads to voltage loss known as activation polarization or overpotential [21].

The understanding of the reaction mechanism that contributes to the performance of a SOFC as a system is a very crucial step. The oxygen reduction mechanism (ORR) that takes place within the cathode is considered to be a difficult reaction to get activated, which explains its contribution to the activation polarization in a SOFC operation. Hence, development of an optimal cathode plays a vital role in the enhancement of SOFC performance. There are three different pathways that are generally considered through which the oxygen reduction takes place [54]. They are:

a) Electrode surface path - oxygen diffusion followed by adsorption of oxygen molecules onto the electrode surface. The ionized oxygen atom/ion diffuses along 
the surface of the electrode to the triple phase boundary where complete ionization takes place while the oxygen ions are transported into electrolyte.

b) Bulk path - the diffused and adsorbed oxygen dissociates and ionizes which is followed by ions being incorporated into the cathode. The oxygen ion then travels through the cathode and enter the electrolyte.

c) Electrolyte surface path - it is very similar to surface path except the travel of the ionized oxygen is directly transported to the electrolyte instead of traveling along the cathode surface or through the cathode.

The oxygen reduction will be processed through one or combination of the above said pathways. The oxygen reduction reaction can be represented using Kroger-Vink notation as shown below, which includes the multiple intermediate steps of surface diffusion, dissociation and charge transfer of oxygen [55].

$$
\begin{array}{ll}
\mathrm{O}_{2}(\mathrm{~g})+4 \mathrm{e}^{-}+2 \mathrm{~V}_{\mathrm{o}} \rightarrow 2 O_{o}^{X} & 2.6 \\
& \\
1 / 2 \mathrm{O}_{2} \rightarrow \mathrm{O}_{\mathrm{ad}} & 2.6 .1 \\
\mathrm{O}_{\mathrm{ad}}+\mathrm{e}^{-} \rightarrow O_{a d}^{-} & 2.6 .2 \\
O_{a d}^{-} \rightarrow O_{T P B}^{-} & 2.6 .3 \\
O_{T P B}^{-}+\mathrm{e}^{-}+V_{O} \rightarrow O_{o}^{X} & 2.6 .4
\end{array}
$$

The reaction mechanism is a complex phenomenon. There are many factors such as material properties, microstructure, partial pressure of oxygen, etc. that affects these steps and contribute to the polarization. Adler [56] in his review of factors governing the oxygen reduction process gave an insight on this area. This study concludes that a given microstructure usually forms a tradeoff between high surface area (that needs lower sintering temperature) and better electrode/electrolyte contact that requires (higher sintering temperature). Physical processes like oxygen adsorption, charge transfer and electrochemical kinetics at an interface usually limit a cathode's reaction mechanism. Improvement in any of these areas will contribute to the enhancement in performance. 
It is learned that the cathode microstructure with higher surface area of the active region by interconnected (tortuous) porosity and open pore structure, leads to better adsorption of diffused oxygen along with improved charge transfer. Addressing this issue is the central focus of this work.

\subsection{Components of SOFC}

The basic components of SOFC are cathode, anode and electrolyte. Based on the component that supports the structure of the full cell, the SOFCs are briefly divided into anode, cathode and electrolyte-supported cells. An SOFC with the anode being the thickest component is termed as an anode-supported cell; similarly, cells with the bulk of the material composed of cathode or electrolyte materials lead to the cathode- and electrolyte-supported cell architectures, respectively. The electrolyte-supported cell is considered to offer less electrode concentration polarization, but high ohmic loss. The cathode- and anode-supported cells offer low ohmic loss, but depending upon the thickness of the electrodes, the concentration polarizations may be elevated [17].

\subsection{Triple Phase Boundary (TPB)}

The hydrogen oxidation reaction (HOR) at the anode and the oxygen reduction reaction (ORR) at the cathode are both typically limited to their respective electrode/electrolyte interfaces, commonly known as the triple phase boundary (TPBs). The TPB is a point where the electron conductor, gas and ionic conductor coexist; this is the location where electrode, air/fuel and electrolyte meet. Since the TPBs are the regions where the basic and vital catalytic oxidation and reduction reactions occur, the reaction kinetics at TPBs significantly affect the SOFC performance. A simplified illustration of the TPB cathode/electrolyte interface is shown in Fig. 2.2. 
Three Phase Boundary (TPB)

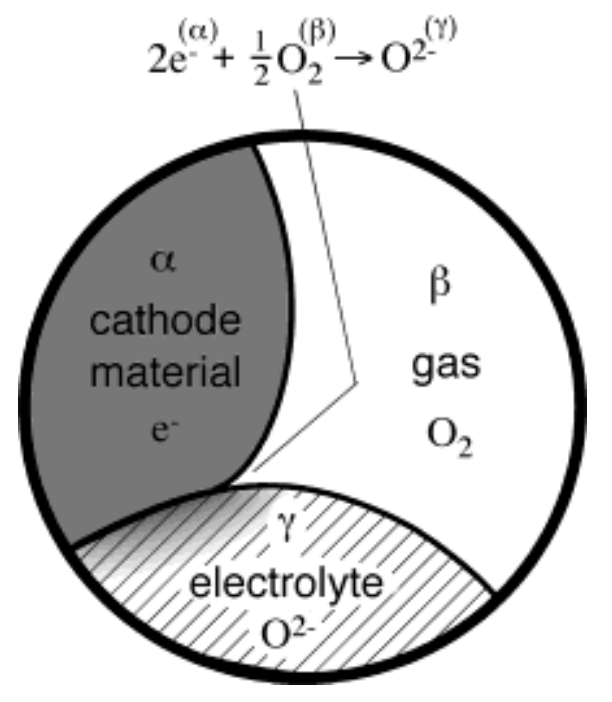

Figure 2.2: A simplified schematic of a TPB in a SOFC electrode [15].

Efforts to infiltrate various nanomaterial versions of any catalyst that enhance the catalytic properties of the electrode, such as adsorption and electrochemical reaction, along with the multiplication of the pore spaces for gas have yielded appreciably improved reaction kinetics along with the SOFC performance [16].

\subsection{Cathode in SOFCs}

\subsubsection{Overview}

The SOFC is generally operated at higher temperatures $\left(600-1000^{\circ} \mathrm{C}\right)$, which is one of the main issues related to this technology and is being widely addressed by many researchers. The most widely used cathode materials in SOFC applications are perovskite-structured, electronically conductive strontium-doped lanthanum manganite $\mathrm{LaSrMnO}_{3}$ (LSM) and mixed-ionic and electronic conductive lanthanum cobaltite and ferrite - $\left(\mathrm{La}_{\mathrm{x}} \mathrm{Sr}_{1-\mathrm{x}}\right)_{\mathrm{s}}\left(\mathrm{Co}_{1-\mathrm{y}} \mathrm{Fe}_{\mathrm{y}}\right) \mathrm{O}_{3}(\mathrm{LSCF})$ materials [23]. The major electrochemical reaction that occurs at the cathode is the oxygen reduction to oxygen ions. It is very evident that a cathode should possess high electronic and ionic conductivity, as well as, an optimum porous structure that supports oxygen gas diffusivity. The thermal, chemical 
and physical compatibility with the electrolyte under the high operating temperatures is also a vital property for cathode [26]. Since the oxygen reduction process is confined to triple phase boundaries at the electrode/electrolyte interface, any defect such as delamination of cathode from electrolyte mitigates the active area for electrochemical reaction. This means that a decrease in the oxygen reduction kinetics will significantly limit the performance of the overall SOFC system. Hence, the cathodes which are mixed ionic and electronic conductive (MIEC), such as LSCF, have a theoretically higher effective TPB area due to the dual conduction behavior. This dual nature allows oxygen reduction to happen through the cathode structure unlike only electronic conductive cathodes such as LSM [24]. MIECs also provide an advantage of allowing bulk diffusion path within the solid cathode instead of limiting all ionic conduction to the surface. This is found to be a significantly limiting feature of purely electronic conductive materials such as LSM. The bulk diffusion within the cathode crystal structure involves oxygen ionization at the cathode surface with further incorporation and diffusion through the cathode solid material. The oxygen ion must enter the electrolyte at some point within the cathode structure, but MIEC allows for parallel surface and bulk diffusion paths for this process. The effectiveness of the bulk diffusion path is highly dependent on the ratio of surface diffusion coefficient to bulk diffusion coefficient. The mechanism is also dependent upon the ratio of available surface area (or grain boundary area) to bulk volume, where low particle sizes will lead to a high level of surfaces per volume, thus surface diffusion and high levels of hydrogen adsorption would be enhanced within the system [27].

\subsubsection{Perovskite Structure}

The perovskite structure is generally represented as $\mathrm{ABO}_{3}$, where $\mathrm{A}$ and $\mathrm{B}$ in this formula are cations whereas $\mathrm{O}$ is the anion. In general, A-cations are considered to be smaller with lower valence that are 12-fold coordination site, while B-cations are larger with higher valence and are 6-fold coordinated with oxygen anions. The typical structure of ideal cubic perovskite structure is shown in Fig. 2.3. 


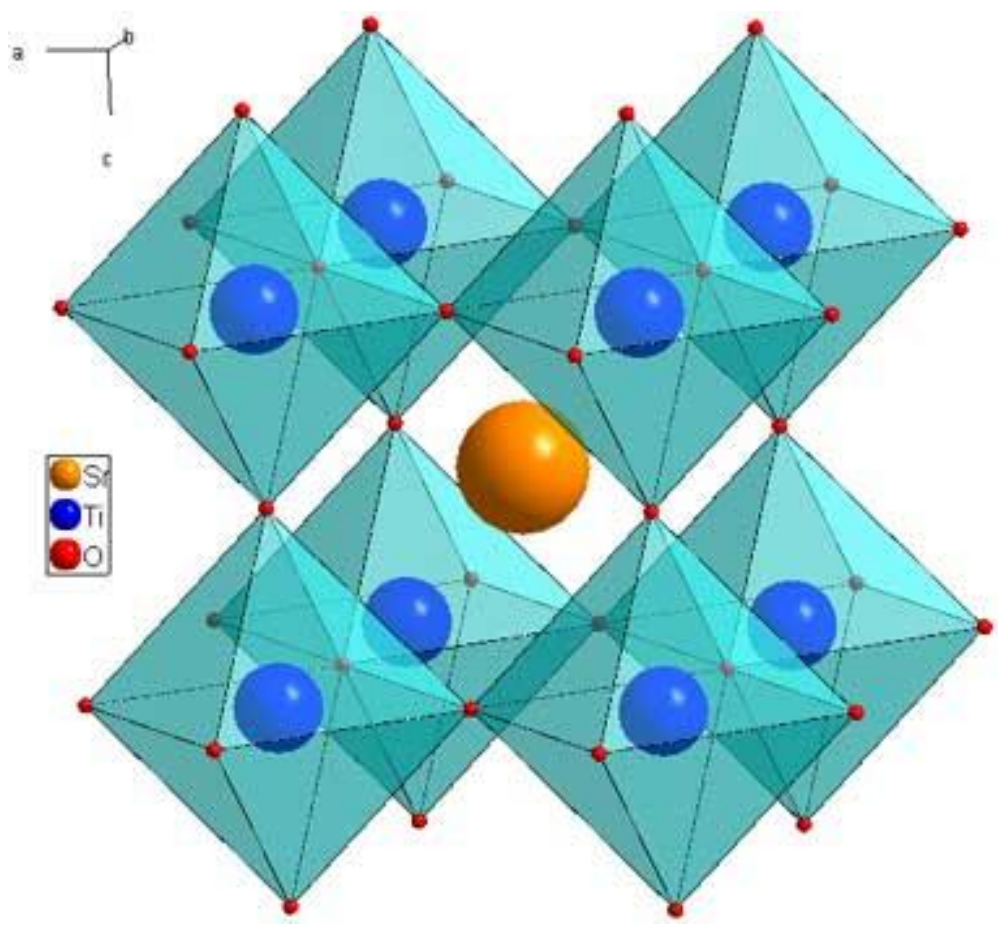

Figure 2.3: Typical cubic perovskite structure [28].

The ideal cubic structure is less common while distortions in the perovskite structure affect their conductive properties. The distortion can be quantified using the Goldschmidt tolerance factor $(\mathrm{t})$ which can be calculated with following formulae [29].

$$
t=\frac{\left(r_{A}+r_{B}\right)}{\sqrt{2}\left(r_{B}+r_{o}\right)}
$$

The ideal cubic perovskite has the tolerance factor equal to unity where displacements of cations and change in structure symmetry by 6-fold coordination site corner tilting cause the tolerance factor to decrease [30]. The oxygen ion vacancies can be introduced by the doping of lower valence materials in the A-site and by reducing the B-site valence which causes a considerable rise in ionic conductivity of the perovskite [31]. 


\subsubsection{Lanthanum Manganite based cathodes}

The lanthanum manganite $\left(\mathrm{LaMnO}_{3}\right)$ is generally doped with strontium ( $\mathrm{Sr}$ ), which improves electrical conductivity of the LSM by enhancing electron hole concentration by oxidizing Mn [24]. Zheng et al. [32] reported a strong dependency of $\mathrm{Sr}$ content and $\mathrm{A} / \mathrm{B}$ ratio of the perovskite on LSM performance. The $\mathrm{A} / \mathrm{B}$ cation ratio greater than unity results in the formation of the $\mathrm{La}_{2} \mathrm{O}_{3}$ minor phase which has tendency to react with YSZ electrolyte forming undesirable lanthanum zirconate, which is considered to be detrimental to the cathode performance. An A/B cation ratio less than unity leads to the formation of $\mathrm{Mn}_{3} \mathrm{O}_{4}$ that enhances the cathode performance; because of this, A-site deficient LSM pervoskites are more opted in SOFC applications. When the $\mathrm{Sr}$ molar content is greater than or equal to $\mathrm{x}=0.3(30 \mathrm{~mol} \%)$, the LSM composition forms a high temperature tetragonal phase which has low electronic conductivity. Mizusaki et al. [33] reported that the electronic conductivity increases with the $\mathrm{Sr}$ dopant concentration in $\mathrm{LSM}\left(\mathrm{La}_{1-\mathrm{X}} \mathrm{Sr}_{\mathrm{X}} \mathrm{MnO}_{3}, 0 \leq \mathrm{x} \leq 0.7\right)$ and the maximum conductivity has been reported to be $\sim 394 \mathrm{~S} / \mathrm{cm}$ at $1000^{\circ} \mathrm{C}$ for $\mathrm{x}=0.5$. Yang et al. [34] investigated the LSM-YSZ composite cathode and reported that the conductivity of the composite is predominantly dependent on concentration of LSM phases. The total conduction in 10 and 20 vol\% LSM-YSZ composites are due to hole and oxygen vacancies, whereas after 20 vol\% (percolation limit) it is completely dominated by LSM phase.

\subsubsection{Lanthanum based Ferro Cobaltite (LSCF) cathodes}

LSCF perovskite cathode is a mixed-ionic and electronic conductor (MIEC), unlike LSM which is limited to only electronic conduction. LSCF being a MIEC offers better catalytic activity and diffusion capabilities over LSM. The oxygen diffusion coefficient of LSCF is observed to be $1 \times 10^{-7} \mathrm{~cm}^{2} / \mathrm{s}$, which much higher than that of LSM $\left(8 \times 10^{-14} \mathrm{~cm}^{2} / \mathrm{s}\right.$ at $\left.800^{\circ} \mathrm{C}\right)$ [35]. The $\mathrm{La}_{0.8} \mathrm{Sr}_{0.2} \mathrm{Co}_{1-\mathrm{y}} \mathrm{Fe}_{\mathrm{y}} \mathrm{O}_{3}$ exhibits a rhombohedral phase for Fe concentrations from $\mathrm{y}=0$ to 0.7 , whereas from $\mathrm{y}=0.8$ to 1 it is orthorhombic at room temperatures [36]. S. Wang et al. [57] confirmed $\mathrm{La}_{1-\mathrm{x}} \mathrm{Sr}_{\mathrm{x}} \mathrm{Co}_{0.2} \mathrm{Fe}_{0.8} \mathrm{O}_{3}$ exhibiting a phase transition from rhombohedral to cubic symmetry between $673 \mathrm{~K}\left(400^{\circ} \mathrm{C}\right)$ and 773 
$\left(500^{\circ} \mathrm{C}\right)$. Tai et al. [37] reported that $\mathrm{Sr}$ content $(\mathrm{x})$ more than 0.6 for $\mathrm{La}_{1-}$ ${ }_{\mathrm{x}} \mathrm{Sr}_{\mathrm{x}} \mathrm{Co}_{0.2} \mathrm{Fe}_{0.8} \mathrm{O}_{3}$ displays secondary phases while rhombohedral being the main phase, which says $x \geq 0.6$ is not preferable in the LSCF composition. A ceria $\left(\mathrm{CeO}_{2}\right)$-based layer is commonly used as a barrier layer between YSZ and LSCF to prevent electrodeelectrolyte reaction. At the usual sintering temperatures of the LSCF $\left(1100-1200^{\circ} \mathrm{C}\right), \mathrm{Sr}$ has a preference to react with the YSZ electrolyte by diffusing through barrier layer forming undesirable strontium zirconate components [38]. Lower sintering temperatures might be a solution, but this affects the microstructure and electrolyte adhesion. Tietz et al. [39] reported that A-site deficient LSCF $\left(\mathrm{La}_{0.58} \mathrm{Sr}_{0.4} \mathrm{Co}_{0.2} \mathrm{Fe}_{0.8} \mathrm{O}_{3-\delta}\right)$ has a higher shrinkage at $1060^{\circ} \mathrm{C}$ than stoichiometric LSCF $\left(\mathrm{La}_{0.6} \mathrm{Sr}_{0.4} \mathrm{Co}_{0.2} \mathrm{Fe}_{0.8} \mathrm{O}_{3-\delta}\right)$, which indicates that a similar microstructure can be obtained by A-site deficient LSCF at lower sintering temperatures by decreasing Sr diffusion issues. This is also supported by Mai et al.'s [40] electrochemical measurements, which displayed that A-site deficient LSCF performed better than stoichiometric LSCF irrespective of sintering temperatures. Petric et al. [41] reported the maximum electronic conductivity of $330 \mathrm{~S} / \mathrm{cm}$ at around $600{ }^{\circ} \mathrm{C}$ for the $\mathrm{La}_{1-}$ ${ }_{\mathrm{x}} \mathrm{Sr}_{\mathrm{x}} \mathrm{Co}_{0.2} \mathrm{Fe}_{0.8}$ with $\mathrm{x}=0.4$. Q. Xu et al. [42] investigated the structural and conductivity properties of $\mathrm{Ln}_{0.6} \mathrm{Sr}_{0.4} \mathrm{Co}_{0.2} \mathrm{Fe}_{0.8} \mathrm{O}_{3-\delta}(\mathrm{Ln}=\mathrm{La}, \mathrm{Pr}, \mathrm{Nd}, \mathrm{Sm})$ by replacing the A-site with smaller lanthanide cations in decreasing order of radii ( $\mathrm{La}, \mathrm{Pr}, \mathrm{Nd}, \mathrm{Sm}$ ). It was shown in this work that the electrical conductivity decreased by replacing $\mathrm{La}^{3+}$ with smaller radii cations, due to a change of the rhombohedral structure to orthorhombic symmetry, and a decrease in the pseudo-cubic lattice constant. Hence, La is better among the available lanthanide cations for cathode material in SOFC applications. Bouwmeester et al. [43] studied the transport coefficients such as surface exchange and diffusion coefficients of oxygen for $\mathrm{La}_{0.6} \mathrm{Sr}_{0.4} \mathrm{Co}_{1-\mathrm{y}} \mathrm{Fe}_{\mathrm{y}} \mathrm{O}_{3-\delta}(\mathrm{y}=0.2,0.5$ and 0.8$)$ using electrochemcial relaxation (ECR) technique measured between $600-800^{\circ} \mathrm{C}$ in oxygen partial pressure between $10^{-4}$ to 1 bar. This work displayed a decrease in both surface exchange and diffusion coefficients of oxygen with decrease in oxygen partial pressure below $10^{-2}$ from which it is derived that better kinetics can be observed in LSCF at atmospheric pressure. 


\subsection{Porous Ceramics}

Materials with a defined and customized porous structure are important in a variety of industrial and research applications. Hence, effective and efficient processes for engineering these porous structures are vital for attaining certain properties for corresponding applications. Porous ceramics have extensive applications because of their broad and attractive electronic, magnetic, and optical properties, as well as their low density and resistance to wear and corrosion. A straightforward method to form homogenous porosity is though sintering ceramic powder at lower temperatures than the corresponding material's actual sintering temperature. This method leads to partial sintering which induces the homogeneous porosity [44]. The pore morphology in a porous ceramic also plays a vital role in applications, so to induce porosity with a controlled pore size distribution and microstructure, which is of special importance in many applications. Studart et al..reviewed some major processing routes to porous ceramics that are explained as follows, and a schematic illustration is shown in Fig 2.4.

a) Replica - In this process the suspension of the desired ceramic is infiltrated into a replica of the required porous structure. The replica here can be either natural or synthetic porous structure that serves as a positive template which will be burned out later leaving the sintered ceramic with desire porous structure of replica. Pores of 10-300 microns with 25 to $95 \%$ porous structures can be obtained. This process also incurs excessive cost and time as multiple steps are involved in conversion of replicas into porous ceramics.

b) Sacrificial Template - This is a negative replica technique where a pore former component or sacrificial material is mixed with ceramic precursor solutions or a ceramic composite. This pore former is removed later, leaving the ceramic precursor with pores as a negative morphology of pore former. The ceramic precursor should possess enough consolidation without collapsing when the pore formers are being extracted. Porous ceramics with pore sizes from 1-700 microns with $20-90 \%$ porosity are generated. Extraction of sacrificial template involves pyrolysis or sublimation which is carried out at very slower rates to avoid cracking of ceramic structure. 
c) Direct Foaming Method: In this method, a gas is incorporated into the ceramic suspension, which will foam the suspension. This gas can be inserted into the solution externally, or a reaction could be initiated within the solution to internally evolve the gas phase. The gas may be trapped within the structure after the solution/suspension gels, and the gas is later released during thermal processing when a continuous porous path is presented through the green structure. Also, the gas may be released and escape the structure during gelation of the solution/suspension, but the void space is retained due to the increased viscosity of the solution/suspension due to polymerization or interparticle networking. The pore structure and porosity is completely dependent upon the gas incorporated and the mobility within the ceramic solution/suspension. Due to travel of the gas and nucleation of gas bubbles inside the suspension, a highly interconnected porosity and wide pore size distribution can be obtained. The size of the pores is predominantly dependent on the wet foam stability. In the wet foam, Ostwald ripening of the gas bubbles can alter the size distribution of the porosity, where several smaller gas bubbles may combine to form one large bubble. The Ostwald ripening and coalescence processes can be controlled with the application of surfactants. This method has an advantage of being simple, fast, and cheap compared to others. Ceramics with 40-97\% porosity with wide pore size distribution with interconnected porosity can be obtained. 


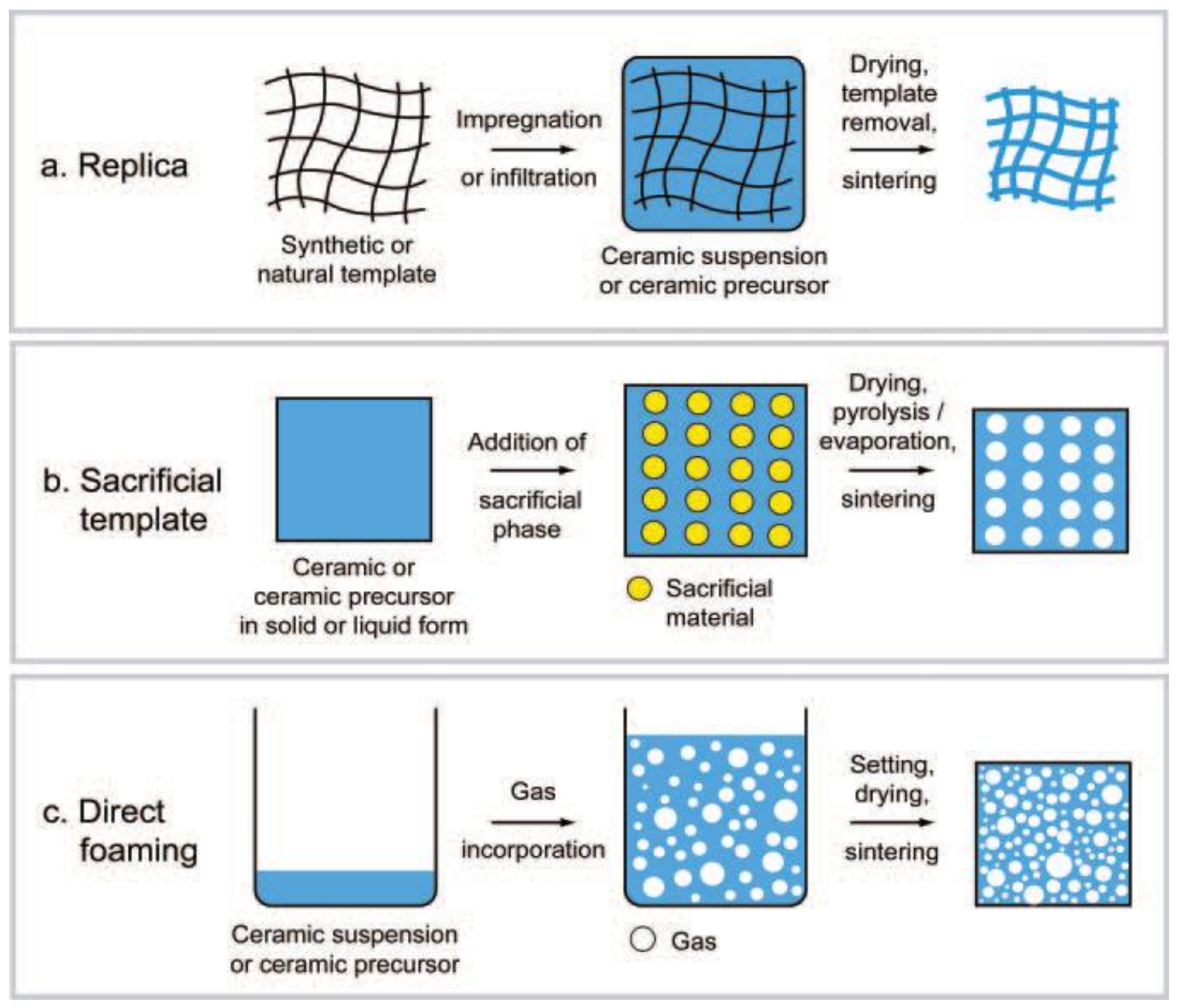

Figure 2.4: Illustration of different techniques for porous ceramics [45].

\subsection{Typical porous structure processing - SOFC cathodes}

It is learned that the cathode microstructure with higher active surface area with interconnected (tortuous) porosity and open pore structure along with the better interfacial contact leads to better adsorption of diffused oxygen along with improved charge transfer.

The porous microstructure of electrodes plays a vital role in mass transport of gas and electrochemical reaction kinetics. The tortuosity, pore size distribution etc. defines the pore architecture that contributes to the performance affecting features such as 
availability of TPB length and active surface area of electrode. Hence, processing a porous electrode through a controlled method is very important. The focus of largely implemented processing routes for porous electrodes is to be easy, consume low processing time and be cost-effective, while producing a structure with $30-50 \%$ porosity level. Large pores can resolve gas diffusion limitations, while at the same time, this may reduce the mechanical strength of the electrode. Large number of fine pores will contribute an increase in active surface area, as well as better strengthen the electrode while it limits the mass diffusion of the gas. Hence, a well-balanced porous structure is of great interest, and will be throughly addressed in this work.

The widely used pore forming technique in SOFC electrodes is using organic pore formers to add porosity. The generally used pore formers are rice starches. The addition of porosity through pore formers is largely dependent on particle sizes of the sacrificial material. It is not just porosity but the shape and orientation of the pores that are very important in determining the electrode performance. Irregularly shaped, interconnected and randomly distributed pores are better suited for enhanced performance of the cathode [58].

The graded porosity across the electrode structure helps to increase interfacial contact (smaller pores) and gas diffusion (larger pores), but the formation of this structure through traditional methods (use of pore formers) is a laborious process requiring multilayer depositions of the cathode. Each layer has to be printed with separate slurries mixed with pore formers of different particle sizes and co-fired [59, 60]. Colombo [61] in his review of conventional and novel processing routes for cellular ceramics reported that use of higher pore generating agents to attain more porosity leads to collapse in cell structure due to large amount of gas evolution during sintering. Also it is reported that slow drying and an optimized pyrolysis process needs to be obtained to avoid cracks in the cell structure due to expansion of pore formers (which generally requires more processing time). The central focus of this work is to use in situ foaming method as an easy and quick tool to produce porous cathodes with interconnected, wide pore size distribution and graded porosity in one direction. 


\subsection{In-Situ foaming in SOFCs}

The adaptability of in situ foaming for ceramic powders were earlier reported by Powell and Evans [46] in their study which yielded a decrease in voids and an increase in relative density of the ceramic foams formed through polyurethane and ceramic suspensions. Wucherer et al..'s [47] work on $\mathrm{BaTiO}_{3}$ foams through direct foaming method explicitly explained that the microstructure of the foam could be directly controlled through a various set of processing parameters. It is reported in their study that lower ceramic loading (20-40 vol\%) will yield better electrical properties due to interconnected cell window with higher porosity (high electron movement), but weaker mechanical properties. The highly porous foams were brittle in nature whereas the higher loaded samples showed an expected higher mechanical strength.

Fig. 2.5 explains the basic polymeric foaming reaction. The standard polymerization reaction is where a di-isocyanate (polymer precursor) along with a hydroxyl group (polyol) yields its respective polymer but the same in presence of water (foaming reagent) generates $\mathrm{CO}_{2}$ gas. This process can be broken into steps for clear understanding as follows:

Step 1- Blowing Reaction: Water molecule reacts with the isocyanate carbon and nitrogen forming carbon-di-oxide and amine group.

Step 2- Gelation Reaction: The polyol reacts with isocyanate forming urea linkage, helping formation of the crosslinking polymer (rigid foam structure). 


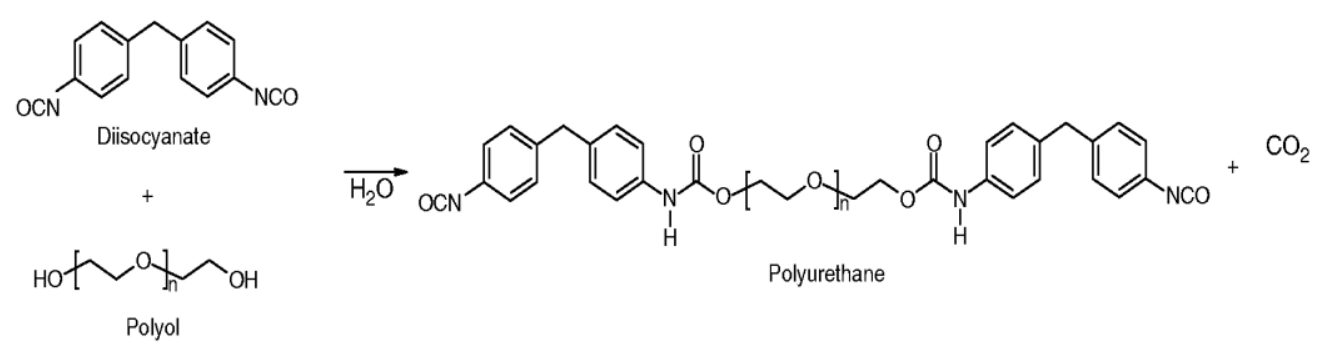

Figure 2.5:Basic polymerization reaction in direct foaming [47].

The in situ foaming process has extensive applications with regards of its simple and quick process, which excludes the slow pyrolysis process. The foamed alumina structures through in situ foaming were used as ceramic insulators in thermal management devices used in SOFC applications due their low thermal conductivity at room temperature and $800^{\circ} \mathrm{C}$ [48]. The use of direct foaming process in the field of solid oxide fuel cells was reported by Rainer et al. [49], where NiO-YSZ substrates were foamed to obtain open cell structure with interconnected porosity for potential anode supports. The demonstration on SOFCs electrolytes has not been reported yet though. The application of in situ foaming for thin film cathodes (or electrodes) is one area which has not shown any previous work and has a great potential for being widely used in electrolyte-supported cells.

\subsection{Nano-catalyst impregnation}

The typical technique used other than changing the composition to enhance the performance of the cathode is the addition of nano-catalyst. The nano-catalyst added to the cathode structure, increases the active region for oxygen reduction by settling over the grain structure across the cathode. This limits the dependency of the active area only at the TPBs. The use of the mixed ionic-electronic conductors and porous cathode architecture will address this issue to an extent, but these nano-catalysts will further enhance the electrocatalytic activity. The wet impregnation/infiltration method is a typical technique used to add nano catalysts to electrodes. Jiang et al. [50] published that the LSM cathode impregnated with gadolinium-doped ceria (GDC) displayed 
polarization resistance of $0.21 \Omega-\mathrm{cm}^{2}\left(700^{\circ} \mathrm{C}\right)$, which is much lower than the pure LSM cathode (70 $\Omega-\mathrm{cm}^{2}$ indicating the effectiveness of the nano catalyst impregnation. The study by Yamahara et al. [51] also reported a higher power density of $270 \mathrm{~mW} / \mathrm{cm}^{2}$ under humidified hydrogen for the SOFC with cobalt impregnated co-fired LSM/SYSZ cathode support which is nearly two times that of the non-impregnated sample.

The cathode backbone for the infiltration can be ionic or electronic, or a mixedconducting material. Although the impregnation of the nano-catalyst into a mixedconducting cathode backbone has been shown to yield less polarization resistance, the stability is of these structures at high-temperature is still a major concern. Ionic conducting backbone reportedly has fewer limitations over the other two and has better stability over a long run of $500 \mathrm{~h}$ [52]. The wet impregnation method, apart from being an alternative method to enhance the cathode (electrode) performance, but suffers with stability limitations at higher temperature operation [53]. The wet impregnation method involves infiltrating the electrode with electro-catalyst precursor solution, which was later added carefully through micropipette or micro-syringe to the electrode. Lack of the open pore structure in traditionally processed electrodes do not allow for the homogeneous infiltration of catalyst. Typical nanomaterial segregation has been identified, where the nanomaterials agglomerate the outer surface instead of diffusing through the thickness to electrode. The interconnected pores yield higher surface area for the electro-catalyst disperses homogeneously improving active surface area for oxygen adsorption and reduction. 


\section{Chapter 3: IN-SITU FOAMING AND EFFECT OF PROCESSING PARAMETERS ON MICROSTRUCTURE}

\subsection{Objective}

The objective of this work is to investigate the effect of in situ foaming processing parameters of LSCF powder on the resultant microstructure. The work in this chapter focused upon bulk foaming of free-form cathode material. The goal is to derive the correlation between the changes in porous microstructure of the cathode with in situ foaming through qualitative analysis of back-scattered scanning electron microscope (SEM) images of the foamed cathode. Later work will investigate engineered cathode microstructures on functional solid oxide fuel cells. The quantitative analysis of pore size, shape, area and distribution was carried out on the microstructures utilizing computational image analysis program (ImageJ by NIH).

\subsection{Experimental}

\subsubsection{LSCF cathode powder synthesis}

The cathode material used in this work was $\left(\mathrm{La}_{0.6} \mathrm{Sr}_{0.4}\right)_{0.98}\left(\mathrm{Co}_{0.2} \mathrm{Fe}_{0.8}\right) \mathrm{O}_{3-\delta}$ (LSCF), a perovskite structured $\left(\mathrm{ABO}_{3}\right)$ mixed ionic and electronic conductor with a $2 \%$ A-site deficiency. This LSCF powder was synthesized through solid-state process. In this process, initially the raw materials of $\mathrm{La}_{2} \mathrm{O}_{3}, \mathrm{SrCO}_{3}, \mathrm{Fe}_{2} \mathrm{O}_{3}$ and $\mathrm{CoCO}_{3}$ are mixed thoroughly in ethanol utilizing zirconia media on a roll mill for $2 \mathrm{~h}$, and then attrition-

milled for $4 \mathrm{~h}$. The slurry is dried and sieved through the $250 \mathrm{mesh}$. The sieved powder is calcined at experimentally determined temperature for $4 \mathrm{~h}$, and then re-attrition milled for $2 \mathrm{~h}$ for finer and uniform grain sized cathode powder. The ethanol is dried from this milled slurry then sieved through 250 mesh again. 
To evaluate the phase evolution during calcination, the X-ray diffraction (XRD) was performed on the calcined powder. The phase development was initially evaluated at three different temperatures $\left(900^{\circ} \mathrm{C}, 1000^{\circ} \mathrm{C}\right.$ and $\left.1100^{\circ} \mathrm{C}\right)$, and these XRD patterns are shown in Fig 3.1. The desired single phase was displayed at $1000^{\circ} \mathrm{C}$, hence the bulk calcination of the powder used in this work was calcined at $1000^{\circ} \mathrm{C}$. The surface area of the LSCF powder calcined at different calcination temperatures were analyzed through Brunauer-Emmett-Teller (BET) technique. This is a surface area analysis technique through physiosorption (physical adsorption) of the liquid nitrogen gas. The surface areas of the LSCF calcined at $900^{\circ} \mathrm{C}, 1000^{\circ} \mathrm{C}$ and $1100^{\circ} \mathrm{C}$ were $3.7289 \mathrm{~m}^{2} / \mathrm{g}, 2.6829 \mathrm{~m}^{2} / \mathrm{g}$ and $1.5788 \mathrm{~m}^{2} / \mathrm{g}$.

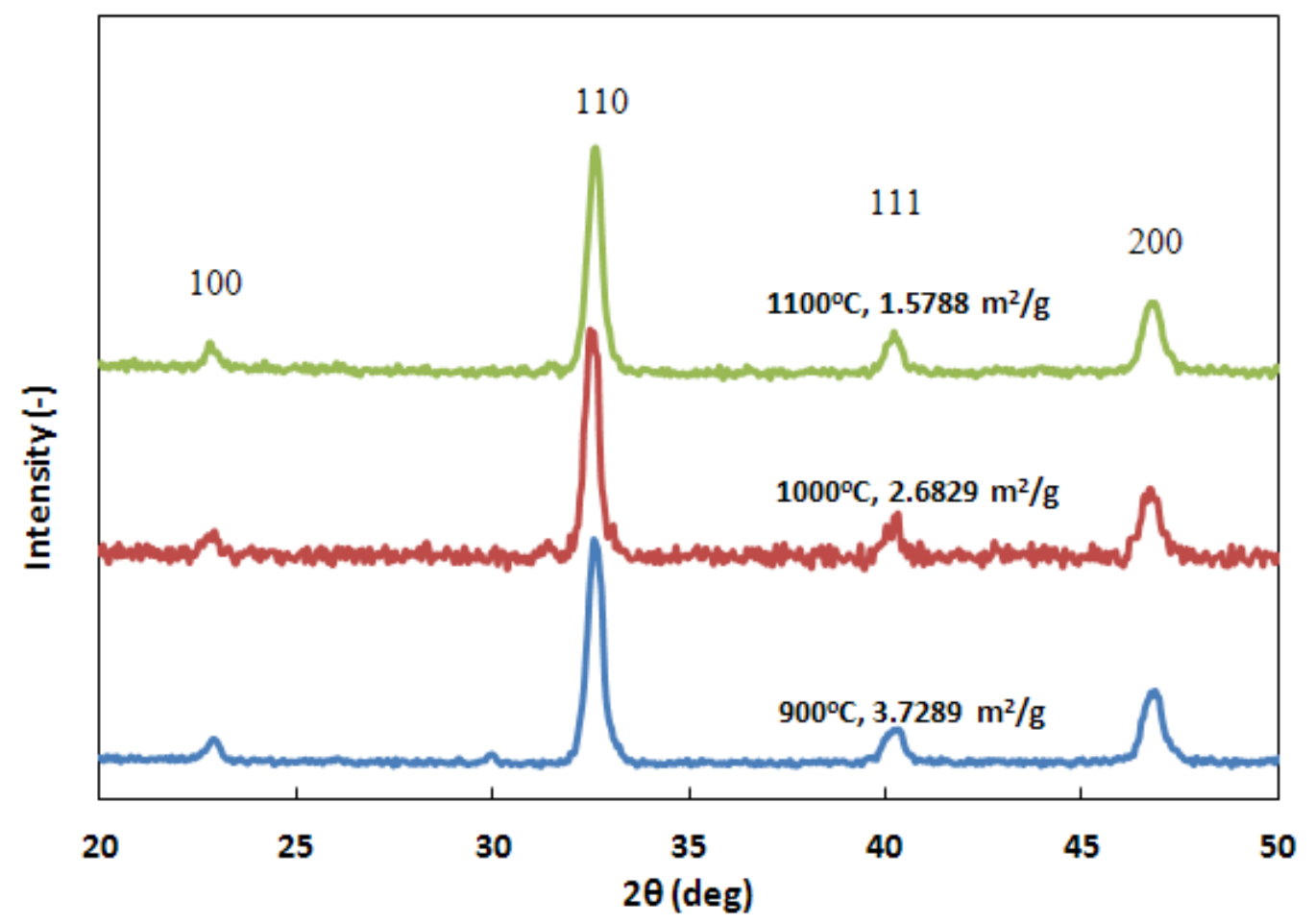

Figure 3.1: XRD pattern of LSCF powder at different calcination temperatures. 


\subsubsection{Conductivity Tests}

Two LSCF powder was synthesized with $2 \%$ A-site deficient $\left[\left(\mathrm{La}_{0.6} \mathrm{Sr}_{0.4}\right)_{0.98}\left(\mathrm{Co}_{0.2} \mathrm{Fe}_{0.8}\right) \mathrm{O}_{3-\delta}\right]$. In the process of determining sintering temperature to fabricate the dense pellets, a sintering study was performed on these samples. The pressed pellets were sintered at different temperatures and then densities were calculated through the Archimedes method. The results of which are shown in Fig. 3.2 where $\%$ theoretical density $(6.448 \mathrm{~g} / \mathrm{cc})$ was graphed with sintering temperatures. The highest theoretical density of $98.3 \%$ was observed in this work at $1450^{\circ} \mathrm{C}$.

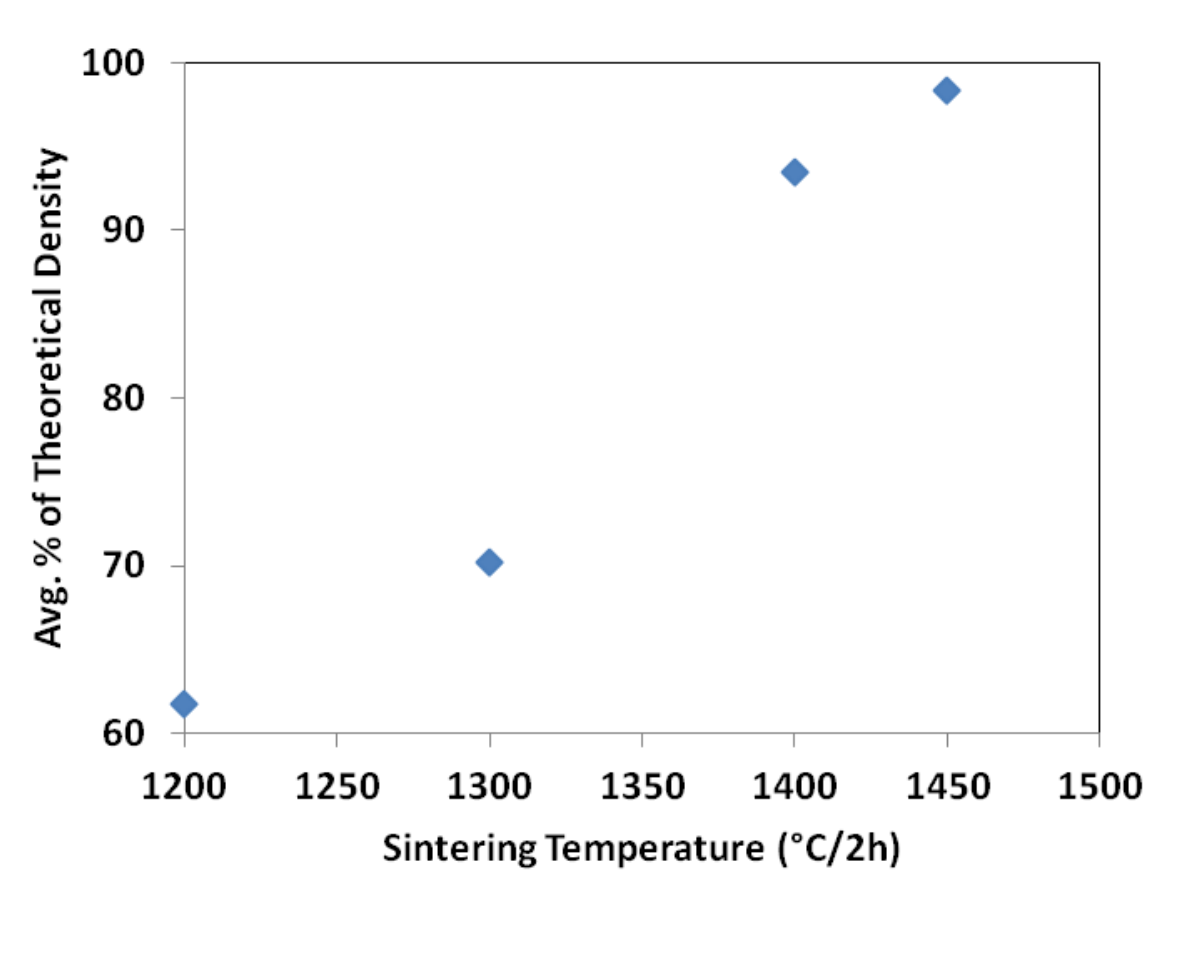

Figure 3.2: Sintering study of the 2\% A-site deficient samples.

The conductivity values were attained on cuboidal shaped pellets sintered at $1450^{\circ} \mathrm{C}$ (from sintering study) through four-point DC conductivity measurement. The 
resistance $(\mathrm{R})$ of the sample of known dimensions at different temperatures was recorded. Later the electrical conductivity of these samples was determined using the following equations:

$$
\begin{aligned}
& \text { Resistivity }(\rho)=\frac{R * A}{l} \\
& \text { Conductivity }(\sigma)=\frac{1}{\rho}
\end{aligned}
$$

The Fig 3.3 exhibits the electronic conductivity versus temperature data for the two compositions. At $800^{\circ} \mathrm{C}$ the $2 \%$ A-site deficient one displayed an electrical conductivity of $250.44 \mathrm{~S} / \mathrm{cm}$. The result is very well in agreement with the Tai et al. 's [73] data of LSCF6428 without A-site deficiency. The A-site non stoichiometry has no significant influence on electronic conductivity but improve the ionic conductivity and oxygen catalytic properties for reduction [74]. Hence, A-site deficient cathode serves as better cathode for electrochemical performance which is confirmed by Templeton et al. [75] by reporting $10 \%$ higher cell power densities in their study of the effect of A-site non-stoichiometry on the LSCF cathodes.

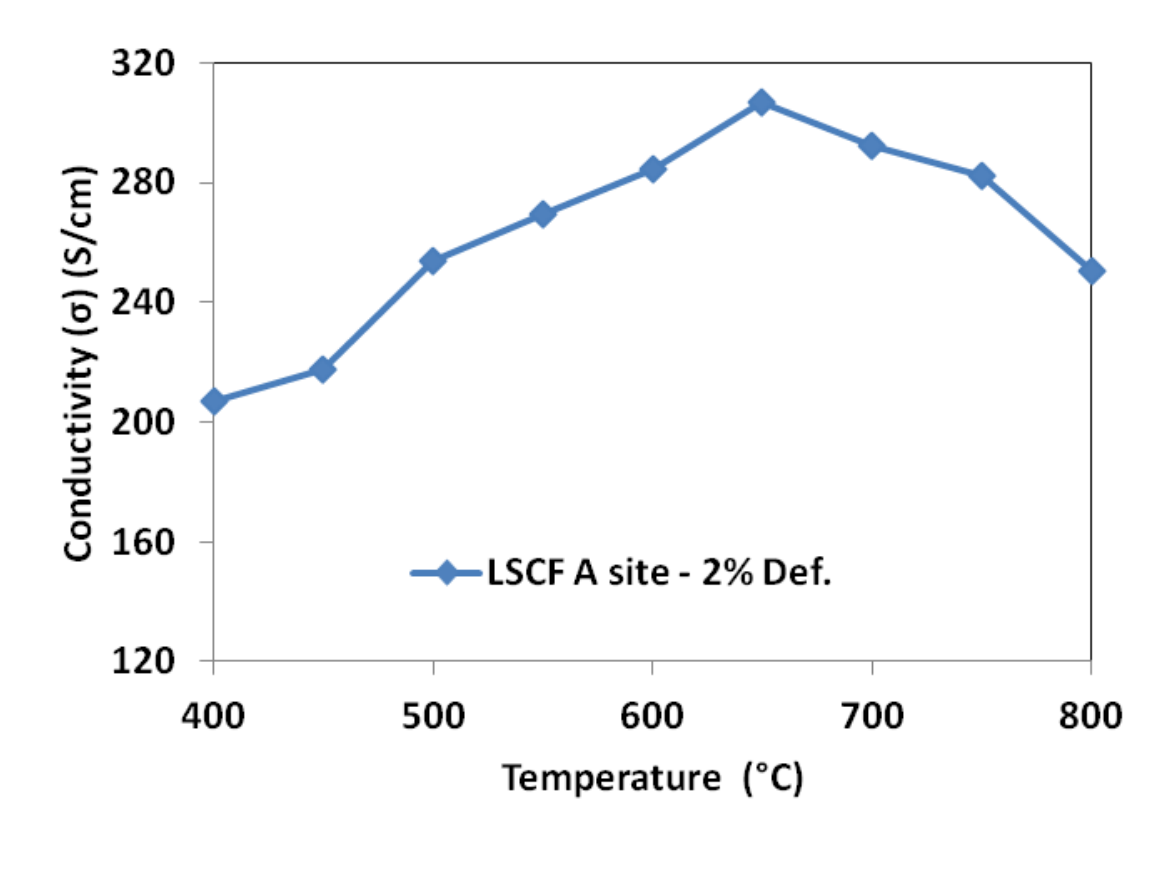

Figure 3.3: Electronic conductivity versus isothermal temperature data for Asite deficient cathodes. 


\subsubsection{In-situ foaming process}

The direct foaming method is an adaptable process that yields microstructures with a broad distribution of pore size and tortuosity. In situ foaming (direct foaming) method occurs through the evolution of gas bubbles within the ceramic suspension through a chemical reaction. The porosity retained within the ceramic after sintering, leaves voids where gas bubbles were evolved. The porosity in this process is highly dependent on gas composition, gas bubble coalesce and gas migration through the solution/suspension. Here, the foaming process is a result of a polyurethane polymerization reaction that leads to foam the ceramic suspension by yielding carbon dioxide gas. The basic polyurethane polymerization reaction needs an isocyanate to react with a hydroxyl (polyol). In this work, the foaming suspension contains both cathode particles dispersed in ink vehicle (Johnson Matthey 62/3 medium) and a polymer precursor solution. Later, the foaming suspension is exposed to a reagent for a polymerization reaction to occur. The exposure of the polymer precursor mixture to the reagent (water) produces carbon dioxide $\left(\mathrm{CO}_{2}\right)$ gas as byproduct of the reaction. The carbon dioxide produced during the polymerization reaction leads to the foaming of the cathode. The direct foaming process of a cathode printing ink leads to low density and high surface area microstructure, which are considered to be desirable features to improve performance of SOFC cathodes.

In this work, a polyurethane (PU) precursor consisting of polymethylene polyphenyl isocyanate and polyol (PEG 200) with polyoxyethylene sorbitan monooleate (Tween 80) as a Si-free surfactant was used for the polymerization reaction [62]. The foamed structure through in situ foaming is destabilized due to Ostwald ripening and coalescence; in order to stabilize the structure, the Tween 80 surfactant is required [63]. The polymer precursors are mixed in a predetermined volumetric ratio. This PU precursor mixture is then mixed with the cathode material in an appropriate volume ratio pertaining to desired solids loading. 
The $\mathrm{CO}_{2}$ gas evolved during polymerization reaction is dispersed and trapped through the solution/suspension. Internal pressure may result from accumulating trapped gas which results in the reorganization of the micron-size cathode powder within the suspension. Reorganization of the cathode microstructure requires a highly viscous suspension to encapsulate the evolving gas during polymerization. A solution composed of terpineol and ethyl cellulose (viscosity around 10,000 $\mathrm{cP}$ at room temperature) has been used as viscous particulate carrier. Ethyl cellulose increases the suspension's viscosity and also acts as binder to increase the green strength of the final polymer/ceramic composite. Foamed sample is then burned out at a rate of $1^{\circ} \mathrm{C} / \mathrm{min}$ to $600^{\circ} \mathrm{C}$, which burns off all the organics associated with cathode suspension and foaming process. The burned out structure is then sintered at a rate of $3^{\circ} \mathrm{C} / \mathrm{min}$ to the determined sintering temperature and held for $2 \mathrm{~h}$ to produce the desired porous architecture.

\subsubsection{Computational Image Analysis -Image J}

Sample preparation for back-scattered SEM imaging: The application of the image analysis technique by taking back-scattered SEM images at different locations of a 2D polished surface and averaging the microstructural characteristics to derive data was reported by Lanzini et al. [64]. The sintered cathode structures were mounted in a low viscosity epoxy set (Allied Hitech Products, Inc) mixture with a weight ratio of 100:12 (epoxy resin: hardener) for the cross section imaging. The low viscosity epoxy was used, so that it completely impregnates the pores of the cathode microstructure, which helps in clearly differentiating the pore and solid during the analysis. Air bubbles from this slurry were removed by vacuum, and the sample is later cured at room temperature for $8 \mathrm{~h}$. The epoxy mounts were then polished down to 1200 grit along with a $1 \mu \mathrm{m}$ diamond paste polishing. This helps in yielding a two-dimensional planarized view of the foamed cathode's porous architecture through SEM imaging. The SEM images were obtained using JEOL 7600 (West Virginia) scanning electron microscope with back-scattered imaging technique. Back-scattered imaging allows for easier differentiation between 
pores and solids. Every sample was imaged at 3 different locations under 3 different magnifications $(500 \times, 1000 \times$ and $2000 \times)$. Every data point represented for any pore character is an average of 9 data points (images) per sample. The SEM back scattered image clearly distinguishes the pore and solid with dark and brighter spots within the image, respectively.

ImageJ is computational image analysis software offered by the National Institute of Health (NIH), which is an open-ware package that allows customized java plug-ins to be drafted according to individual requirements. Line-intercept method can be used for quantitative analysis of microstructure through image analysis which yields information related grain/pore size and volume fractions [65, 66]. Impoco [67] has reported a software plug in for ImageJ to analyze the SEM images of intricate and interconnected porous microstructures of cheese which is more relevant to the microstructures of foamed cathode that are obtained through in-situ foaming. This method has been adopted and modified to analyze foamed cathode microstructures in this work.

SEM images acquired were processed and analyzed using ImageJ. The edges of the pore boundaries were smoothened while contrast levels were adjusted; later images were binarized using ImageJ program as shown in Fig 3.4. The change in contrast and brightness along with binarizing the image helps clearly distinguish between different phases in the micrograph and defining clear grain boundaries [65, 68]. The porous structure characterization was performed using the computational statistics obtained through ImageJ, which yields mean pore area, form factors, porosity, pore aspect ratio, pore maximum and minimum diameter. Deriving a unique shape factor that defines pore character of each sample was crucial in this process. The unique shape factor allows for a quick and easy method to determine the microstructural characteristics of the porous microstructure, especially for the given foamed microstructures that display such a wide pore size distribution and highly interconnected porosity. The circularity factor of pores was considered to be the shape factor that defined pore character and distribution with microstructure. This factor was significant because it yielded clear characteristics about the shape, formation and packing of pores. This factor is defined as ratio of mean 
perimeter of the pore to mean Ferret's diameter, which was obtained using Image $\mathbf{J}$ analysis as shown in Fig 3.5 (i.e. pore circle when this factor is equal to $\pi$ ). As the factor approaches the value of $\pi$, the pore shape is more circular. The farther the value of the factor is from $\pi$ the closer the pore is to an elongated or interconnected channel. The analysis allows deriving correlation between processing parameters of in situ foaming and porous microstructure (pore formation behavior).

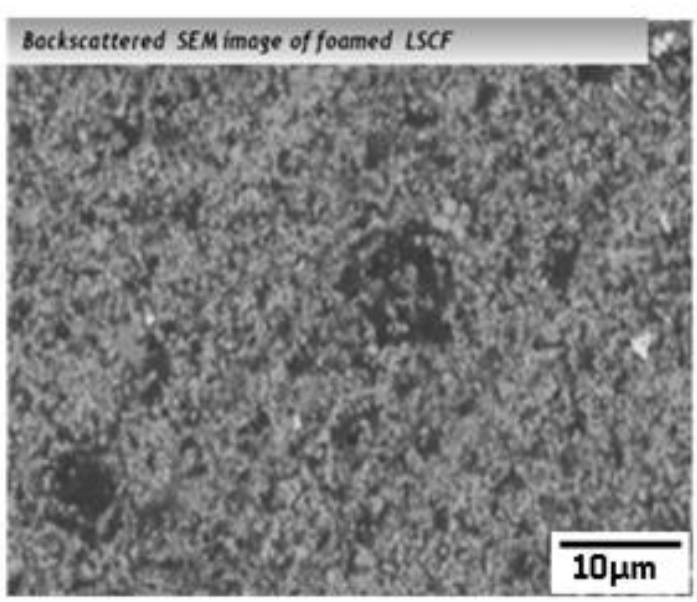

Porosity by Archimedes

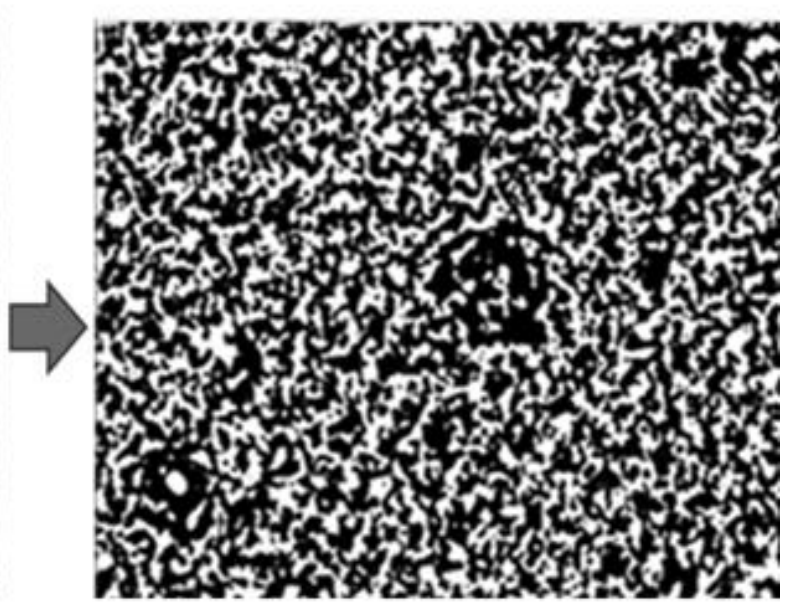

Porosity by Image $\mathrm{J}$ analysis $59.5 \%$

Figure 3.4: (Left) Back scattered SEM image of a low viscous epoxy infiltrated foamed LSCF sample which is binarized (right) for phase differentiation computational image analysis. 


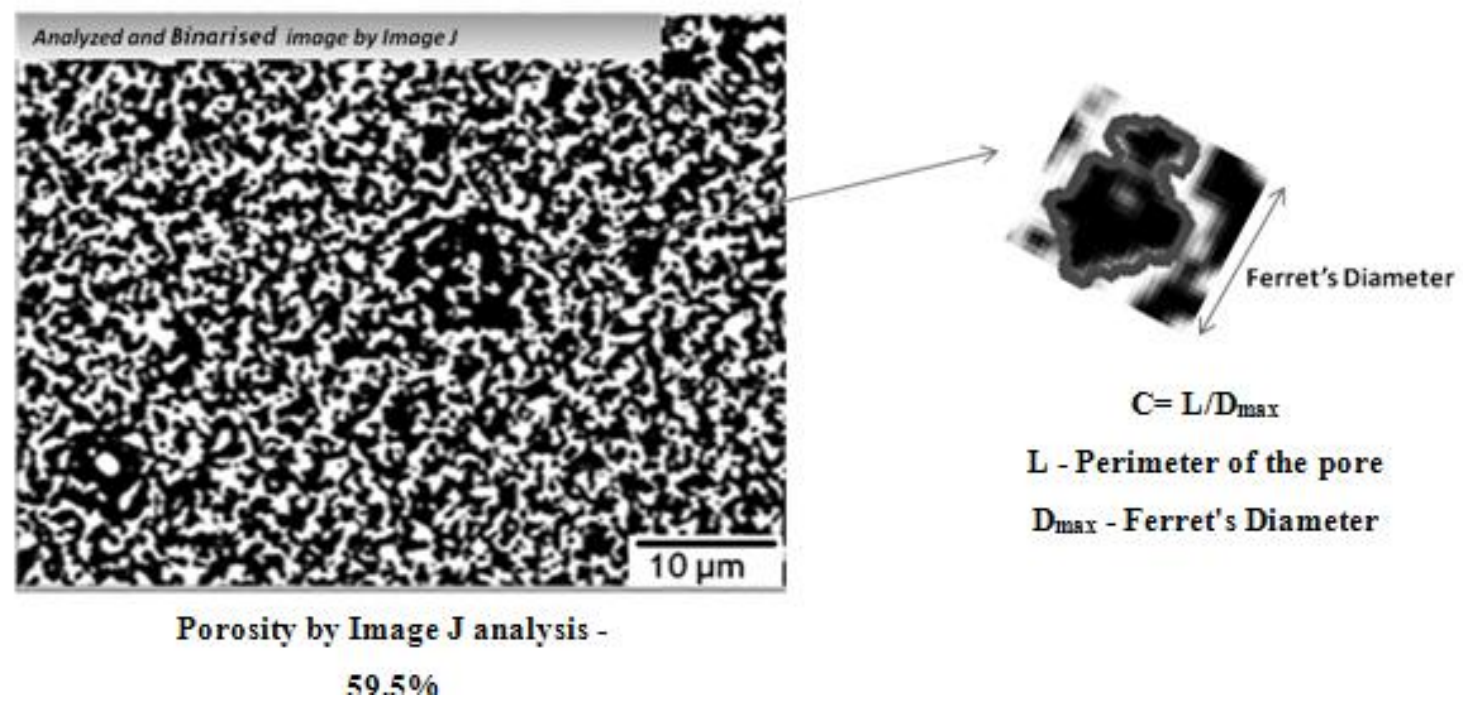

Figure 3.5: Method for deriving circularity factor from image analysis.

\subsection{Results and Discussion}

\subsubsection{Ceramic Suspension and its Constituents}

The first step in in situ foaming the cathode is to determine the favorable constituents for the ceramic suspension with the desired properties. Rheological study of the LSCF powder at different volume ratios was performed to understand and determine the viscosity behavior of the ceramic suspension. The initial set of experiments was performed to study the viscous behavior of the powder in different solvents with no polymer precursors. A mixture of ethyl cellulose and terpineol is a standard ink vehicle that is used to prepare inks for screen-printing in SOFC applications. Hence, the ink vehicle was considered for suspending the LSCF powder for this study. LSCF was mixed thoroughly (by sonication) with ink vehicle at different volume ratios of LSCF to ink vehicle such as 1:5 (45-55 wt\%), 1:4 (50-50 wt\%) and 2:5 (62.5-37.5 wt\%). Fig 3.6 displays the rheology data of the above-mentioned three compositions, where viscosity is mapped against the shear rate. It shows that all the compositions exhibits shear thinning behavior (decrease in viscosity with the increase in shear rate) of the ceramic suspension. 
This means it behaves as viscoelastic fluid which starts flow after a certain stress threshold is crossed and is considered to be most suited for screen-printing the cathode ink, because of the lower de-agglomeration process [69]. It is learned from this study (Fig 3.6) that a volume ratio of $2: 5$ for LSCF to ink vehicle has viscosity in the region of $8,000-10,000 \mathrm{cP}$ at and around shear rate of $10 \mathrm{~s}^{-1}$, which is similar to that of commercially available LSCF inks with solids loading of $62-72 \mathrm{wt} \%$ and viscosity range of $15,000-40,000 \mathrm{cP}$ at a shear rate of $10 \mathrm{~s}^{-1}$ [70]. The much higher viscosities were observed at all shear rates for higher solids loading composition (2:5) than others in this study. This high viscosity levels at lower shear rates (near $\mathrm{O} \mathrm{s}^{-1}$ ) helps in encapsulating the evolved $\mathrm{CO}_{2}$ gas resisting the buoyancy effect, which allows bubbles to escape during the in situ foaming process to retain the foamed structure from collapsing due to the escape of gas.

Further experiments were performed to investigate the effect of adding polymer precursors on rheology while same volume ratio solid to solvent is maintained constant. This set of experiments were performed with different solvents such as terpineol and ethanol along with ink vehicle and also with polymer precursors - polmethylene polyphenyl isocyanate, PEG 200 and surfactant (stoichiometric vol. ratio of 8:4:1) for insitu foaming. This study was performed to investigate the effect of solvents and the polymer precursors on the rheology of the ceramic suspension. Fig 3.7 shows the viscosity behavior against the shear rate for different solvents. It indicates that although the entire solvents displayed shear thinning behavior, the ceramic suspension with ethanol displayed a very low viscosity of $400-500 \mathrm{cP}$ at a shear rate of $10 \mathrm{~s}^{-1}$, which is lower than the typical level for screen-printing. Both the ceramic suspensions with ink vehicle and terpineol have higher viscosities at lower shear rates while terpineol exhibited a very high viscosity of $25,300 \mathrm{cP}$ at $2 \mathrm{~s}^{-1}$ when compared to ink vehicle $(11,900 \mathrm{cP}$ at 2 $\left.\mathrm{s}^{-1}\right)$.

The above study indicates that a volume ratio of 2:5 (LSCF:solvent) with both ink vehicle and terpineol displayed higher viscosities at shear rates of 1.5-2.0 $\mathrm{s}^{-1}$, which helps to have enough surface tension balancing the bubble pressure and buoyancy effect to encapsulate evolved $\mathrm{CO}_{2}$ during in situ foaming. The porous microstructure was characterized with these solvents to investigate their effect with 70-30 (vol\%) of LSCF- 
polymer precursor mix and a 8:4:1 polymer precursor composition sintered at $1150^{\circ} \mathrm{C} / 2$ h. From Table 3.1, it is evident that sample foamed with vehicle as the solvent has a higher mean pore area of $15.93 \mu \mathrm{m}^{2}$ than one with terpineol $10.91 \mu^{2}$ under similar porosity (58-60\%). The distribution of the pore area with a change in solvent (Fig 3.8) clearly shows that broader pore size distribution was obtained using the vehicle carrier, whereas the use of the terpineol carrier solution resulted in a higher pore concentration with lower average pore area. A higher mean pore area aids in increase of TPBs and surface area for oxygen to reduce in cathode. Hence, for the rest of experiments in this work, vehicle was used as the solvent.

Isocyanates are used as the monomers to produce polymers and are a vital component in polyurethane foaming reaction. The isocyanate monomers generally used in polyurethane foaming reaction are 4,4'-diphenylmethane diisocyanate (MDI), toluene diisocyanate (TDI), hexamethylene diisocyanate (HDI), and isophorone diisocyanate (IPDI). These isocyanates can be further grouped into aromatic (TDI, MDI) and aliphatic (IDPI) isocyanates. The effect of using aromatic and aliphatic isocyanates as polymer precursor on the microstructure was examined by bulk foaming of the cathode with vehicle as solvent while varying the isocyanate and rest of the parameters were maintained constant as in previous experiment. The results from image analysis were shown in Fig 3.9 where pore size distribution mapped with both the isocyanates. It indicates the broader pore size distribution was generated with polymethylene polyphenylisocyanate (aromatic), whereas with the isophorone diisocyante monomer (aliphatic), a higher concentration of pores was observed with a lower mean pore area. Polymethylene polyphenylisocyanate (aromatic) displayed a mean pore area of 15.93 $\mu \mathrm{m}^{2}$, which is twice the value when compared to that of Isophorone diisocyante (aliphatic), $6.21 \mu^{2}$ obtained using with the same porosity level (54-58\%). The microstructure generated from the aromatic isocyanate has pores with a mean circularity factor of 6.90 , which is $40 \%$ more tortuous than those generated by aliphatic as show in Table 3.1. The reason for sample with aliphatic isocyanate lacking ability to generate broad pore size distribution and large pore areas can be explained as the aliphatic monomers are slower in reaction rate with polyol when compared to other [76]. The reaction rate of water (foaming reagent) with isocyanate to generate $\mathrm{CO}_{2}$ gas during 
foaming is faster than that of isocyanate with polyol forming cross linking polymers. Hence, slower reaction rate of the isocyanate to polyol yields in a delay of crosslinking urea which weakens the foam structure by lacking enough strength in the cell windows to balance the bubble pressure. This leads to collapse of pore structure by the lack of rigid foam [77]. Hence, the polymethylene polyphenylisocyanate was continued as polymer precursor in the further investigations of this work.

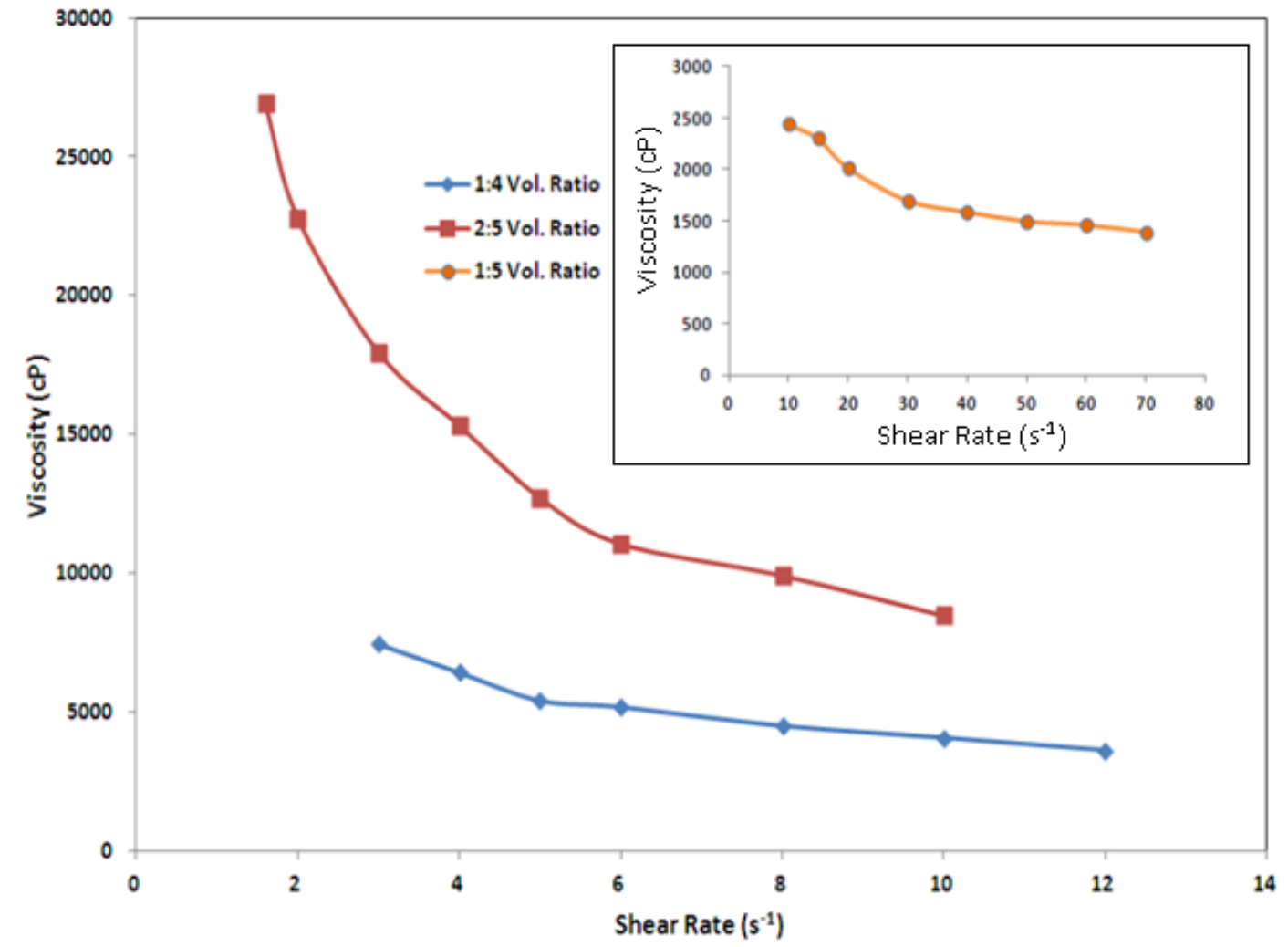

Figure 3.6: Viscosity Measurements LSCF with ink vehicle with different volume ratios of solid to solvent. 


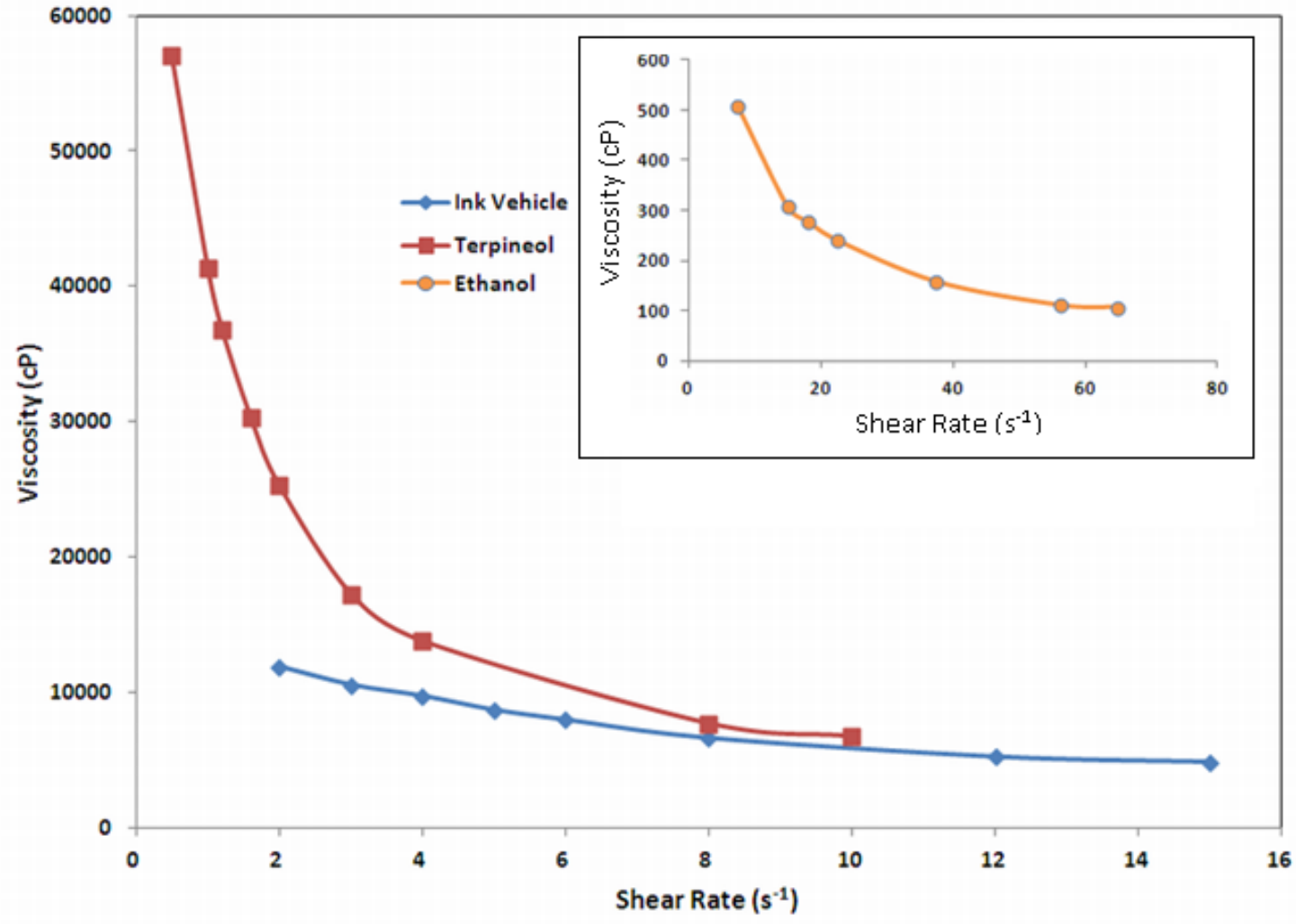

Figure 3.7: Viscosity measurements of LSCF + polymer precursors' mixture (8:4:1) with different solvents at constant 2:5 vol ratio of solids to solvent, terpineol and ink sintered at $1150^{\circ} \mathrm{C}$. 
Table 3.1: Effect of solvents and polymer precursor on the microstructure of bulk foamed cathode with 70\% solids loading away from the YSZ substrate, sintered at $1150^{\circ} \mathrm{C}$.

\begin{tabular}{|c|c|c|c|c|c|}
\hline $\begin{array}{c}\text { Porosity } \\
\text { (\%) }\end{array}$ & $\begin{array}{c}\text { Mean-Ferret's } \\
\text { Diameter } \\
(\mu \mathrm{m})\end{array}$ & $\begin{array}{c}\text { Mean- } \\
\text { Perimeter } \\
(\mu \mathrm{m})\end{array}$ & $\begin{array}{c}\text { Mean- } \\
\text { Circularity } \\
\text { Factor }\end{array}$ & $\begin{array}{c}\text { Mean-Pore } \\
\text { Area } \\
\left(\mu \mathrm{m}^{2}\right)\end{array}$ & Solvent \\
\hline 58.53 & 2.55 & 17.79 & 6.90 & 15.93 & Vehicle \\
\hline 60.95 & 2.43 & 16.16 & 6.61 & 10.51 & Terpineol \\
\hline & & & & & $\begin{array}{c}\text { Polymer } \\
\text { Precursor }\end{array}$ \\
\hline 58.53 & 2.55 & 17.79 & 6.90 & 15.93 & $\begin{array}{c}\text { Polymethylene } \\
\text { polyphenyl } \\
\text { isocyanate }\end{array}$ \\
\hline 54.38 & 2.73 & 13.55 & 4.90 & 6.21 & $\begin{array}{l}\text { Isophorone } \\
\text { Diisocyanate }\end{array}$ \\
\hline
\end{tabular}




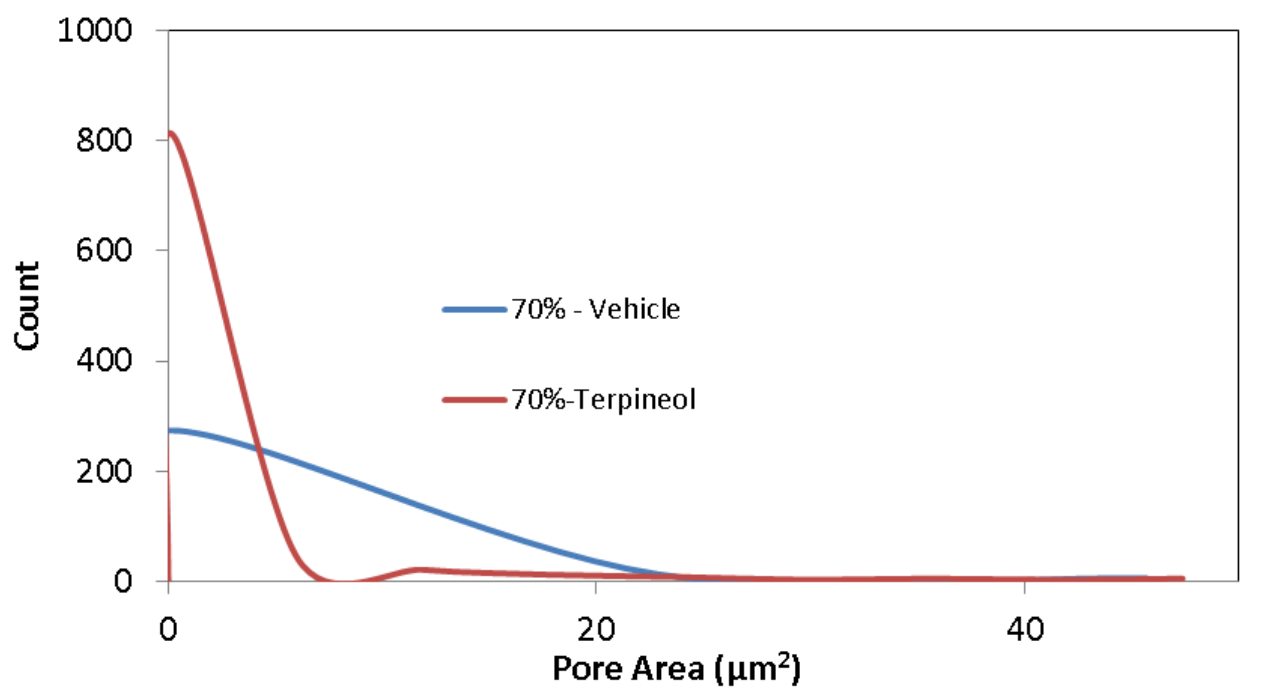

Figure 3.8: Pore count versus pore area of sample foamed with $70 \%$ solids loading with change inn solvents sintered at $1150^{\circ} \mathrm{C}$.

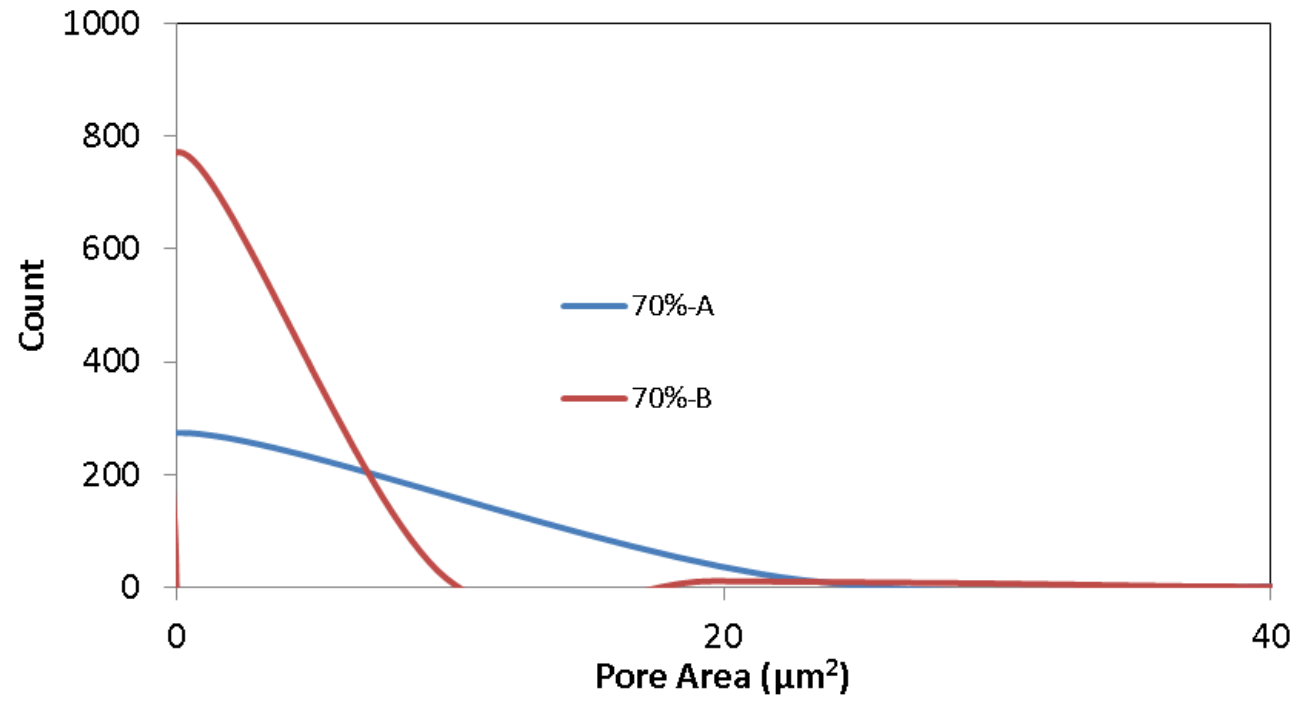

Figure 3.9: Pore count versus pore area of sample foamed with $70 \%$ solids loading with change in isocyantaes sintered at $1150^{\circ} \mathrm{C}$. (A-polymethylene polyphenylisocyanate; B-Isophorone diisocyanate) 


\subsubsection{Effect of sintering temperature}

The experiments in this section were designed to understand the effect of sintering temperature on the foamed microstructure. The LSCF cathode was foamed with two different solids loading 50 and $70 \%$ and at constant stoichiochiometric polymer precursor composition (isocyanate: polyol: surfactant-8:4:1) for both loadings. These samples at each solids loading were later sintered at 4 different temperatures $(1050,1150,1250$ and $1350^{\circ} \mathrm{C}$ ) for $2 \mathrm{~h}$. The image analysis study as shown in Fig. 3.10 indicates porosity, circularity factor and mean pore area plotted against sintering temperature. It shows that at both the solids loading (50\% and 70\%) a significant fall in porosity and mean pore area was observed at $1250^{\circ} \mathrm{C}$ and $1350^{\circ} \mathrm{C}$ when compared to those of at $1050^{\circ} \mathrm{C}$ and $1150^{\circ} \mathrm{C}$. The large fall in circularity factor to values close to $\pi$ (3.14) indicates the collapse of interconnected porosity leaving discrete circular pores. The sintering is considered to be consolidation of the loose particles with decrease in surface area and porosity due small particle agglomeration and structure shrinkage leading to densification increasing the density of the sample by heating [78]. It is evident from results that the densification of the foamed cathode through grain growth was intensified resulting in collapsing of pore architecture at and above $1250^{\circ} \mathrm{C}$, which is undesirable. Later through tape tests where a scotch tape will be peeled against the electrode to check its adhesion to electrolyte, it is learned that cathodes foamed over electrolyte had better adhesion at $1150^{\circ} \mathrm{C}$ than $1050^{\circ} \mathrm{C}$. Hence $1150^{\circ} \mathrm{C}$ is considered to be a suitable sintering temperature and was continued for the rest of the work. The back scattered SEM image of bulk foamed cathode with $70 \%$ solids loading and 8:4:1 polymer precursor's composition away from the YSZ substrate, sintered at $1350^{\circ} \mathrm{C}$ (top) and $1050^{\circ} \mathrm{C}$ (bottom) are shown in Fig 3.11, where densification cathode can be clearly seen at $1350^{\circ} \mathrm{C}$. 


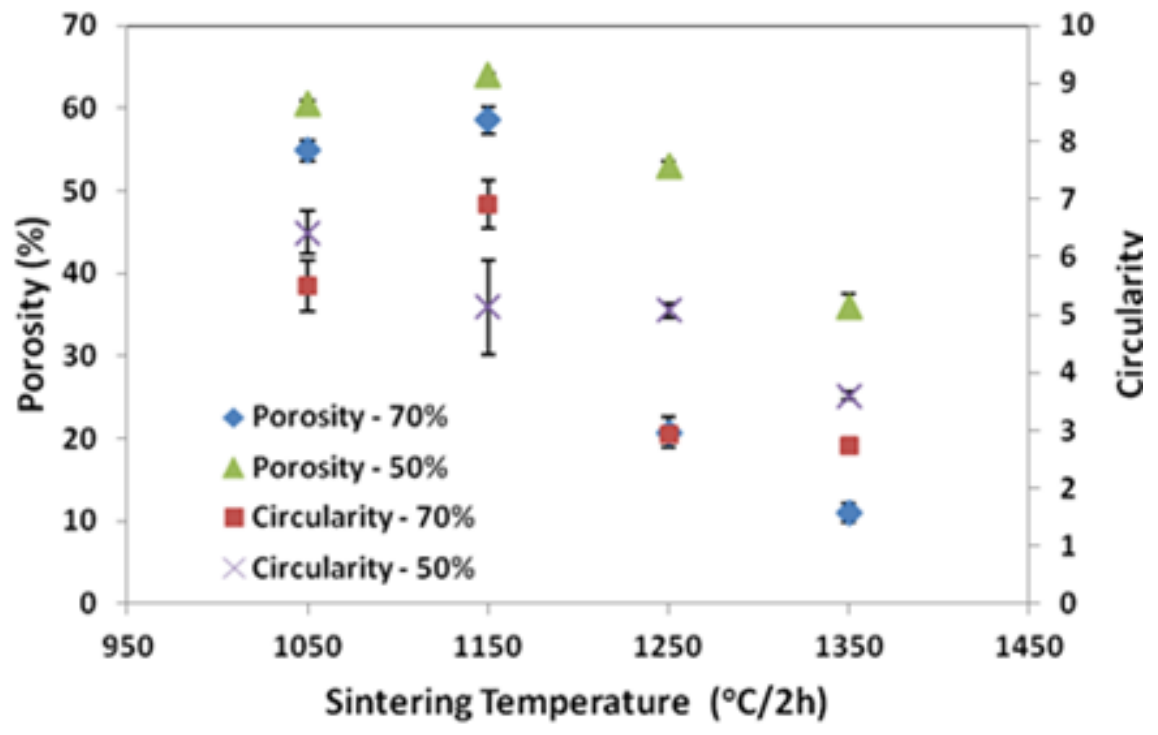

(a)

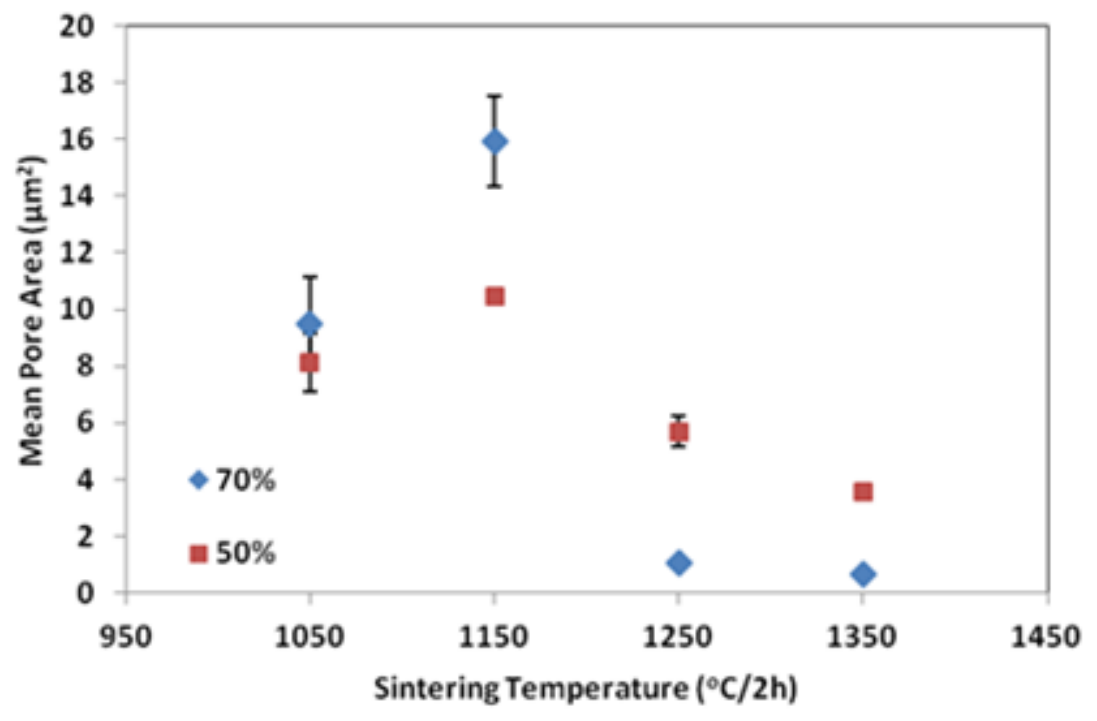

(b)

Figure 3.10: Effect of sintering temperature on microstructure of bulk foamed cathode with 70\% and 50\% solids loading at 8:4:1 polymer precursor's composition away from the YSZ substrate. 


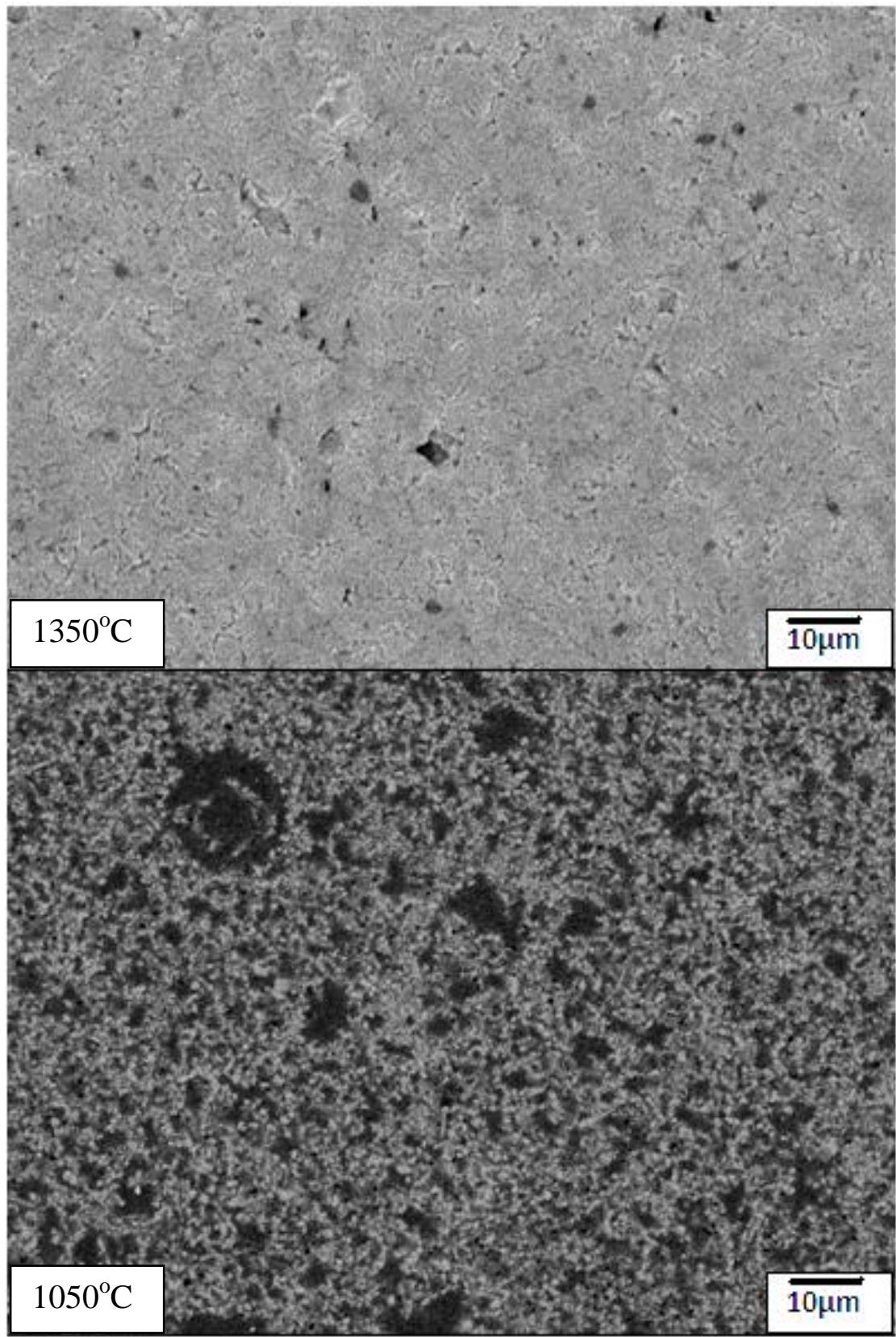

Figure 3.11: Back scattered SEM image of bulk foamed cathode with 70\% solids loading and 8:4:1 polymer precursor's composition away from the YSZ substrate, sintered at $1350^{\circ} \mathrm{C}$ and $1050^{\circ} \mathrm{C}$. 


\subsubsection{Effect of Solids Loading}

Experiments in this section were designated to understand the effect of solids loading on microstructure of in situ foamed cathode. A set of experiments with different solids loading of cathode such as 30,50, 55, 60, 65 and 70 vol\% in ceramic-polymer precursor suspension while retaining the stoichiometric precursor composition of volumetric ratio-8:4:1 (precursor: PEG 200: surfactant) as constant for all solids loadings. As shown in Fig.3.12, the pores were more elongated or interconnected channels with circularity factor farther from $\pi$ (3.14) at higher solids loading though there was no significant change observed between $60 \%$ and $70 \%$ solids loading. Pores being interconnected and elongated channels in a cathode are desirable features for SOFC applications as it aids gas diffusion through it. A decrease in porosity is observed with an increase in solids loading, which is a natural phenomenon; hence they behaved, as they should. There is almost a $10 \%$ decrease in porosity observed from 50 vol\% to 70 vol\% solids loading with $58 \%$ porosity at 70 vol\% solids loading. The low porosity (63\%) at $30 \%$ solids loading can be explained as the lower solids loading has loosely packed solid material and which accounts for not balancing the bubble pressure and buoyancy effect. This leads to the coalescence of higher amount of bubbles and due to the higher bubble pressure that overcomes the surface tension leading to escape of $\mathrm{CO}_{2}$ gas evolved leaving pores to collapse before setting. There was an appreciable rise of the mean pore area observed with increase in solids loading, with the highest mean pore area around $16 \mu \mathrm{m}^{2}$ observed at 70 vol\% solids loading. This phenomena of increasing trend of mean pore area with solids loading is related to the low buoyancy effect in densely packed material yielding heterogeneous bubble nucleation and bubble coalescence at locally concentrated pores instead of homogeneously dispersed throughout the fine porosity. This is again a desirable feature in cathode structure for SOFC applications, by increasing the mean pore area the overall surface area for gas-solid interaction increases. Due to the mixedconduction capabilities of LSCF, this increased solid-gas area provides a larger total area for the oxygen reduction reaction, thus potentially increasing the kinetics for oxygen incorporation into the electrolyte phase. 
The distribution of pore area was wider at 70 vol\% solids loading when compared to others. The trend of the pore size distribution with solids loading is shown in Fig 3.14. Hence, the $70 \mathrm{vol} \%$ solids loading is considered to be an optimum solids loading and higher solids loading yields better mechanical strength. The back scattered SEM images of bulk foamed cathode with 8:4:1 polymer precursor's composition at $30 \mathrm{vol} \%$ and 70 vol\% solids loading sintered at $1150^{\circ} \mathrm{C}$ are shown in Fig 3.13. 


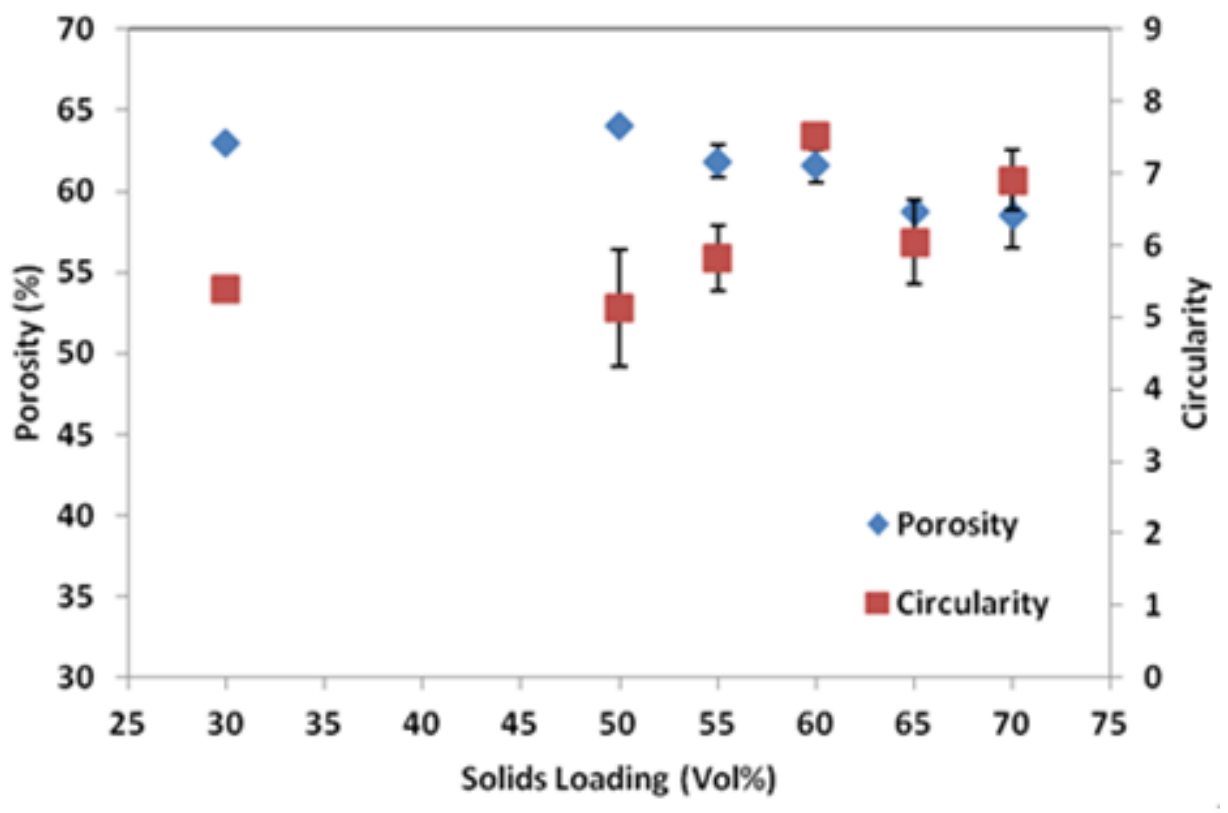

(a)

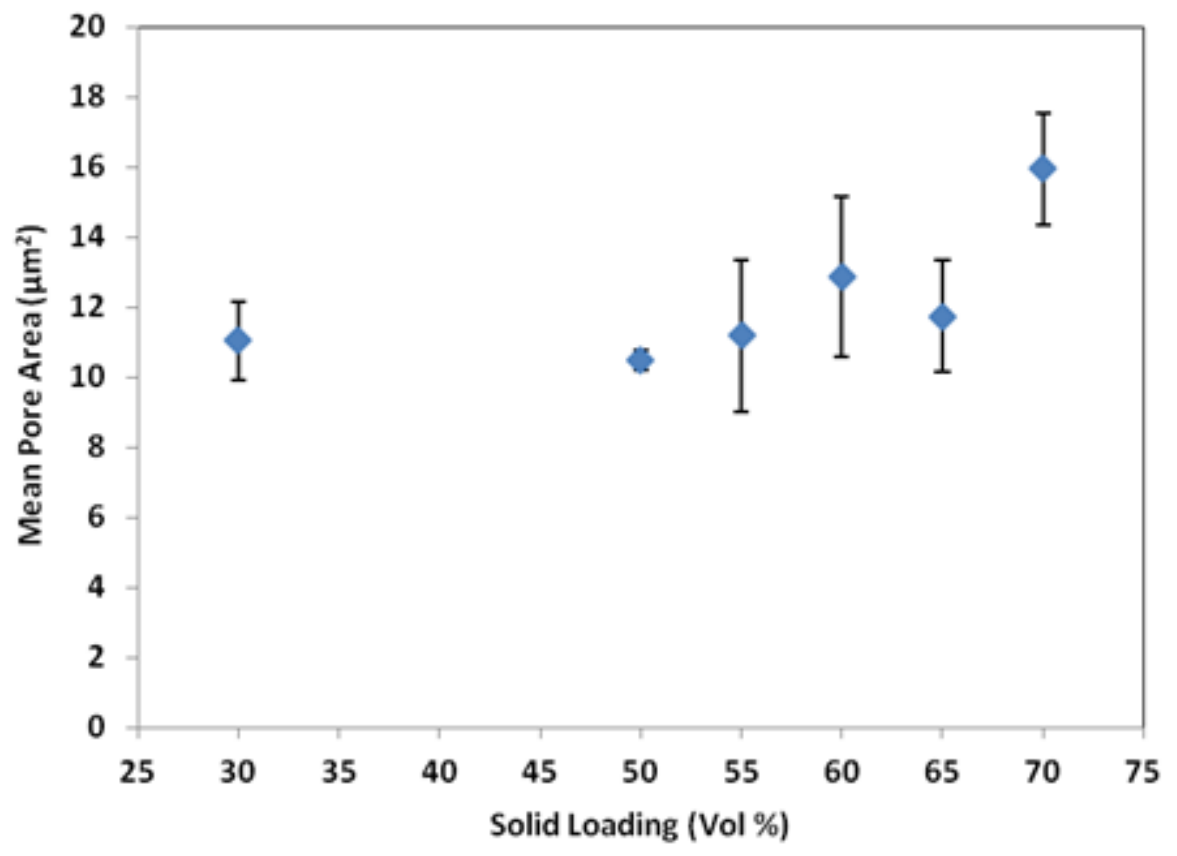

(b)

Figure 3.12: Effect of solids loading on microstructure of bulk foamed cathode with 8:4:1 polymer precursor's composition - away from the YSZ substrate, sintered at $1150^{\circ} \mathrm{C}$. 


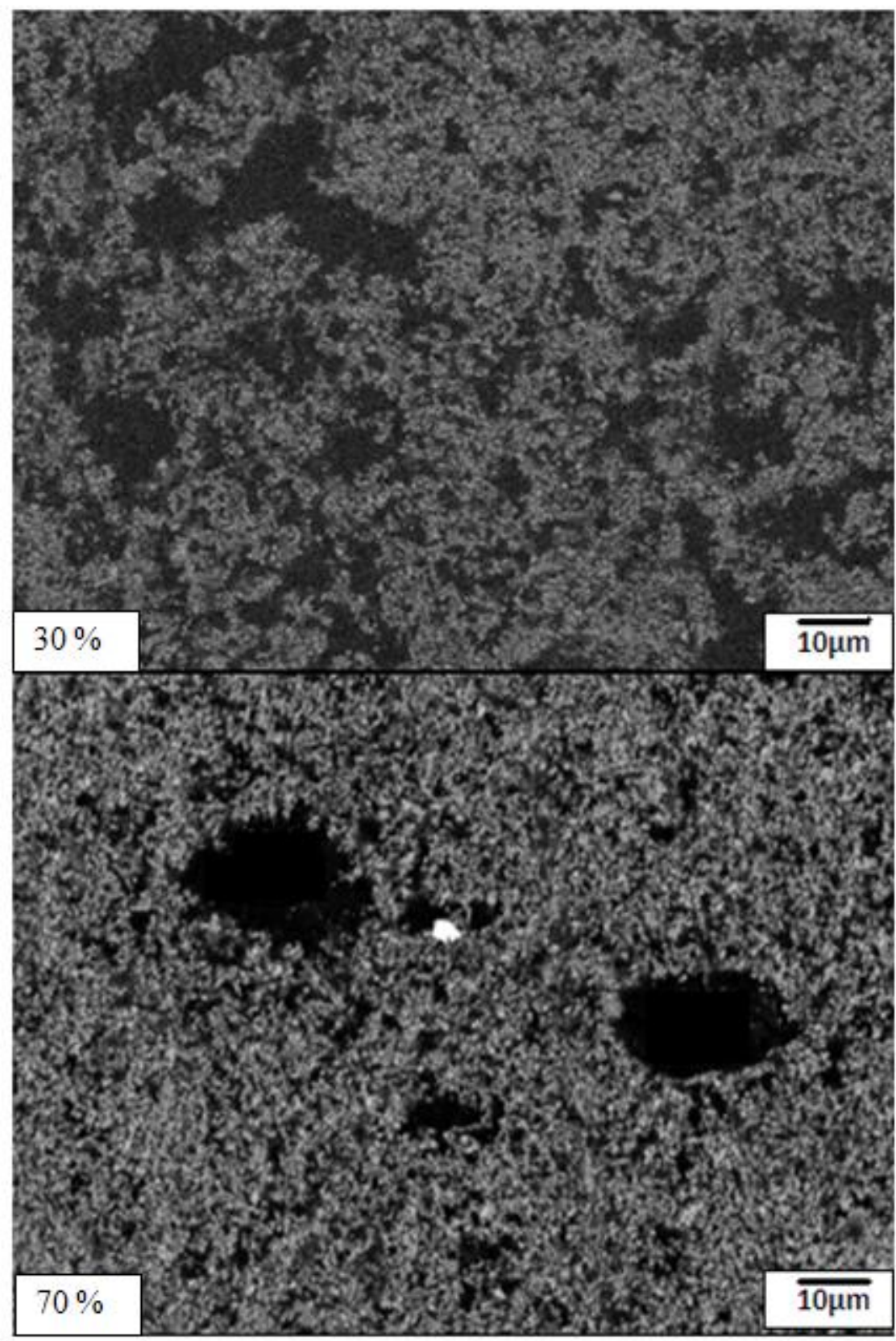

Figure 3.13:Back scattered SEM image of bulk foamed cathode with 8:4:1 polymer precursor's composition at 30 vol\% and 70 vol\% solids loading, sintered at $1150^{\circ} \mathrm{C}$. 


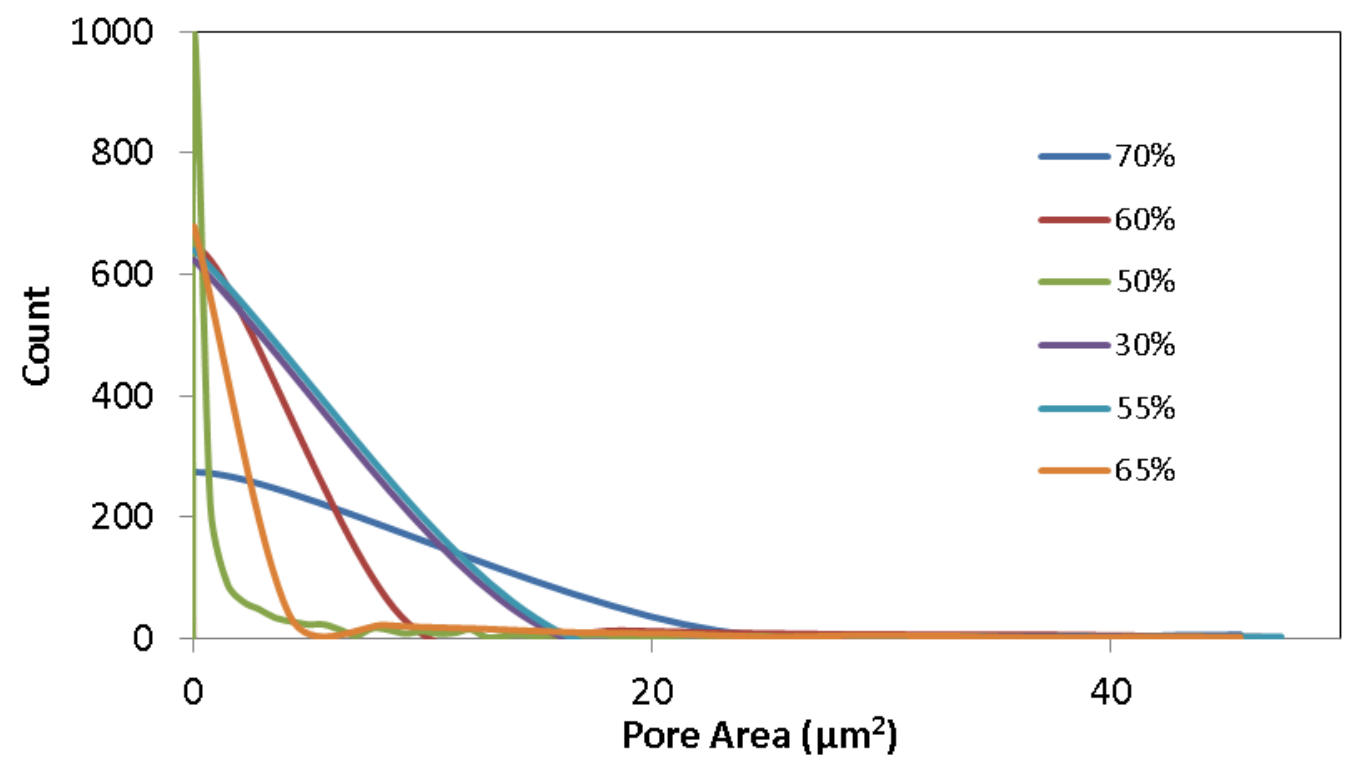

Figure 3.14: Pore area distribution (pore count versus pore area) as a function of solids loading for LSCF samples foamed with 8:4:1 composition and sintered at $1150^{\circ} \mathrm{C}$.

\subsubsection{Effect of polymer precursor composition}

This study focused on varying polymer precursor compositions in the ceramicpolymer suspension while retaining the solids loading constant at $70 \mathrm{vol} \%$. The following precursor compositions were evaluated at the isocyanate: polyol: surfactant volume ratios of 12:2:1, 8:6:2, 9:4:1, 8:4:1 and 8:4:2. The stoichiometric ratio of isocyante to hydroxyl group to initiate the polymerization reaction during polyurethane foaming is $2: 1$; the changes in microstructure were investigated by moving away from stoichiometric ratio to account for diffusional and mixedness variations within the solution. In the bulk foamed samples (as shown in Fig 3.15), there was no significant change in porosity observed with changes in precursor, which was just within a 5\% (porosity) window. The trend was expected since the samples were foamed at constant solids loading. Interestingly, the pores formed with the stoichiometric polymer precursor composition of 8:4:1 (precursor: PEG 200: surfactant) exhibited more interconnected porosity as the circularity factor was found to be $\sim 50 \%$ farther from $\pi$ than the other compositions tested. Also, the higher 
mean pore area was observed at the same composition compared to others tested for similar porosity levels. Nearly three times higher mean pore area was attained with stoichiometric composition compared to non-stoichiometric compositions 12:2:1, 8:6:2 and 9:4:1. The other stoichiometric composition (8:4:2) with higher surfactant concentration yielded pores with circularity factor $(\sim 4)$ much closer to $\pi$ which means that the pores were more circular and less interconnected. Also, these samples demonstrate a $50 \%$ reduction in the mean pore area over those foamed with the $8: 4: 1$. In general, the surfactant decreases the surface tension, stabilizes the foam by strengthening cell windows and prevents coalescence or drainage effect while helping in nucleation of bubbles [71]. An increase in the surfactant concentration will increase in the hydraulic resistance in cell windows which results in over stabilization and shrinkage of the foam [72]. The gas bubble propagating pressure cannot counter against cell windows due higher surface tension yielding small and less tortuous (more circular) pores, this explanation supports the results from this work. The distribution of pore area was wider at 70 vol\% solids loading with the 8:4:1 stoichiometric polymer precursor concentration when compared to others. Back-scattered SEM image of bulk foamed cathode with 8:4:1, 8:4:2 and 12:2:1 polymer precursor composition at $70 \mathrm{vol} \%$ solids loading, sintered at $1150^{\circ} \mathrm{C}$ is shown Fig 3.16.The trend of the pore size distribution with solids loading is shown in Fig 3.17. Hence, the 8:4:1 ratio is considered the optimal polymer precursor composition for the production of the tortuous and higher mean pore area pores. There appears to be very little kinetic restrictions on the reaction which would benefit from the higher polyol additions. The 8:4:2 and 9:4:1 ratios are also examined in further work for electrochemical testing for a strong statistical conclusion. 

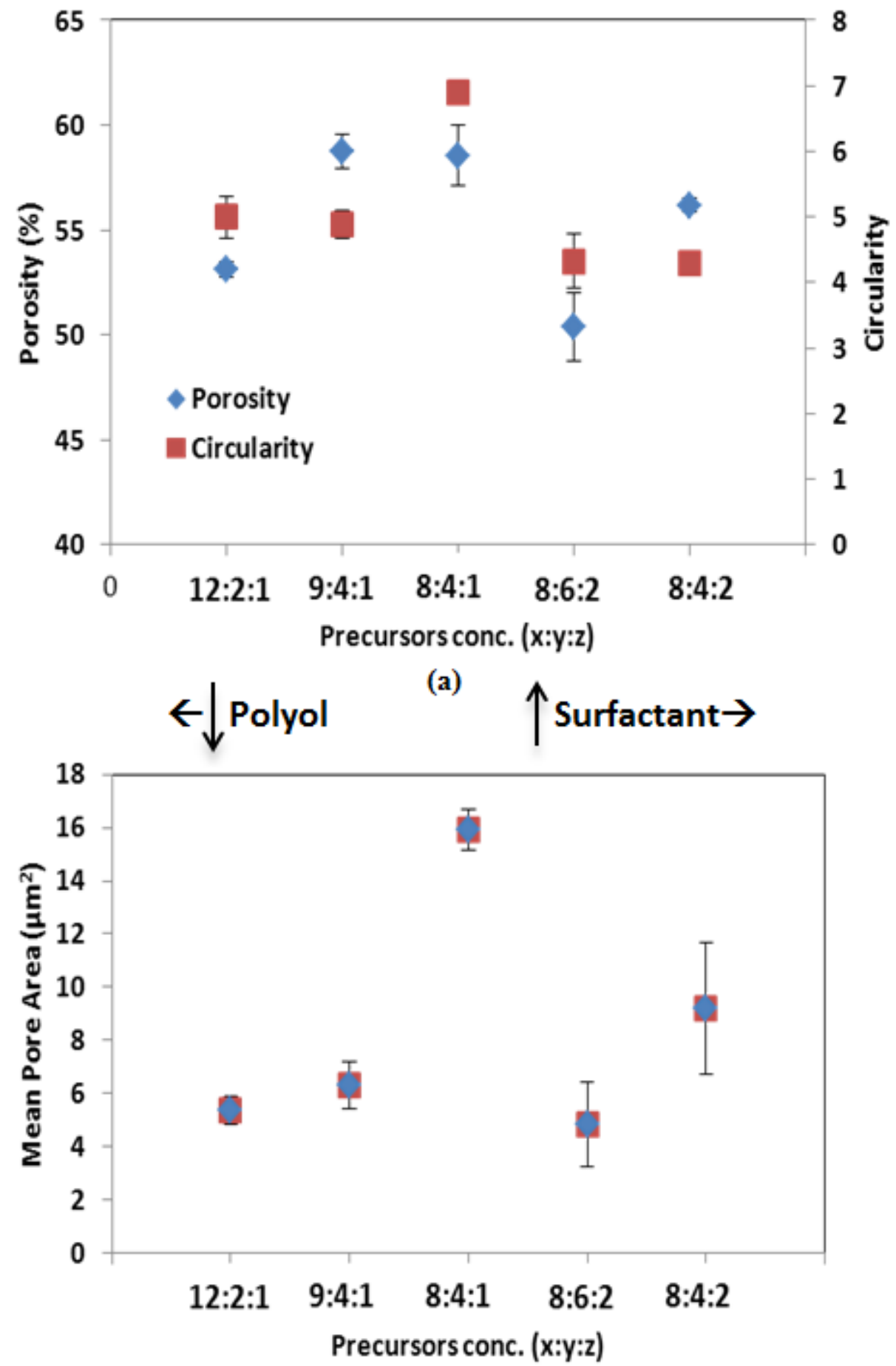

(b)

Figure 3.15: Effect of polymer precursor's composition on microstructure of bulk foamed cathode with $70 \%$ solids loading away from the YSZ substrate, sintered at $1150^{\circ} \mathrm{C}$. 


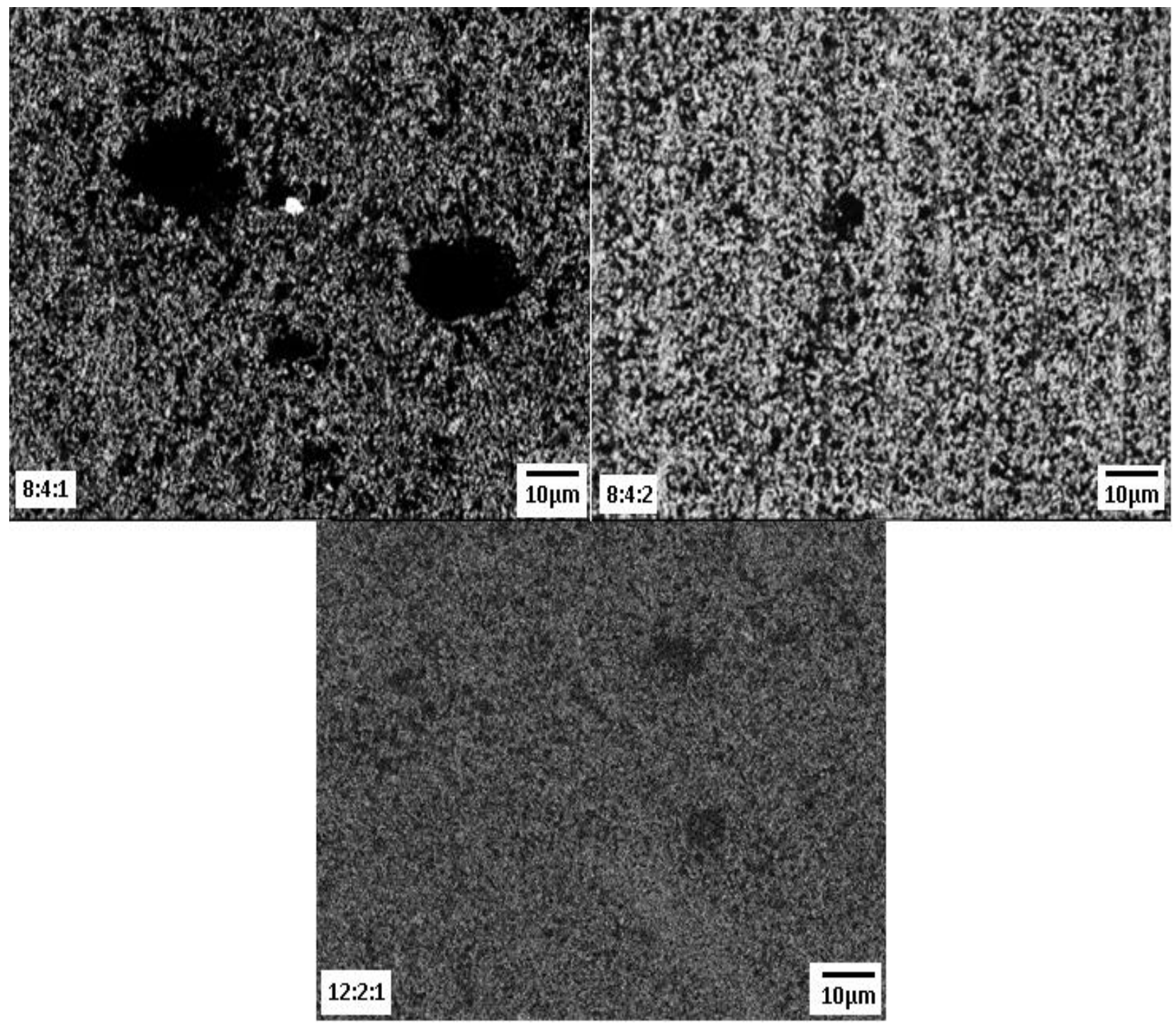

Figure 3.16: Back scattered SEM image of bulk foamed cathode with 8:4:1, 8:4:2 and 12:2:1 polymer precursor's composition at 70 vol\% solids loading, sintered at $1150^{\circ} \mathrm{C}$. 


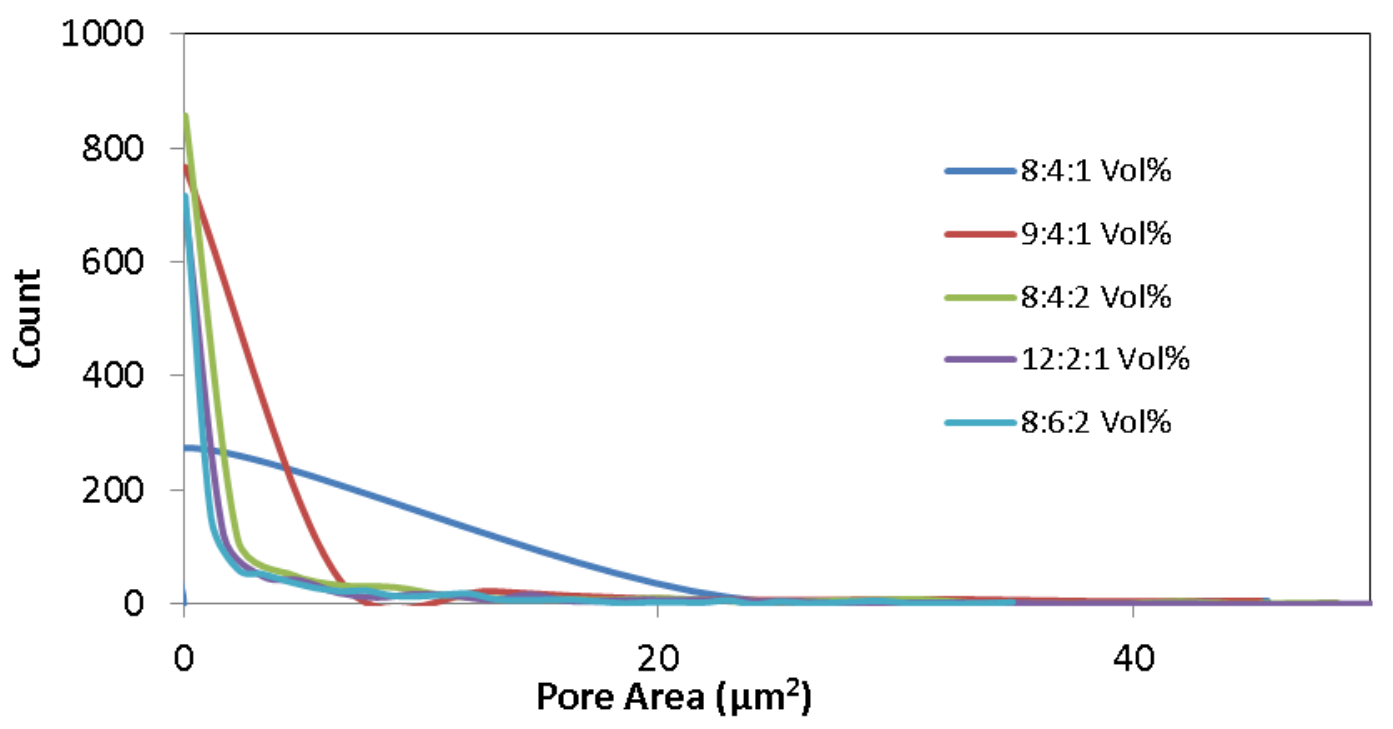

Figure 3.17:Pore area distribution (pore count versus pore area) as a function of precursor composition for LSCF samples foamed with 70\% solids loading and sintered at $1150^{\circ} \mathrm{C}$.

\subsection{Conclusion}

The use of the direct foaming process, which is relatively unconventional pore forming strategy in the SOFC applications, was investigated on the cathode (LSCF). The governing forces of the bubble nucleation and growth were controlled through the change in the materials and composition of the processing parameters to obtain improved cathode microstructure. The quantitative analysis of the microstructural properties with change in the processing parameters was completed through $2 \mathrm{D}$ image analysis to derive the correlation between them. This study indicated that the foamed cathode processed with the ink vehicle as solvent at a 2:5 (solids: solvent) volume ratio, using an aromatic isocyanate as polymer precursor and $70 \mathrm{vol} \%$ solids loading. A foamed cathode using a near stoichiometric foaming precursor composition (8:4:1) that was sintered at $1150^{\circ} \mathrm{C}$ yielded an improved microstructural properties. The improved microstructural properties of the cathode with the open-pore architecture and interconnected porosity with the enhanced active surface area was obtained by governing the reaction mechanism of bubble nucleation and growth. 


\section{Chapter 4: IN SITU FOAMING OF CATHODE OVER ELECTROLYTE AND ELECTROCHEMICAL TESTING}

\subsection{Objective}

The objective of this part of work is to investigate the transfer of the in situ foaming process demonstrated in Chapter 3 to a process for depositing thick film LSCF cathodes over the YSZ substrate required for SOFC operation. The primary focus is to verify whether the microstructure of the foamed cathode over the YSZ electrolyte is displaying a similar microstructure development as that of the bulk foaming with the change in precursor composition. The developmental work is aligned with the establishment of a process for foaming thick film cathodes over the substrate and investigating the electrochemical performance compared to that of traditional LSCF cathodes. This work used symmetrical cathode cells and full solid oxide fuel (button) cells to characterize the processing-microstructure-performance relationship. The electrochemical testing was performed to investigate the effectiveness of the foamed microstructure over the baseline (unfoamed) microstructure.

\subsection{Experimental}

\subsubsection{Cathode symmetrical cells fabrication}

Baseline: The electrolytes for electrolyte-supported symmetrical half-cell testing were fabricated by tape casting and lamination of $8 \mathrm{~mol} \% \mathrm{Y}_{2} \mathrm{O}_{3}-\mathrm{ZrO}_{2}$ (YSZ). The laminate was cut into $1 \mathrm{~cm}$ diameter button cell configuration and sintered at $1450^{\circ} \mathrm{C}$ for $2 \mathrm{~h}$. The thickness of the electrolytes was approximately $110 \mu \mathrm{m}$. Gadolinium-doped ceria (GDC) was screen-printed on both sides of the YSZ substrate as a barrier layer between electrolyte (YSZ) and the electrodes to prevent undesired electrolyte-electrode reactions at elevated temperatures. The use of GDC impedes the undesired reaction 
between electrode and electrolyte, which is detrimental to performance of electrode. The effect of GDC barrier layer has been studied and reported by Yun et al. [79] that LSCF cathode with GDC barrier layer displayed lower polarization resistance of $0.45 \Omega \cdot \mathrm{cm}^{2}$ compared to $0.71 \Omega \cdot \mathrm{cm}^{2}$ of sample without GDC [79]. The GDC was sintered at $1400^{\circ} \mathrm{C}$ and held for $2 \mathrm{~h}$ to produce a final thickness of $\sim 5 \mu \mathrm{m}$. The cathode was later screenprinted on both sides of electrolyte membrane with a $\sim 15 \mu \mathrm{m}$ thick LSCF-GDC (50-50 vol \%) active layer and $\sim 40 \mu \mathrm{m}$ thick LSCF current collector. The cathode is then sintered at $1150^{\circ} \mathrm{C}$ and held for $2 \mathrm{~h}$.

Foamed Cathode: Transforming the direct foaming approach into a practical application for SOFC applications was a crucial step. It was a challenge to foam a thin layer of cathode over an electrolyte. The fabrication of the electrolyte, GDC barrier layer and anode (NiO/GDC) were completed as described above in the baseline section. For cathode foaming, an appropriate volume ratio of cathode material, polyurethane (PU) precursor mixture and ink vehicle (ethyl cellulose in terpineol - Johnson Matthey 63/2 medium) (solvent) was mixed to form a cathode ink. This cathode ink was prepared in an inert atmosphere in order to prevent the polymerization reaction to occur due the ambient moisture while mixing. The active layer of the cathode LSCF-GDC (50-50 vol\%) was screen-printed on both sides. The ink was then stenciled (at a thickness of 60-65 microns) as a thin film cathode over an electrolyte substrate in inert atmosphere with $0 \%$ relative humidity. The sample was then exposed to an aerosol mist of water sprayed over the immediate ambience on top of the cells to raise the humidity levels to $55-65 \%$ of relative humidity, which led to foaming of thin film of cathode over a substrate (YSZelectrolyte). The cell was then dried in oven at $75^{\circ} \mathrm{C} / 0.5 \mathrm{~h}$ and fired in a furnace using firing schedule described above. Fig. 4.1 shows an illustrative description of a foamed cathode symmetrical cell used for EIS in this work. 


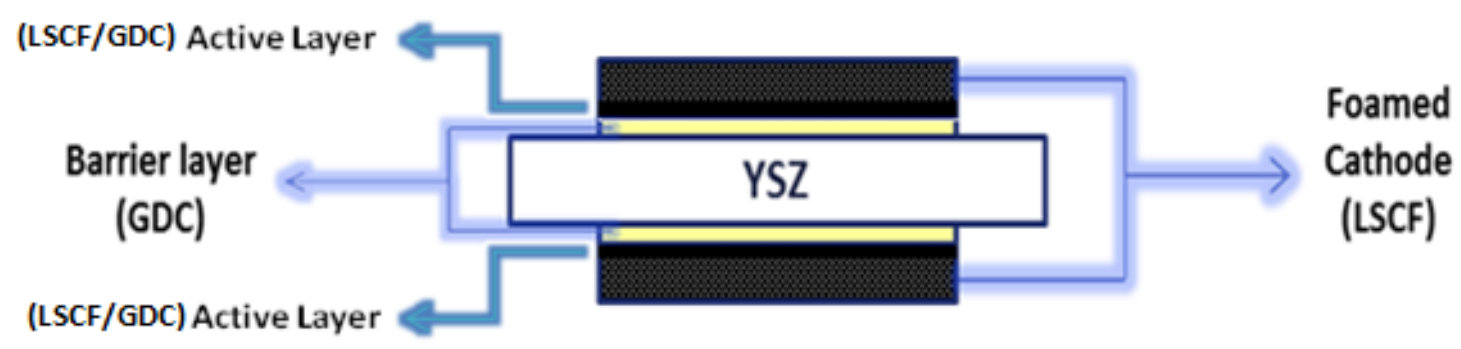

Figure 4.1: Schematic drawing of an electrolyte supported cathode symmetrical cell.

\subsubsection{Electrochemical Impedance Spectroscopy}

Electrochemical impedance spectroscopy (EIS) was performed on these electrolyte supported cathode symmetrical cells using a Solartron SI-1287 electrochemical interface and an SI-1260 impedance analyzer. The EIS is conducted at $750^{\circ} \mathrm{C}$ and $800^{\circ} \mathrm{C}$ under different loading conditions such as OCV, $100 \mathrm{~mA}$ and $300 \mathrm{~mA}$. The analysis and curve fitting of the data was then carried out using Z-View software. Bouwmeester et al. [80] studied the transport coefficients such as surface exchange and diffusion coefficients of oxygen for $\mathrm{La}_{0.6} \mathrm{Sr}_{0.4} \mathrm{Co}_{1-\mathrm{y}} \mathrm{Fe}_{\mathrm{y}} \mathrm{O}_{3-\delta}$ ( $\mathrm{y}=0.2,0.5$ and 0.8 ) using conductivity relaxation technique measured between $600-800^{\circ} \mathrm{C}$ in oxygen partial pressure between $10^{-4}$ to 1 bar. This work displayed decrease in both surface exchange and diffusion coefficients of oxygen with decrease in oxygen partial pressure below $10^{-2}$, from which it is derived that better kinetics can be observed in LSCF at atmospheric pressure. In this work, EIS is performed under supply of ambient air at standard atmospheric pressure of oxygen.

\subsubsection{Fuel Cell Testing}

The fuel cell testing was performed within a button cell fuel cell test stand. The cell is mounted on an alumina fixture with a pair of $9 \times 9 \mathrm{~mm}$ platinum strips (100 mesh woven) as current collectors and leads were drawn out using 0.016 " diameter platinum 
wire and attached on both cathode and anode using inks of LSCF and NiO metal as contact pastes, respectively. The cell was then heated to $600^{\circ} \mathrm{C}$ at $1{ }^{\circ} \mathrm{C} / \mathrm{min}$. under $60 \mathrm{sccm}$ of argon gas within the anode chamber for the reduction of anode and ambient air in the cathode chamber. Later, the cell is heated to $800^{\circ} \mathrm{C}$ at $2^{\circ} \mathrm{C} / \mathrm{min}$. under $30 \mathrm{sccm}$ of each argon and moist hydrogen on anode while cathode remains under ambient air. After the cell reaches $800^{\circ} \mathrm{C}$, the cell was loaded under $100 \mathrm{sccm}$ of moist $\mathrm{H}_{2}$ on the NiO/GDC anode. The current-voltage (I-V) and power density curves were obtained during the fuel cell testing. Fig 4.2 illustrates the typical button cell configuration used for testing through this work.

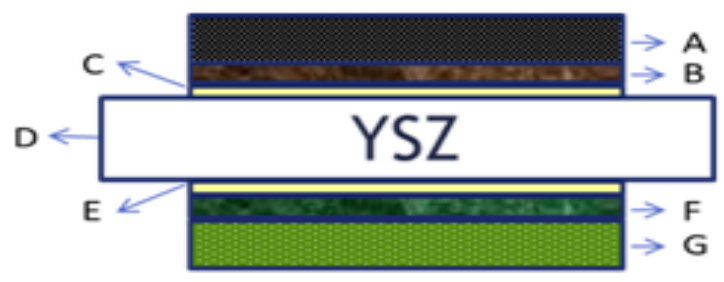

Electrolvte Supported Cells:

$\mathrm{A} \rightarrow \mathrm{LSCF}$-foamed (Current Collector layer) $-65-80 \mu \mathrm{m}$

$\mathrm{B} \rightarrow \mathrm{LSCF} / \mathrm{GDC}$ (Active layer) $-15 \mu \mathrm{m}$

$\mathrm{C} \rightarrow \mathrm{GDC}$ (Barrier layer) $-5 \mu \mathrm{m}$

$\mathrm{D} \rightarrow \mathrm{YSZ}-$ Electrolyte $-120-150 \mu \mathrm{m}$

$\mathrm{E} \rightarrow \mathrm{GDC}$ (Barrier layer) $-5 \mu \mathrm{m}$

$\mathrm{F} \rightarrow \mathrm{NiO} / \mathrm{GDC}$ (Active layer) - $15 \mu \mathrm{m}$

$\mathrm{G} \rightarrow \mathrm{NiO} / \mathrm{GDC}$ (Current Collector layer) $-40 \mu \mathrm{m}$

Figure 4.2: Configuration of electrolyte supported full SOFC. 


\subsection{Results}

\subsubsection{Effect of polymer precursors composition on foamed cathode over the YSZ electrolyte}

The results from the preceding chapter described work that was completed by foaming within a cup and not on a substrate (i.e. bulk). In this work, utilizing the same process was described, but completing this reaction on a substrate, where the free-foam structure must bond to the substrate after thermal processing (and retain the microstructure previously described).

The following section discusses whether the precursor compositions and processing parameters used for bulk foaming process produces similar microstructures when deposited/foamed as a thin film over a substrate. Hence, the same foaming conditions were evaluated to investigate the processing-microstructure relationship when deposited on the electrolyte-supported cathode structure. The effect of polymer precursor composition on the foamed cathode with $70 \%$ solids loading over the YSZ substrate, sintered at $1150^{\circ} \mathrm{C}$ is shown in Figure 4.3. The results from the cathode foamed over substrate followed a similar trend to previous results seen for cathode foamed in bulk, but at different (smaller) scale due to foaming is done on thin films of 60-70 microns thickness. The pores have higher porosity (around 46\%) for all foamed samples than baseline (32\%) and pores with foamed cathode have higher circularity factor compared to baseline. The mean pore area $\left(11.5 \mu \mathrm{m}^{2}\right)$ of the cathode foamed with $8: 4: 1$ (standard composition) around $22 \%$ higher than those of other compositions 9:4:1, 8:4:2 and baseline $\left(9.5,9.7\right.$ and $\left.8 \mu \mathrm{m}^{2}\right)$.

The mean pore area of foamed compositions was mapped against the region across the cathode thickness in Fig 4 (a). This shows a gradient in mean pore area across the thickness of the cathode. It appears that the pores at the top of the cathode (the region farther from substrate) have higher mean pore area than those nearer to the substrate under similar porosity levels. The reason for which can be explained as the possibility of the coalesced bubbles travel upwards due to buoyancy effect and higher bubble pressure 
leaving fine and discrete pores behind at bottom. Fig 4(b) clearly displays that circularity factor and porosity remained under similar levels at both near and farther from electrolyte. This is a desirable feature in SOFC applications as the similar porosity level here implies that there are bigger pores in the cathode at a location away from the electrolyte; this supports better gas diffusion where it is vital and higher number of smaller pores closer to the electrolyte enhancing the triple phase boundary as well ensuring the better adhesion to the substrate. Large pores close to the electrolyte also may not be optimal for structural considerations, since this large collection of void space may lead to low bonding strength at the interface. In the end, this may than lead to a delamination of the cathode layer due to weak adhesion. Back-scattered SEM micrograph displaying a LSCF cathode film deposited and in situ foamed on YSZ substrate at a 70\% solids loading and 8:4:1 polymer precursor's composition (sintered at $1150^{\circ} \mathrm{C}$ ) is shown in Fig 4.5. 


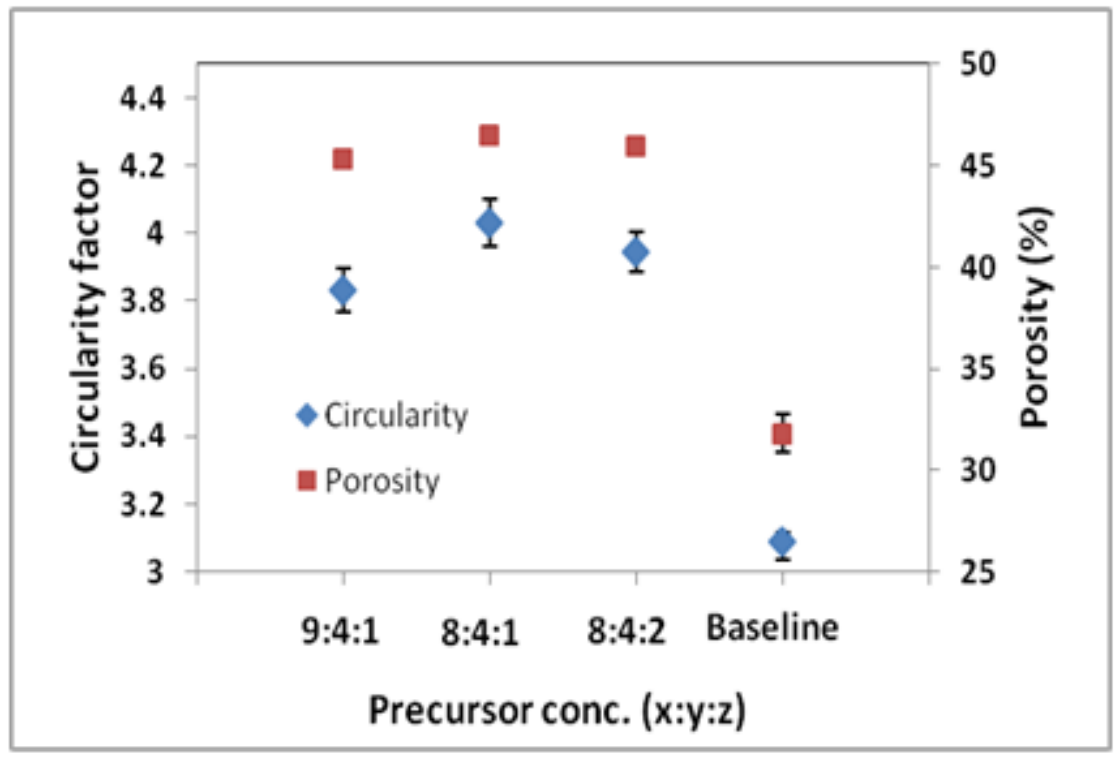

(a)

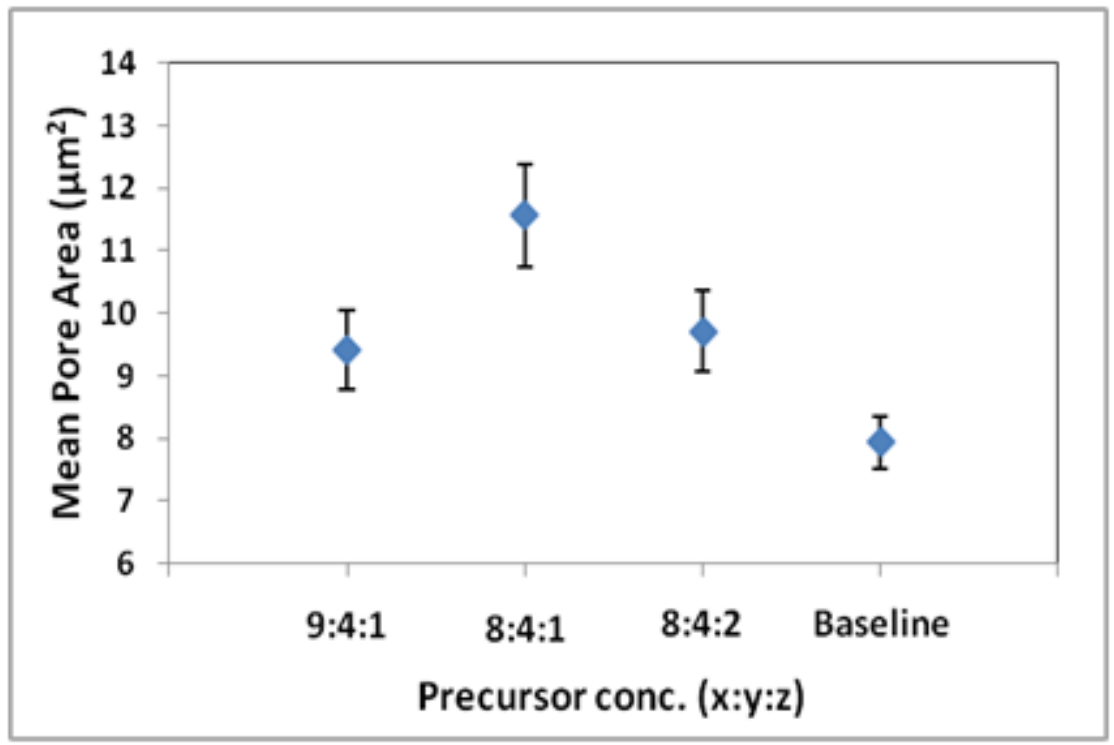

(b)

Figure 4.3: Effect of polymer precursor's composition on foamed cathode with $70 \%$ solids loading over the YSZ substrate, sintered at $1150^{\circ} \mathrm{C}$. 


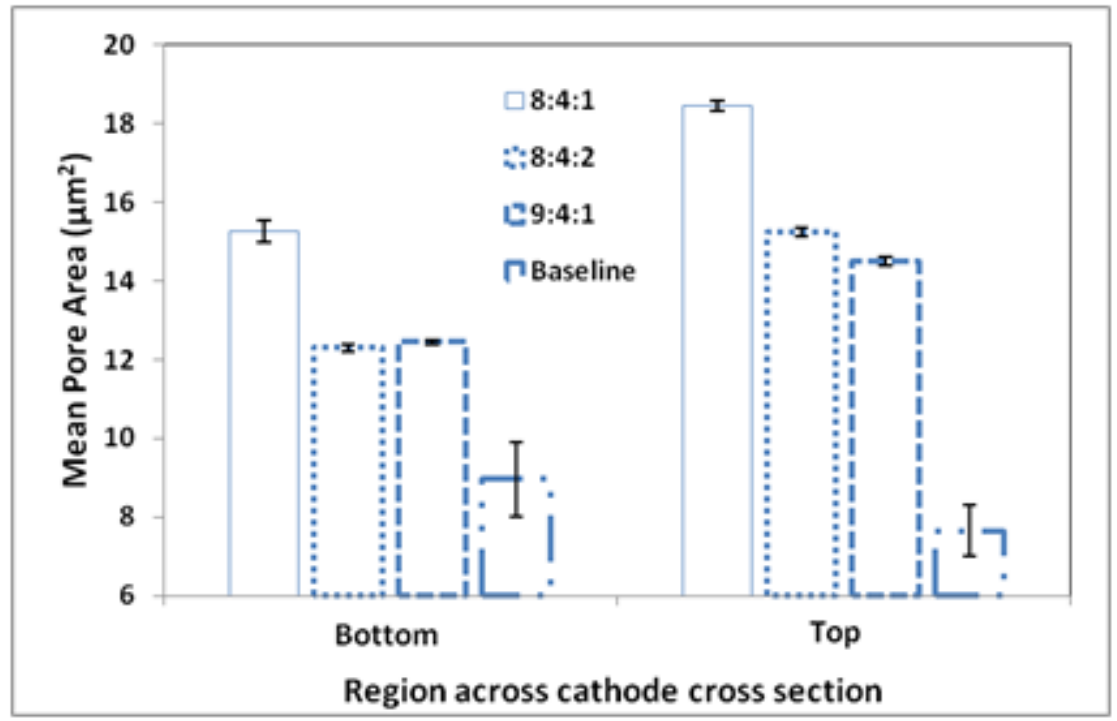

(a)

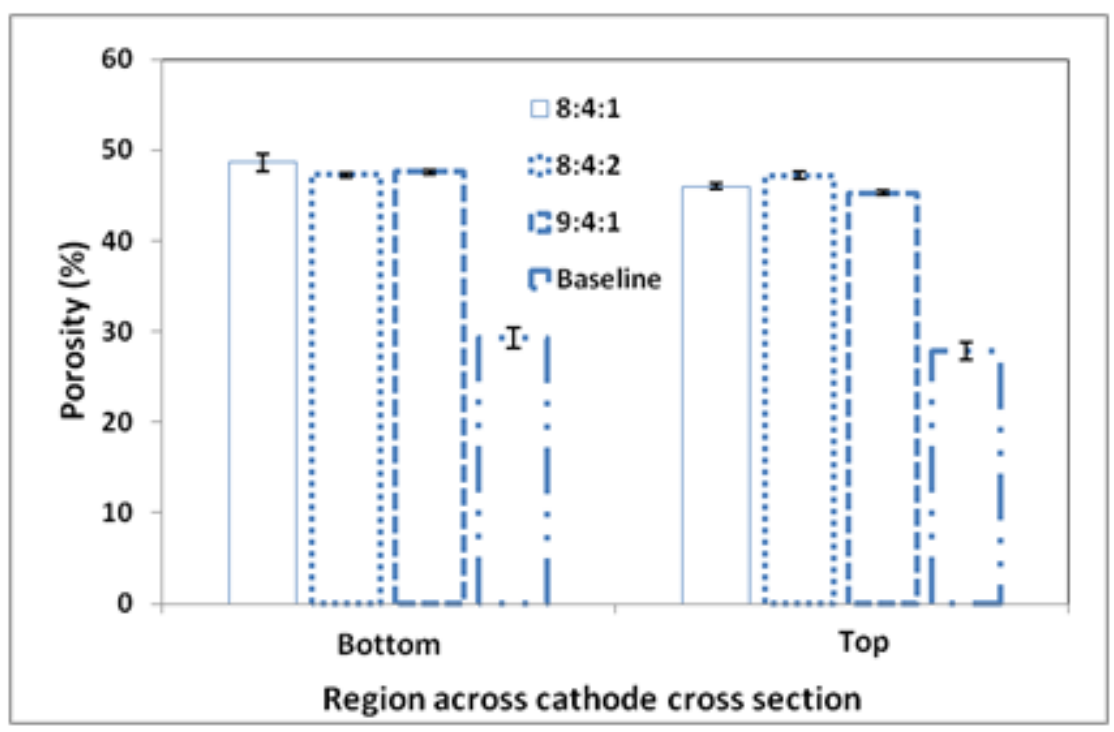

(b) 


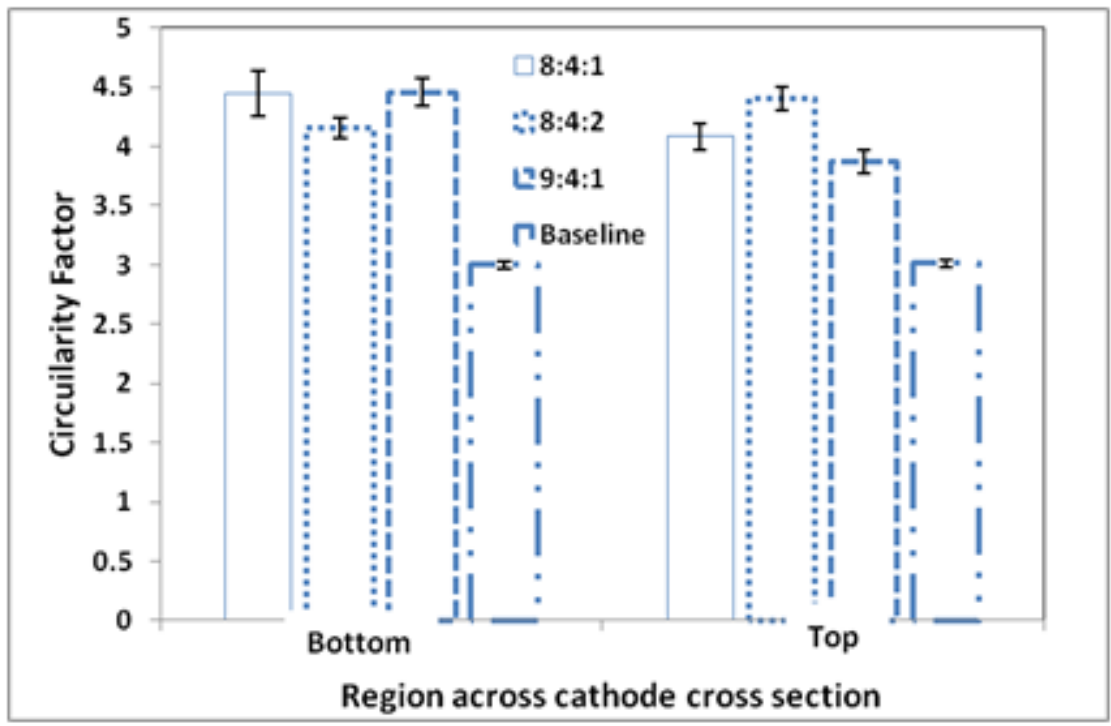

(c)

Figure 4.4: Across the thickness of the cathode (nearer and farther from electrolyte), effect of polymer precursor's composition on foamed cathode with $70 \%$ solids loading over the YSZ substrate, sintered at $1150^{\circ} \mathrm{C}$. 


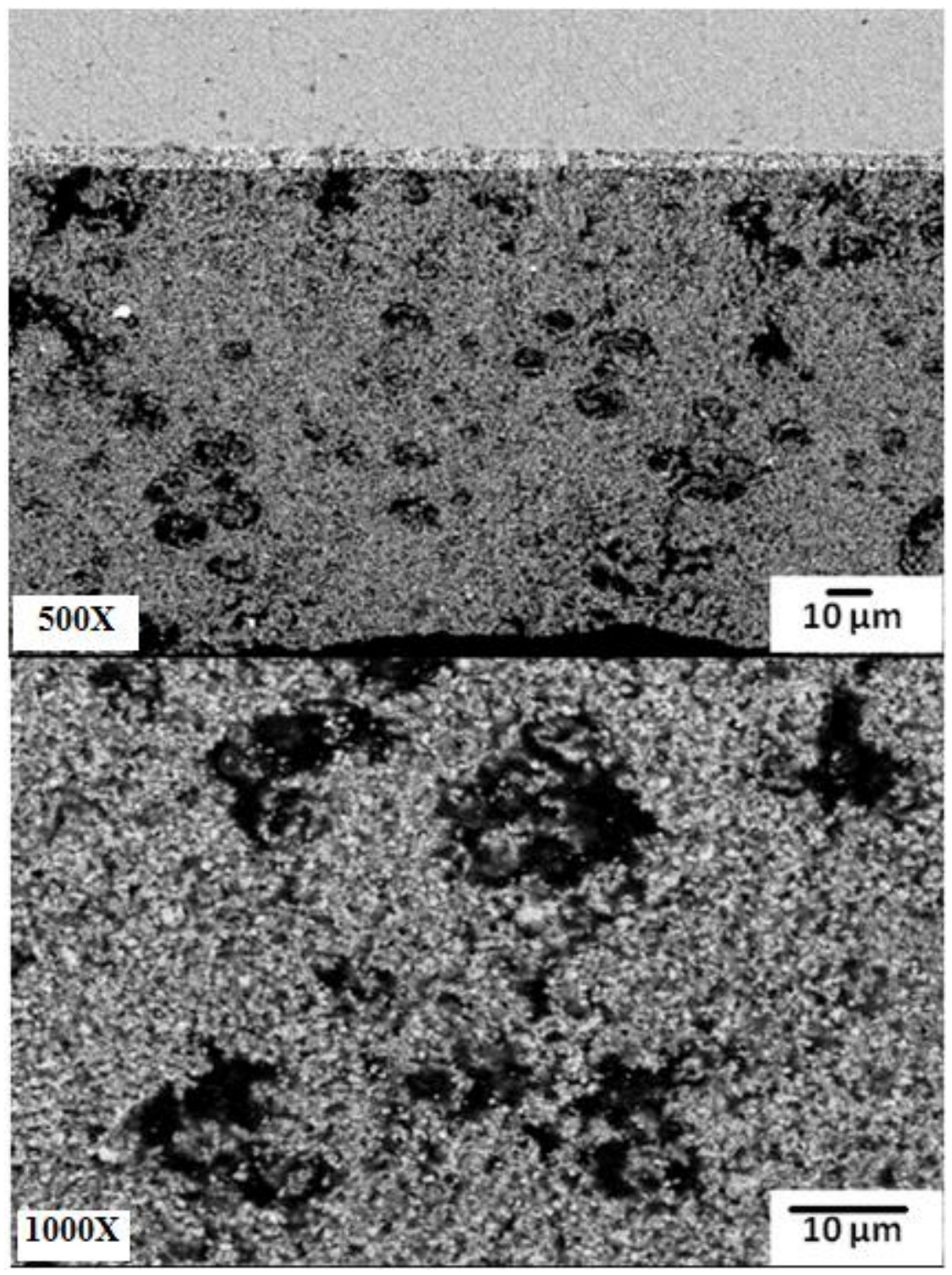

Figure 4.5: Back-scattered SEM micrograph of LSCF cathode film deposited and in-situ foamed on YSZ substrate at a 70\% solids loading and 8:4:1 polymer precursor's composition (sintered at $1150^{\circ} \mathrm{C}$ ) shown at lower and higher magnifiacations. 


\subsubsection{Electrochemical Testing}

Electrolyte-supported cathode symmetrical cells with three different precursor compositions 9:4:1, 8:4:1 and 8:4:2 (precursor: PEG 200: surfactant) volume ratios along with baseline (un-foamed) cathode, were tested through electrochemical impedance spectroscopy (EIS).

Based on image analysis of the cross-sectional SEM micrographs, it was learned that the sample with 8:4:1 (precursor: PEG 200: surfactant) ratio displayed a higher mean pore area with higher concentration of interconnected porosity. This type of microstructure is desirable for potentially enhanced oxygen reduction kinetics; therefore, this sample should display a lower level of electrode polarization. This cathode microstructure was expected to have a larger concentration of TPBs with low resistance to gas diffusion. Fig. 4.6, with the foamed sample with 8:4:1 (precursor: PEG 200: surfactant) precursor composition, displayed $\sim 40 \%$ and $\sim 50 \%$ less polarization than baseline cell at $750^{\circ} \mathrm{C}$ and $800^{\circ} \mathrm{C}$, respectively. The results were normalized by subtracting the ohmic resistance to account for different electrolyte thicknesses which is considered to be a major contributor to the ohmic resistance. The total polarization for the entire symmetrical cell displayed a combined electrode polarization of $0.16 \Omega-\mathrm{cm}^{2}$ (thus, a singular cathode polarization of $0.08 \Omega-\mathrm{cm}^{2}$ ) at $800^{\circ} \mathrm{C}$. The polarization resistance of the baseline $\left(0.36 \Omega-\mathrm{cm}^{2}\right)$ was in agreement with $0.45 \Omega-\mathrm{cm}^{2}$ [81] and $0.4 \Omega-\mathrm{cm}^{2}$ [82], various symmetrical cell tests with LSCF cathode reported in literature at $800^{\circ} \mathrm{C}$ under OCV. Polarization of the foamed sample with 8:4:1 precursor composition was lower at all loadings and temperatures when compared to other compositions in this work. The other polymer precursor compositions for foamed cathode 9:4:1 and 8:4:2 (precursor: PEG 200: surfactant) displayed similar polarization to the baseline at $800^{\circ} \mathrm{C}$ as $0.4 \Omega$ -

$\mathrm{cm}^{2}, 0.38 \Omega-\mathrm{cm}^{2}$ and $0.3 \Omega-\mathrm{cm}^{2}$ at open circuit voltage, $100 \mathrm{~mA}$ and $300 \mathrm{~mA}$ respectively. 

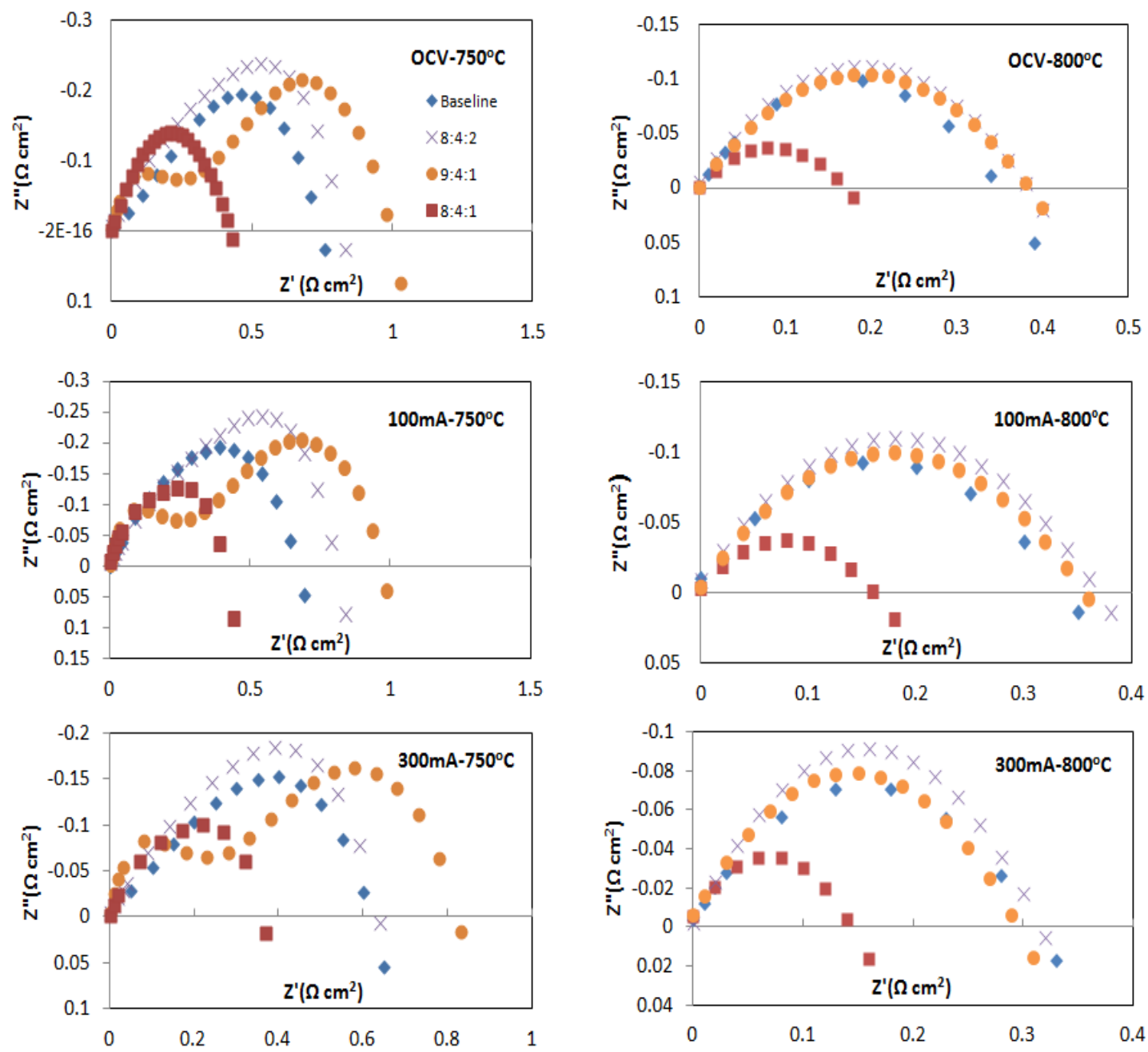

Figure 4.6: EIS spectrum of electrolyte-supported cathode symmetrical cells through EIS at $750^{\circ} \mathrm{C}$ and $800^{\circ} \mathrm{C}$ at $\mathrm{OCV}, 100 \mathrm{~mA}$ and $300 \mathrm{~mA}$ loadings.

\subsubsection{Fuel Cell Testing}

The structure of the SOFC cell fabricated with a foamed cathode is shown in Fig. 4.2. The cathode was processed using the 8:4:1 precursor composition ratio. The foamed cathode SOFC and identical cell fabricated with standard cathode (baseline cell) were tested under moist hydrogen on anode and ambient air flow on cathode at $800^{\circ} \mathrm{C}$. The performance of the SOFC with in situ foamed cathode showed a significant improvement over the baseline cell. The cell with foamed cathode displayed a maximum power density 
of $514 \mathrm{~mW} / \mathrm{cm}^{2}$, which is $43 \%$ higher than baseline cell $\left(360 \mathrm{~mW} / \mathrm{cm}^{2}\right)$ as shown in Fig.4.7.

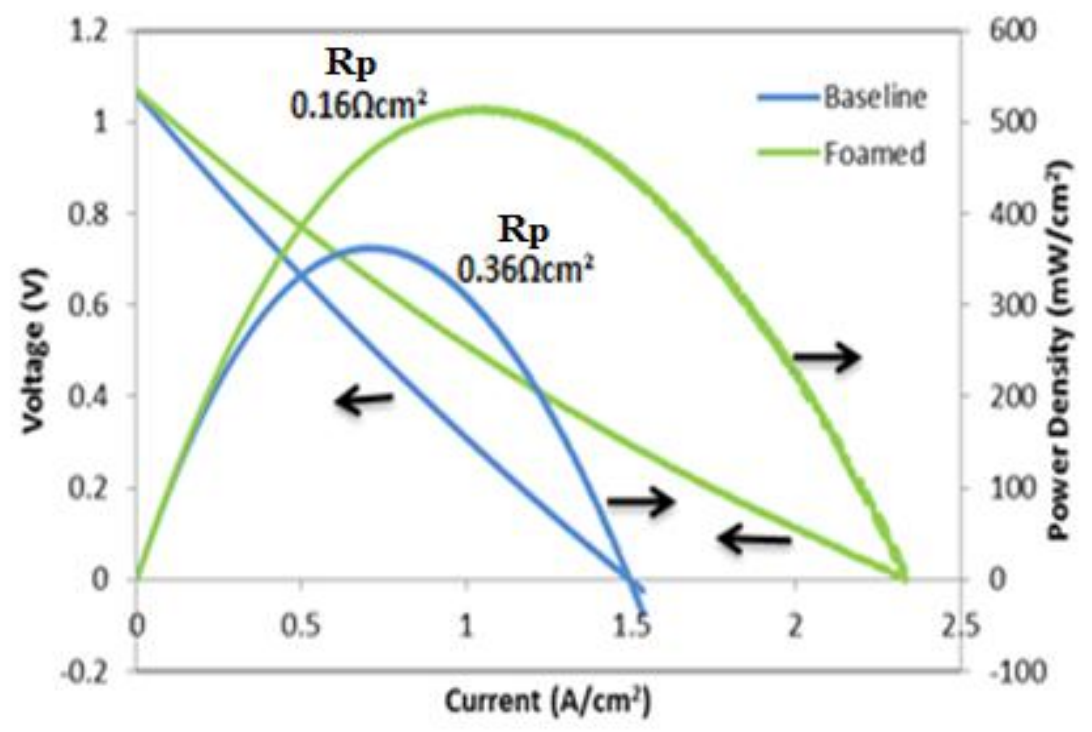

Figure 4.7: I-V and Power density curves of foamed and baseline cells at $800^{\circ} \mathrm{C}$ with moist $\mathrm{H}_{2}$ as fuel.

\subsection{Conclusion:}

The application of the direct foaming method on the cathode thick films deposited on YSZ substrates was investigated to ensure its practical application in SOFC processing. The change in the polymer precursor compositions were examined on the substrate, and the resultant microstructures were shown to produce similar microstructures as those previous produced in the bulk foaming (previous chapter). The electrochemical performance of in situ foamed cathode was investigated through EIS and fuel cell testing which displayed $50 \%$ decrease in the polarization resistance and $43 \%$ increase in the peak power density when compared to the baseline composition. 


\section{Chapter 5: EFFECT OF FOAMED CATHODE STRUCTURE ON NANO-CATALYST INFILTRATION}

\subsection{Back ground and Objective}

The need for high operating temperatures $\left(600^{\circ} \mathrm{C}-800^{\circ} \mathrm{C}\right)$ for reaction mechanisms is one of the most limiting parameter in SOFC commercialization. The technology for addition of catalytic functionality to enhance the reaction kinetics in electrodes of SOFCs has been highly investigated. The electro-catalytic nano-particles impregnation into sintered cathode is considered to be an advantageous approach in enhancing the electrode performance [83]. Uchida et al. [84] reported the performance enhancement in LSM cathodes through Pt nano-catalyst impregnation with achieving higher reaction rates of the oxygen reduction mechanism. The addition of nano-particles of ion conducting SDC into commercial porous cathodes through impregnation process was shown by $\mathrm{Li}$ et al. [85] to yield the reduction in polarization by a factor of 6 to 13 at $700-1000^{\circ} \mathrm{C}$, respectively. The addition of noble metals such as $\mathrm{Pt}, \mathrm{Ag}$, amd $\mathrm{Pd}$ as catalysts to a baseline LSM cathode for the enhancement of the electrochemical performance were demonstrated by Haanapel et al. [86] on anode supported cells. An increase of 8\% in maximum power density by Pt impregnated 2\% A-site deficient LSCF cathode over unimpregnated sample was reported by Huang et al. [87] at $800^{\circ} \mathrm{C}$. The above review literature demonstrates that the effective impregnation of the electro-catalyst in SOFC cathodes can result in improve electrochemical performance.

The objective of this work is to evaluate the processing efficiency and electrochemical performance of direct foamed cathode thick films which were impregnated with platinum (Pt) nano-catalyst. It is known from the previous study of this work that the foamed cathode possesses a porous microstructure with wide pore size distribution and interconnected porosity. Because of such microstructure, it retains an inherent strength of holding nano-catalyst well dispersed and uniformly covered over the 
grain structure within the cathode, potentially over that of the baseline cathode microstructure. The goal is to produce a uniformly coated cathode interior where the nano-catalyst is homogenously distributed from the peripheral region to inner region nearer to the substrate. As porosity is higher, the nano-catalyst infiltrated will form a uniform layer over the grain surface while retaining its porosity without appreciable drop of filling capabilities of all small pores throughout the microstructure. All of the above mentioned features are desirable for the enhanced performance of the electrode.

\subsection{Experimental}

\subsubsection{Infiltration of Platinum nano catalyst}

The samples were made by depositing LSCF cathode film on an YSZ substrate with 70 vol\% solids loading and 8:4:1 polymer precursor composition and screen-printed baseline cathode, sintered at $1150^{\circ} \mathrm{C}$. A platinum precursor $\left(\mathrm{H}_{2} \mathrm{Pt} \mathrm{Cl}_{6} \cdot 6 \mathrm{H}_{2} \mathrm{O}\right)$ was soluble in water at 0.1 molar concentrations, and this solution was used for the impregnation process. This solution was impregnated into the cathode with micro-pipette $(10-100 \mu \mathrm{L}$, Eppendrof International). The calcination of the impregnated cathode was performed $850^{\circ} \mathrm{C}$ for $1 \mathrm{~h}$. The weight change of the impregnated structure was measured using a Voyager Pro sensitive balance at regular intervals until the catalyst reached $5 \mathrm{wt} \%$ of cathode initial weight. The SEM imaging and EDS analysis of the half cells with the above-mentioned compositions were performed using JEOL 7600 SEM microscope.

\subsubsection{Fuel Cell testing}

The fuel cell testing was performed within a button cell fuel cell test stand. The cell is mounted on an alumina fixture with a pair of $9 \times 9 \mathrm{~mm}$ platinum strips $(100 \mathrm{mesh}$ woven) and the current collectors and leads were drawn out using 0.016" platinum wire and attached on both the cathode and anode using inks of LSCF and Ni metal, respectively. The cell was then heated to $600^{\circ} \mathrm{C}$ at $1{ }^{\circ} \mathrm{C} / \mathrm{min}$. under $60 \mathrm{sccm}$ of argon gas within the anode chamber for the reduction of anode and ambient air in the cathode chamber. The cell was heated to $800^{\circ} \mathrm{C}$ at $2^{\circ} \mathrm{C} / \mathrm{min}$. with $60 \mathrm{sccm}$ of a $50 \% \mathrm{Ar}$ and $50 \%$ 
moist hydrogen fuel mixture on anode while cathode remains under ambient air. After the cell reaches $800^{\circ} \mathrm{C}$, the cell was loaded under $100 \mathrm{sccm}$ of moist $\mathrm{H}_{2}$ on the anode. The current-voltage (I-V) and power density curves were obtained during the fuel cell testing.

\subsection{Results}

\subsubsection{Weight analysis}

The careful tracking of the amount of precursor solution added in every impregnation step was measured. The final amount of precipitated catalyst was also measured after drying and calcination. This study compares the amount of catalyst that could be impregnated in a foamed and baseline cathode per infiltration step. This investigation was completed on the baseline sample and also on two foamed cathodes of similar composition as explained in experimental section above. The amount of the catalyst added into the microstructure per infiltration step after calcination is shown in Fig 5.1 (a). The primary calcinations were performed after the $1^{\text {st }}$ and $2^{\text {nd }}$ infiltration steps at $450^{\circ} \mathrm{C} / 1 \mathrm{~h}$, which resulted in the production of $\mathrm{Pt} / \mathrm{PtO}_{\mathrm{x}}$ and some carbonaceous content. The rest of the calcination steps after further infiltration steps were completed at $850^{\circ} \mathrm{C} / 1 \mathrm{~h}$. The catalyst weight was normalized as weight per volume to account for differences in thickness between the foamed and baseline cathodes. The foamed cathode showed the ability to hold a higher catalyst amount after every step. The figure shows that the microstructure displayed a $90 \%$ higher content at the final step. The dip after the $3^{\text {rd }}$ infiltration step is due to increase in calcination temperature which burns the remaining carbonaceous content. The amount of the precursor solution added per impregnation step is plotted in Fig. 5.1 (b) for the foamed and baseline samples. The results are well correlated with the above-discussed trend. These results explain that the interconnected and high porosity of foamed cathode helped in holding higher amounts of catalyst per infiltration step, which can reduce the infiltration steps and processing time. 


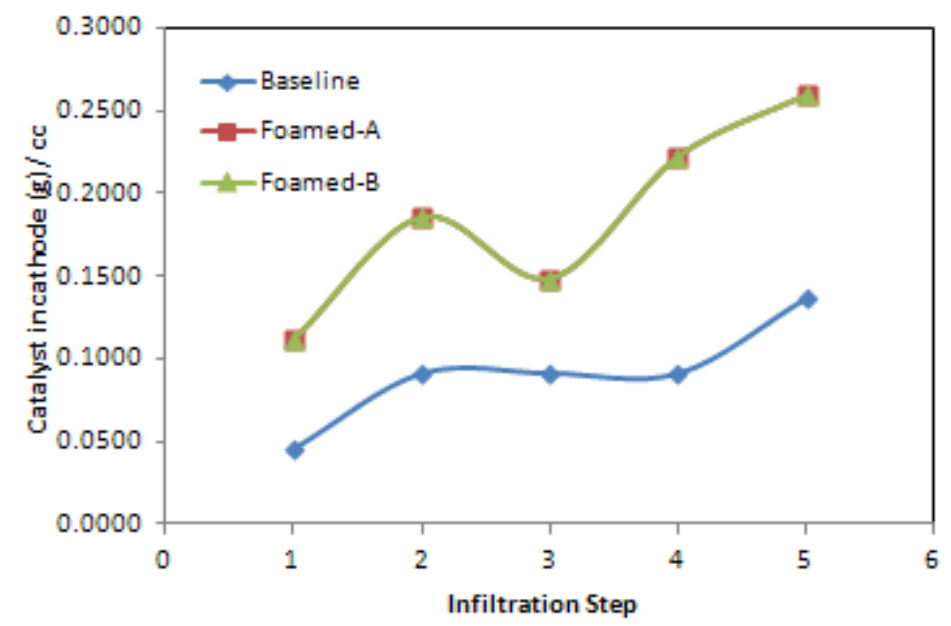

(a)

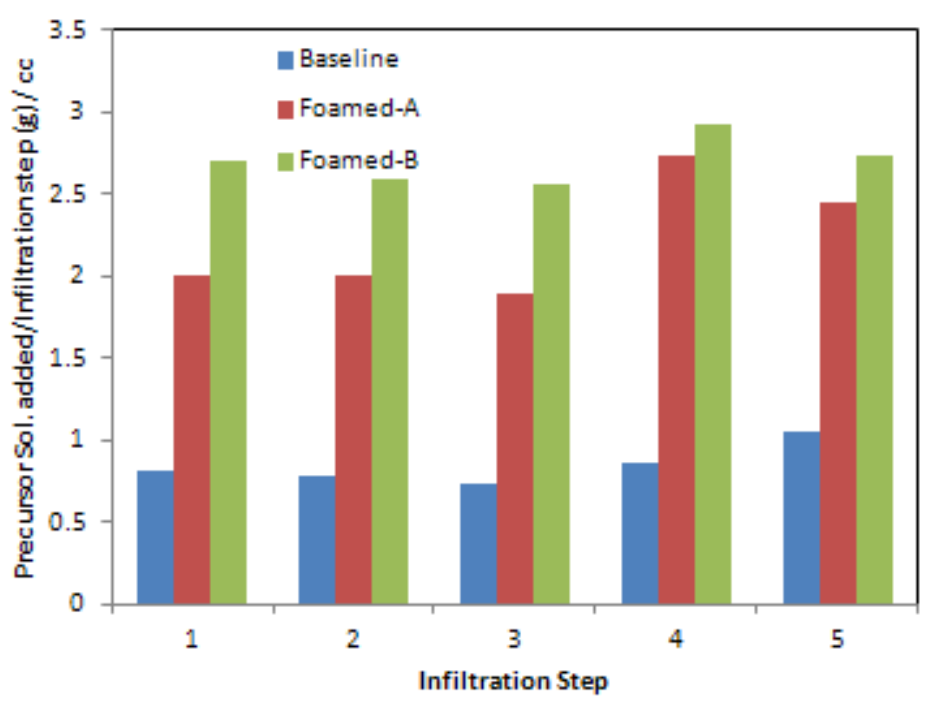

(b)

Figure 5.1: a) Amount of catalyst in cathode per infiltration step (calcined at $\left.\left.850^{\circ} \mathrm{C} / 1 \mathrm{~h}\right), \mathrm{b}\right)$ The amount of precursor solution could be added per infiltration step (no calcination) 


\subsubsection{SEM and EDS analysis}

The cross-sectional SEM images of the fractured surface of a nano-catalyst (5 wt\%) impregnated LSCF cathode, which was foamed over an YSZ electrolyte is shown in Fig. 5.2. The images were taken across the thickness of the cathode from the LSCF cathode top surface (away from the electrolyte), middle and bottom (electrode-electrolyte interface) regions. In addition to the SEM images, EDS spectra were taken from various locations in the same regions (Table 5.1, 5.2, and 5.3) to confirm the Pt presence. This analysis displayed that a very low percentage of Pt was dispersed through the thickness of the baseline cathode, and instead concentrated at the surface of the film. In contrast, the SEM and EDS data showed a strong presence of the Pt throughout the thickness of the foamed sample. Also, it was observed that agglomerations of the Pt formed in the baseline, while a fine dispersion of nano-particles were seen in the foamed sample. This agglomeration in the baseline sample may have been due to the lack of interconnected porosity and higher mean pore area. The baseline samples contained a homogenous mixture of finer porosity, which should have led to uniform wicking of the solution throughout the microstructure due to capillary action. Forced the solution's nanoparticles to concentrate at heterogeneous locations instead of homogeneous dispersion and agglomerate after precipitation which is not contributing to increase in active surface area. 


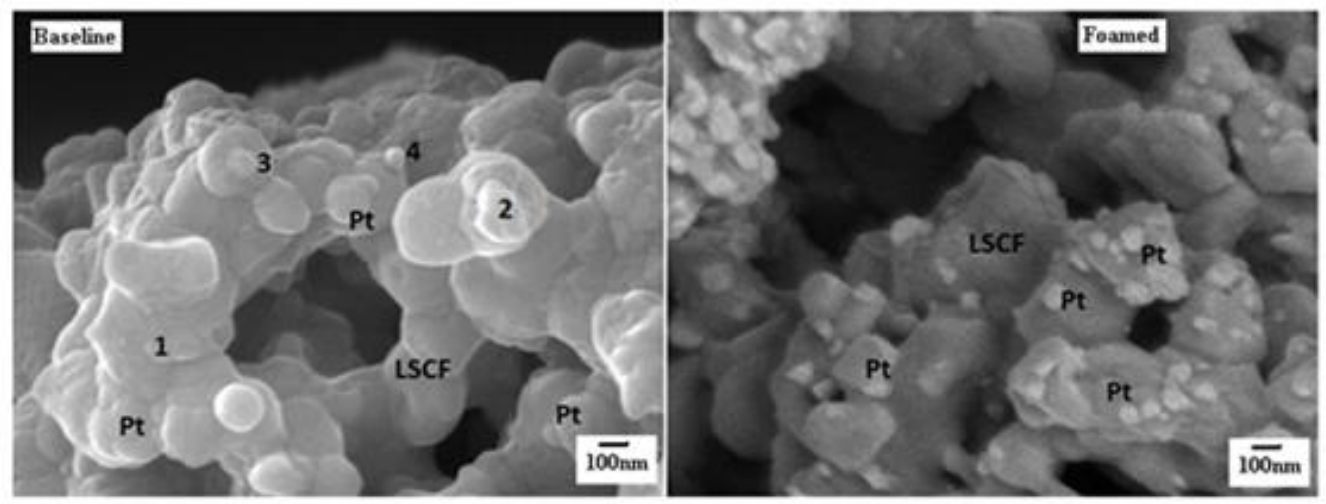

(a)

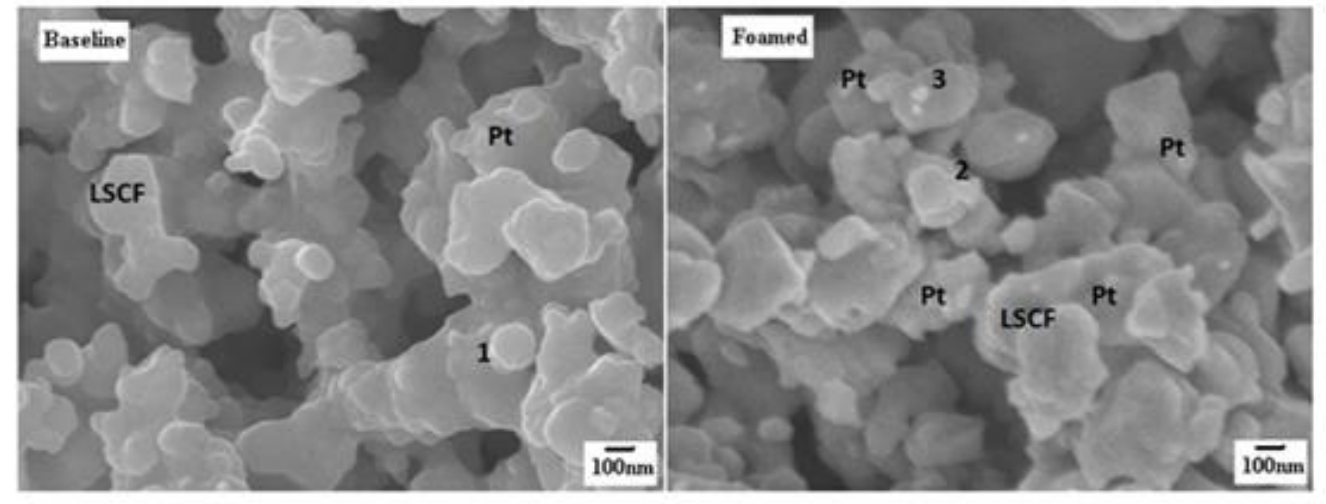

(b)

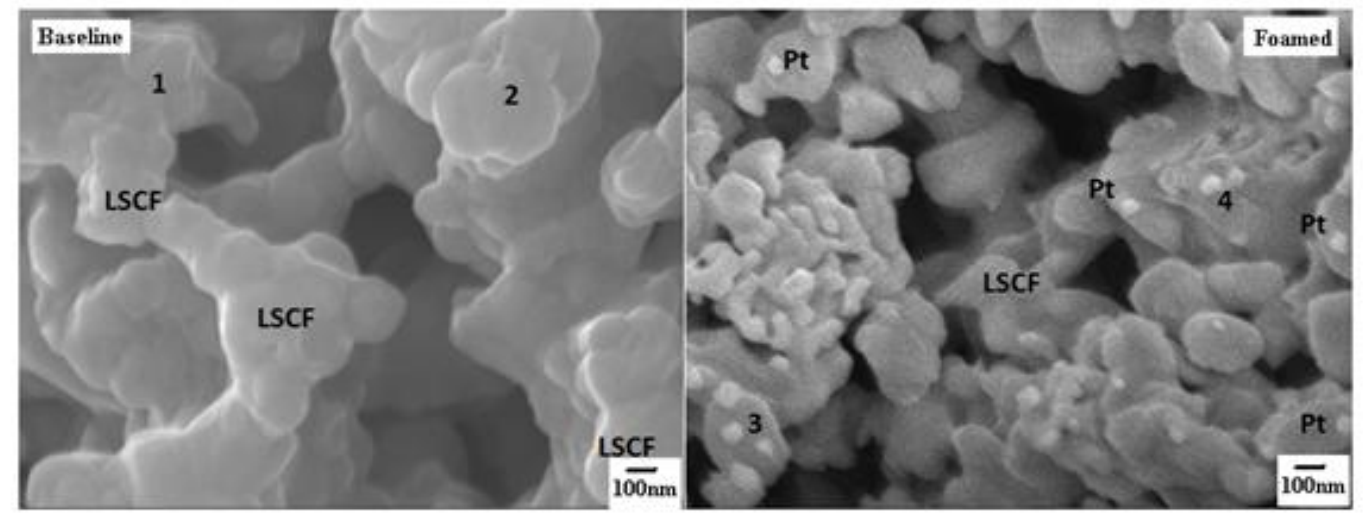

(c)

Figure 5.2: SEM images of fractures surface of the cross section of infiltrated foamed and baseline cathodes in a) top (away from electrolyte) b) middle and c) bottom (at electrode-electrolyte interface) 
Table 5.1: Atom\% of corresponding element (farther from eelctrolyte)

\begin{tabular}{ccccccc}
\hline & $\mathrm{O}$ & $\mathrm{Fe}$ & $\mathrm{Co}$ & $\mathrm{Sr}$ & $\mathrm{La}$ & $\mathrm{Pt}$ \\
\hline 1 & $\mathbf{6 5 . 7 6}$ & $\mathbf{1 0 . 9 5}$ & $\mathbf{5 . 1 6}$ & $\mathbf{6 . 8 0}$ & $\mathbf{9 . 3 1}$ & $\mathbf{2 . 0 2}$ \\
2 & $\mathbf{5 4 . 9 4}$ & $\mathbf{1 5 . 4 6}$ & $\mathbf{6 . 0 7}$ & $\mathbf{1 0 . 0 1}$ & $\mathbf{9 . 7 0}$ & $\mathbf{3 . 8 3}$ \\
3 & $\mathbf{6 5 . 9 1}$ & $\mathbf{1 4 . 5 6}$ & $\mathbf{3 . 0 2}$ & $\mathbf{5 . 9 5}$ & $\mathbf{7 . 0 8}$ & $\mathbf{3 . 4 8}$ \\
4 & $\mathbf{7 0 . 2 9}$ & $\mathbf{9 . 2 6}$ & $\mathbf{4 . 5 3}$ & $\mathbf{5 . 1 8}$ & $\mathbf{6 . 4 9}$ & $\mathbf{4 . 2 6}$ \\
\hline
\end{tabular}

Table 5.2: Atom\% of corresponding element (midddle zone)

\begin{tabular}{ccccccc}
\hline & $\mathrm{O}$ & $\mathrm{Fe}$ & $\mathrm{Co}$ & $\mathrm{Sr}$ & $\mathrm{La}$ & $\mathrm{Pt}$ \\
\hline 1 & $\mathbf{5 6 . 5 2}$ & $\mathbf{1 7 . 0 4}$ & $\mathbf{5 . 2 5}$ & $\mathbf{7 . 1 6}$ & $\mathbf{1 2 . 6 1}$ & $\mathbf{1 . 4 1}$ \\
2 & $\mathbf{3 5 . 6 9}$ & $\mathbf{1 2 . 9 2}$ & $\mathbf{5 . 8 4}$ & $\mathbf{3 2 . 1 2}$ & $\mathbf{1 0 . 9 5}$ & $\mathbf{2 . 4 8}$ \\
3 & $\mathbf{4 6 . 2 6}$ & $\mathbf{2 . 4 4}$ & $\mathbf{3 . 4 4}$ & $\mathbf{2 7 . 2 6}$ & $\mathbf{1 1 . 2 4}$ & $\mathbf{9 . 3 6}$ \\
\hline
\end{tabular}

Table 5.3: Atom\% of corresponding element (cathodelelectrolyte interface zone)

\begin{tabular}{ccccccc}
\hline & $\mathrm{O}$ & $\mathrm{Fe}$ & $\mathrm{Co}$ & $\mathrm{Sr}$ & $\mathrm{La}$ & $\mathrm{Pt}$ \\
\hline 1 & $\mathbf{6 9 . 3 4}$ & $\mathbf{1 0 . 7 9}$ & $\mathbf{4 . 8 5}$ & $\mathbf{6 . 5 3}$ & $\mathbf{8 . 3 1}$ & $\mathbf{0 . 1 9}$ \\
2 & $\mathbf{6 7 . 9 5}$ & $\mathbf{1 2 . 3 5}$ & $\mathbf{4 . 1 7}$ & $\mathbf{6 . 0 3}$ & $\mathbf{8 . 8 0}$ & $\mathbf{0 . 7 0}$ \\
3 & $\mathbf{5 8 . 2 2}$ & $\mathbf{1 3 . 3 2}$ & $\mathbf{5 . 4 6}$ & $\mathbf{1 8 . 3 7}$ & $\mathbf{0 . 3 9}$ & $\mathbf{4 . 2 4}$ \\
4 & $\mathbf{5 4 . 3 3}$ & $\mathbf{2 1 . 7 3}$ & $\mathbf{4 . 4 7}$ & $\mathbf{1 1 . 2 7}$ & $\mathbf{6 . 0 8}$ & $\mathbf{2 . 1 2}$ \\
\hline
\end{tabular}

\subsubsection{Fuel cell testing}

The current-voltage-power performance of SOFC button cells which possessed a LSCF cathode impregnated with Pt nano-catalyst was measured. Identical SOFCs fabricated with the standard LSCF cathode (baseline cell) were tested under moist hydrogen on anode and ambient air flow on cathode at $800^{\circ} \mathrm{C}$. The performance of the SOFC with in situ foamed cathode showed a significant improvement over the baseline cell. The cell with the impregnated foamed cathode displayed a maximum power density of $593 \mathrm{~mW} / \mathrm{cm}^{2}$, which is $15.2 \%$ higher than un-infiltrated foamed cathode (514 
$\mathrm{mW} / \mathrm{cm}^{2}$ ). The SOFC baseline cell with a Pt-impregnated LSCF cathode displayed a maximum power density of $\sim 390 \mathrm{~mW} / \mathrm{cm}^{2}$ at $800^{\circ} \mathrm{C}$. This accounts for $\sim 8.33 \%$ higher performance value over that of an un-impregnated baseline cell $\left(\sim 360 \mathrm{~mW} / \mathrm{cm}^{2}\right)$, as shown in Fig 5.3. The improvement in the foamed cathode due to electro-catalyst impregnation is $7 \%$ higher when compared to the improvement in baseline, which can be attributed to the homogeneous dispersion of fine catalyst particles.

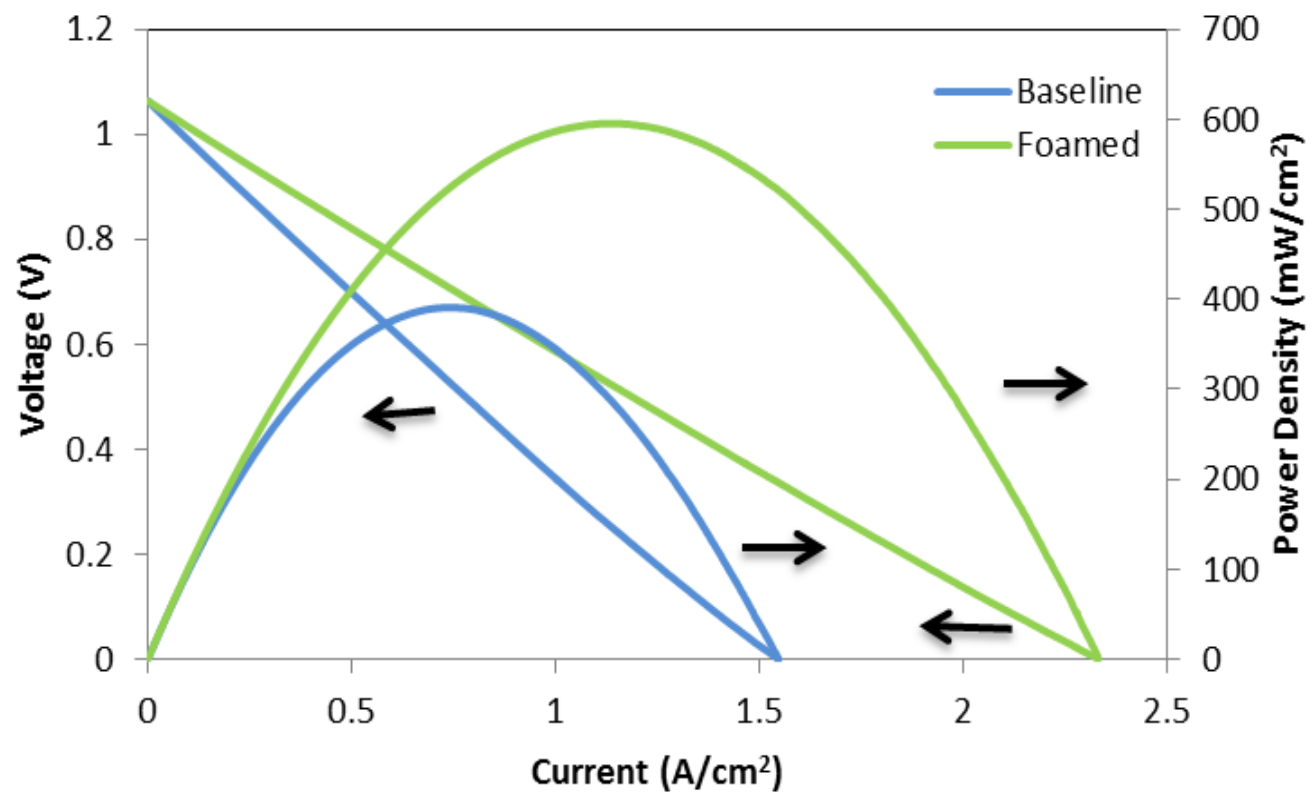

Figure 5.3: I-V and power density curves of SOFC button cells with impregnated foamed and baseline LSCF cathodes measured at $800^{\circ} \mathrm{C}$ with $\mathrm{H}_{2}$ fuel.

\subsection{Conclusion}

The dependency of the effectiveness of the electro-catalyst impregnation in SOFC cathode was investigated. The open pore structure with interconnected porosity has displayed the ability to allow homogeneous dispersion of the nano-particles which were impregnated across the cathode microstructure. This leads to fine dispersion of nanoparticles as a uni-layer over the pore surfaces increasing the effective active area for oxygen reduction which was confirmed by electrochemical testing. 


\section{Chapter 6: CONCLUSIONS AND FUTURE SCOPE}

\subsection{Conclusion}

An in situ foaming process was investigated for producing porous LSCF cathode structures with controlled porosity. The method was utilized to produce microstructures with a broad distribution of pore area with interconnected porosity and enhanced active surface area. The open pore structure with interconnected porosity increases the active surface area for the oxygen reduction mechanism in the cathode which enhances the electrochemical performance of the SOFC. The governing forces/factors for the bubble nucleation and growth such as surface tension, particle pressure against bubble and bubble expansion rate were carefully controlled through changes in compositions and materials of the processing parameters. The balance in the above-stated forces is needed to obtain the targeted microstructure of this work. Any imbalance in the surface tension or other factors that counter acts the bubble pressure leads to shrinkage or large coalescence of the nucleated bubbles (drainage due to buoyancy effect) that in-turn results the collapse of the foam before settling. Computational image analysis of backscattered SEM micrographs was used to determine the characteristics of the microstructure as a function of changes to key compositional and processing parameters. The corresponding processing parameters for the targeted microstructural properties of the work were derived through the quantitative analysis by Image J. The mean pore area is significantly higher and pores more elongated/interconnected (tortuous) for the sample with the stoichiometric composition - 8:4:1 (Precursor: PEG 200: Surfactant) at 70 vol\% solids loading, when sintered at $1150^{\circ} \mathrm{C}$; this result is in comparison to samples formed with alternate precursor compositions at similar solids loading.

The microstructure obtained from this foaming method yielded higher active surface area for oxygen reduction. In addition, the interconnected porosity assists in the diffusion of gas through the gradient porous structure across the thickness of the cathode, where the structure demonstrated more small pores at electrode-electrolyte interface and larger pores away from the electrolyte. This structure ensures better adhesion (limits 
delamination) to electrolyte while retaining the enhanced TPB. The foamed cathode has displayed a significant amount (43\%) of increase in peak power densities and decrease in

polarization resistance $(40 \%)$ over baseline cathode when operated at $800^{\circ} \mathrm{C}$. This structure also contributed in homogeneous dispersion of the nano-catalyst across the cathode covering the wide active surface area serving as the better backbone than baseline.

\subsection{Future Scope}

The work mainly focused on using an unconventional pore forming technique in the field of SOFCs which was not focused upon by previous researchers. This work conclusively defined the optimum processing parameters to in situ foam LSCF cathode bulk and thick film materials. The foamed cathode microstructure demonstrated an enhancement in the oxygen reduction mechanism, and thus, displayed a lower level of cathode polarization resistance. While the work resulted in processing and cathode performance advancements, many difficulties in applying the in situ foaming process for the fuel cell fabrication were uncovered. Some of these are as follows:

1) The reproducibility for producing the desired structure was found to vary due to the process intricacies. The foaming process needs customized conditions such as an inert atmosphere while the polymer precursor is mixed and the humidity of the environment must be carefully monitored during deposition. Therefore, a method for mixing and depositing the layers within a controlled moisture atmosphere must be researched.

2) The major limitation of the foamed cathode microstructure is its low mechanical strength, which limits the ability to incorporate the cathode film within compressed planar stacks.

3) A better understanding of methods of controlling the bubble size and distribution would be helpful in further controlling and engineering architectures that would further increase all interested properties. The effect of LSCF (cathode) particle 
size on the control of the reaction kinetics in the in situ foaming for bubble nucleation and growth should be investigated.

\begin{abstract}
Although the qualitative microstructural factors were correlated between the microstructure and the electrochemical properties, the true origin of the performance enhancement is still under question. The work only showed that by processing the materials in a certain way results in a specific microstructure, which then leads to a specific electrochemical performance. Some correlations were drawn from these relations, but the mechanism aligned with these specific microstructure-properties relations were not proven out.
\end{abstract}




\section{REFERENCES}

1. G. Tverberg, "World energy consumption since 1820 in charts - The Oil Drum" (2012).

2. "International Energy Outlook", DOE/EIA-0484(2011).

3. "US DOE, International Energy Annual: World Energy Overview" (1999).

4. "Power Surge: Energy use and emissions continue to raise, World Resources Institute", World Resources (1998-99).

5. “International Energy Agency”, May (2012).

6. H. A. Glenn, "Fuel Cell technology and Its Importance to Society", Tech Directions, Vol. 65 - Issue 1, 25-27 (2005).

7. A. J. Poche Jr., K. C. Ennis "Beyond Demonstration: The role of fuel cells in DoD's energy in strategy", US-DOE (2011).

8. “Accomplishments and Progress Sheet", US DOE (2011).

9. B. Norman; "Development of a Low Cost 3-10 kW Tubular SOFC Power System", Annual Merit Review Proceedings, US-DOE (2010).

10. "Pathways to Commercial Success: Technologies and Products Supported by the Fuel Cell Technologies Program", fuel cell Technologies (FCT) Program, USDOE (2011).

11. "Residential use of fuel cells on rise", Clean Technica, Retrieved November 4, 2011, from http://cleantechnica.com/2011/11/04/residential-use-of-fuelcells-on-the-rise/.

12. T. Malkow, "SOFC in Brief", Fuel Cells and Hydrogen Energy, I, 3-12, DOI: 10.1007/978-1-4020-6995-6_1 (2008).

13. R. Bove, S. Ubertini (eds.), "Modeling Solid Oxide Fuel Cells - Methods, Procedures and Techniques", 3-12 (2008).

14. “Current Technology, Energy efficiency and renewable energy”, Fuel Cells, US-DOE, retrieved August 03, 2011, from http://www1.eere.energy.gov/hydrogenandfuelcells/fuelcells/fc_types.html

15. S. B. Adler, "Factors Governing Oxygen Reduction in Solid Oxide Fuel Cell Cathodes", Chem. Rev., 104, 4791-4843 (2004). 
16. R. O. Hayre, D. M. Barnett, F. B. Prinz, "The Triple Phase Boundary - A Mathematical Model and Experimental Investigation for Fuel Cells", Journal of The Electrochemical Society, 152 (2) A439-A444 (2005).

17. A. V. Virkar, "SOFC Materials and Processing Issues", SECA Core Technology Program Workshop, February 14 (2001).

18. J.O'M. Bockris, S. Srinivasan, "Fuel Cells: Their Electrochemistry", McGraw-Hill, New York (1969).

19. P. Kuchonthara, S. Bhattacharya, A. Tsutsumi, "Energy recuperation in solid oxide fuel cell (SOFC) and gas turbine (GT) combined system", Journal of Power Sources, 117, Issues 1-2, Pages 7-13 (2003)

20. J. Larminie, A. Dicks, "Fuel Cell Systems Explained", Wiley, London (2000).

21. S.C. Singhal, K. Kendall, "High Temperature SOFCs - Fundamentals, Design and Applications", Elsevier Ltd. (2004).

22. E.L. Cussler, "Diffusion: Mass Transfer in Fluid Systems", Cambridge University Press, Cambridge (1984).

23. A. B. Stambouli , E. Traversa , "Solid oxide fuel cells (SOFCs): a review of and environmentally clean and efficient source of energy"; Renewable and Sustainable Energy Reviews, 6, 433-455 (2002).

24. C. Sun, R Hui, J. Roller, "Cathode materials for solid oxide fuel cells: a review", J Solid State Electrochem., [14], 1125-1144 (2010).

25. J. W. Fergus, R. Hui, X. Li, D.P. Wilkinson, J. Zhang, "Solid Oxide Fuel Cells - Materials Properties and Performance”, CRC Press (2009).

26. S. C. Singhal, "Solid Oxide Fuel Cells - Electrochemistry Encyclopedia", (2008).

27. J. Fleig, J. Maier, "The polarization of mixed conducting SOFC cathodes: Effects of surface reaction coefficient, ionic conductivity and geometry; Journal of the European Ceramic Society", 24[6], 1343-1347 (2004).

28. R. J. Cava, "Perovskite Structure and Derivatives", Cava Lab - Solid State Chemistry Research Group. 
29. A. S. Bhalla, R. Guo and R. Roy, "The perovskite structure - a review of its role in ceramic science and technology”, Mat. Res. Innovat., 4, 3-26 (2000).

30. K. Nomura, S. Tanase, Solid State Ionics 98:229, (1997).

31. R. J. A. M. Van, E. H. P. Cordfunke, J Solid State Chem., 93:212 (1991).

32. F. Zheng, L. R. Pederson, "Phase Behavior of Lanthanum Strontium Manganites", Journal of The Electrochemical Society, 146 [8], 2810-2816 (1999).

33. J. Mizusaki et al.., Solid State Ionics, 132:167-180, (2000).

34. C. C. T. Yang, W. C. J. Wei, A. Roose, "Electrical conductivity and microstructures of $\mathrm{La} 0.65 \mathrm{Sr} 0.3 \mathrm{MnO} 3-8 \mathrm{~mol} \%$ yttria-stabilized zirconia", Materials Chemistry and Physics (2003).

35. S. Carter, A. Seluk, R.J. Chater, J. Kaida, J.A. Kilner, B.C. Steele, Solid State Ionics, 53-56:597-605 (1992).

36. L. W. Tai, M. M. Nasrallah, H. U. Anderson, D. M. Sparlin, S. R. Sehlin, Solid State Ionics, 76, 259-271 (1995).

37. L. W. Tai, M. M. Nasrallah, H. U. Anderson, D. M. Sparlin, S. R. Sehlin, Solid State Ionics, 76, 273-283 (1995).

38. A. Mai, M. Becker, W. Assenmacher, F. Tietz, E. Ivers - Tiffee, D. Stover, W. Mader, Solid State ionics 177, 1965 (2006).

39. F. Tietz, A. Mai, D. Stover, "From powder properties to fuel cell performance - A holistic approach for SOFC cathode development", Solid State Ionics $179,1509-1515$ (2008).

40. A. Mai, V. A. C. Hannappel, S. Uhlenbruck, F. Tietz, D.Stover, Solid State Ionics, 176, 1341(2005).

41. A. Petric, P. Huang, F. Tietz; Solid State Ionics, 135, 719-725 (2000).

42. Q. Xu, D. P. Huang, W.Chen, F. Zhang, B. T. Wang, Journal of Alloys and Compounds 429, 34-39 (2007).

43. H. J. M. Bouwmeester, M. W. D. Otter, B. A. Boukamp, “Oxygen transport in LSCF”, Journal of Solid State Electrochemistry, 8, 599-605 (2004).

44. S. Y. Shan, J. F. Yang, J. Q. Gao, W. H. Zhang, Z. H. Jin, R. Janssen, T.Ohji, "Porous Silicon Nitride Ceramics Prepared by Reduction-Nitridation of Silica,' J. Am. Ceram. Soc., 88 [9], 2594-6 (2005). 
45. A. R. Studart, W. U. T. Gonzenbach, Elena Tervoort, and Ludwig J. Gauckler, "Processing Routes to Macroporous Ceramics: A Review"; J. Am. Ceram. Soc., 89 [6] 1771-1789 (2006)

46. S. J. Powell, J. R. G. Evans, "The structure of ceramic foams prepared from polyurethane-ceramic suspensions", Materials and Manufacturing Processes, 10[4], 757-771 (1995).

47. L. Wucherer, J. C. Nino, F. Basoli, E. Traversa; "Synthesis and characterization of $\mathrm{BaTiO}_{3}$ - based foams with a controlled microstructure", Int. J. of Applied Ceramic Technology, 6[6] (2008).

48. Y. W. Lo, W. C .J. Wei, C. H. Hsueh; "Low thermal conductivity of porous A12O3 foams for SOFC insulation" Materials Chemistry and Physics, 129[1-2], 326-330 (2011).

49. A. Rainer, F. Basoli, S. Licoccia, E. Traversa, "Foaming of filled polyurethanes for fabrication of porous anode supports for intermediate temperature solid oxide fuel cells", Journal of American ceramic society, 89[6]1795-1800 (2006).

50. S. P. Jiang, W. Wang; "Fabrication and Performance of GDC-Impregnated ( $\mathrm{La}, \mathrm{Sr}) \mathrm{MnO}_{3}$ Cathodes for Intermediate Temperature Solid Oxide Fuel Cells", Journal of Electrochemical Society, 152, A1398-A1408 (2005).

51. K. Yamahara, C. P. Jacobson, S. J. Visco, L. C. De Jonghe, Solid State Ionics 176, 451-456 (2005).

52. Z. Jiang, C. Xia, F. Chen, “ Nano-structured composite cathodes for intermediate-temperature solid oxide fuel cells via an infiltration/impregnation technique”, Electrochimica Acta, 55, 3595-3605 (2010).

53. S. P. Jiang, "A review of wet impregnation-An alternative method for the fabrication of high performance and nano-structured electrodes of solid oxide fuel cells", Materials Science and Engineering A, 418, 199-210 (2006).

54. J. Fleig, "SOLID OXIDE FUEL CELL CATHODES: Polarization Mechanisms and Modeling of the Electrochemical Performance”, Annu. Rev. Mater. Res., 33, 361-82 (2006).

55. S. H. Chan, X. J. Chen, and K. A. Khor, "Cathode Micromodel of Solid Oxide Fuel Cell"; Journal of The Electrochemical Society, 151 (1), A164-A172 (2004). 
56. S. B. Adler, "Factors Governing Oxygen Reduction in Solid Oxide Fuel Cell Cathodes", Chem. Rev., 104, 4791-4843 (2004).

57. S. Wang, M. Katsuki, M. Dokiya, T. Hashimoto, "High temperature properties of $\mathrm{La} 0.6 \mathrm{Sr} 0.4 \mathrm{Co} 0.8 \mathrm{Fe} 0.2 \mathrm{O} 3$ phase structure and electrical conductivity"; Solid State Ionics, 159, $71-78$ (2003).

58. D. Beckel, U. P. Muecke, T. Gyger, G. Florey, A. Infortuna, L. J. Gauckler, "Electrochemical performance of LSCF based thin film cathodes prepared by spray pyrolysis", Solid State Ionics, 178, 407-415 (2007).

59. P. Holtappels, C. Sorof, M. C. Verbraeken, S. Rambert, U. Vogt, "Preparation of Porosity-Graded SOFC Anode Substrates. Fuel Cells", 6[2], 113-116 (2006).

60. "Electrochemical cell architecture and method of making same via controlled powder morphology”, US Patent Application 20060113034.

61. P. Colombo, "Conventional and novel processing methods for cellular ceramics”, 364[1838], 109-124 (2006).

62. A. Rainer, W. F. Basoli, S. Licoccia, E. Traversa, "Foaming of Filled Polyurethanes for Fabrication of Porous Anode Supports for Intermediate TemperatureSolid Oxide Fuel Cells”, J. Am. Ceram. Soc., 89 [6] 1795-1800 (2006).

63. A. R. Studart, U. T. Gonzenbach, E. Tervoort, L. J. Gaukler "Processing Routes to Macroporous Ceramics: A Review”, J. Am. Ceram. Soc., 89[6] 17710-1789 (2006).

64. A. Lanzini, P. Leone, P. Asinar; "Microstructural characterization of solid oxide fuel cell electrodes by image analysis technique”, Journal of Power Sources (2009).

65. K. R. Lee, S. H. Choi, J. Kim, H. W. Lee, J. H. Lee ,"Viable image analyzing method to characterize the microstructure and the properties of the Ni/YSZ cermet anode of SOFC", Journal of Power Sources, 140[2], 226-234 (2005).

66. S. M. Bae et al., "Journal of Korean Ceramic Society", 47[1], 86-91, (2010).

67. G. Impoco. "Software for the image analysis of cheese microstructure from SEM imagery" (2006) 
68. J. H. Lee, H. Moon, H. W. Lee, J. Kim, J. D. Kim, K. H. Yoon "Quantitative analysis of microstructure and its related electrical property of SOFC anode, Ni-YSZ cermet”, Solid State Ionics 148[15] (2002).

69. D. Burnat, P. Ried, P. Holtappels, A. Heel, T. Graule, D. Kata, "The Rheology of Stabilised Lanthanum Strontium Cobaltite Ferrite Nanopowders in OrganicMedium Applicable as Screen Printed SOFC Cathode Layers” (2009).

70. "Fuel Cell Materials - Products and Services", Retrieved 2012, from http://www.fuelcellmarkets.com/fuel_cell_materials_SOFC_Materials/products_and_serv ices/3,1,723,17,27956.html.

71. "Dow Polyurethanes - Surfactants Role in Foam Formulations", Retrieved $\begin{array}{llll}\text { March } & 01, & \text { 2011, from } & \text { https:/dow- }\end{array}$ answer.custhelp.com/app/answers/detail/a_id/5709/ /dow-polyurethanes---surfactantsrole-in-foam-formulations.

72. P. Mondal, D. V. Khakhar; "Hydraulic resistance of rigid polyurethane foams. II. Effect of variation of surfactant, water, and nucleating agent concentrations on foam structure and properties"; Journal of Applied Polymer Science, 93[6], 2830-2837 (2004).

73. L. W. Tai, M. M. Nasrallah, H. U. Anderson, D. M. Sparlin, S. R. Sehlin, Solid State Ionics 76, 273-283 (1995).

74. "Perovskite Materials for Solid Oxide Fuel Cell Cathodes”, United States Patent Application 20110143255.

75. J. W. Templeton, J. W. Stevenson, Z. Lu, J. S. Hardy; "Effect of A-site Non-stoichiometry on LSCF Cathodes", PNNL-20902.

76. "Polyurethanes - Learning Centre" Retrieved November 20, 2006, from http://www.wernerblank.com/polyur/learning/learning_center3.htm.

77. Y. C. TU, "POLYURETHANE FOAMS FROM NOVEL SOY-BASED POLYOLS”, PhD dissertation, University of Missouri, (2008).

78. S. Bailliez, A. Nzihou, "The kinetics of surface area reduction during isothermal sintering of hydroxyapatite adsorbent”, Chem Eng J, 98:141-152 (2004).

79. J. W. Yun, J. Han , S. Pil Yoon, S. Park , H. S. Kim , S. W. Namb, Journal of Industrial and Engineering Chemistry 17, 439-444 (2011). 
80. H. J. M. Bouwmeester, M. W. D. Otter, B. A. Boukamp, “Oxygen transport in LSCF”, Journal of Solid State Electrochemistry, 8: 599-605 (2004).

81. Jeong Woo Yun et al., Journal of Industrial and Engineering Chemistry (2011)

82. A.Esquirol et al., Journal of Electrochemical Society (2004).

83. S. P. Jiang "A review of wet impregnation-An alternative method for the fabrication of high performance and nano-structured electrodes of solid oxide fuel cells", Materials Science and Engineering: A, 418[1-2], 199-210 (2006)

84. H. Uchida, M. Yoshida, M. Watanabe, "Effect of Ionic Conductivity of Zirconia Electrolytes on the Polarization Behavior of Various Cathodes in Solid Oxide Fuel Cells"; Journal of The Electrochemical Society, 146 [1] 1-7 (1999).

85. X. Li, N. Xu, X. Zhao, K. Huang, "Performance of a commercial cathodesupported solid oxide fuel cells prepared by single-step infiltration of an ion-conducting electrocatalyst”, Journal of Power Sources, 199, 132-137 (2012).

86. V. A. C. Haanappel, D. Rutenbeck, A. Mai, S. Uhlenbruck, D. Sebold, H. Wesemeyer, B. Röwekamp, C. Tropartz, F. Tietz, "The influence of noble-metalcontaining cathodes on the electrochemical performance of anode-supported SOFCs", Journal of Power Sources 130, 119-128 (2004).

87. T. J. Huang, X. D. Shen, C. L. Chou," Characterization of Cu, Ag and Pt added LSCF and gadolinia-doped ceria as solid oxide fuel cell electrodes by temperatureprogrammed techniques”, Journal of Power Sources 187 (2009).

88. G. Impoco, "Software for Pore Detection and Analysis in Microscopy", Retrieved from http://www.impoco.it/resources_sw_pore_analysis.php (2011). 


\section{APPENDIX}

The source code of the java plug-in for ImageJ, developed by D.Gaetano Impoco, Italy for image analysis of cheese microstructure that is used in this work is as follows [88].

\section{Binarizing:}

This plugin enhances and thresholds 8-bit grey level images for particle measurements.

Copyright (c) 2006 by Gaetano Impoco (impoco@dmi.unict.it)

This plugin is free software; you can redistribute it and/or modify it under the terms of the GNU General Public License version 2 as published by the Free Software Foundation.

This program is distributed in the hope that it will be useful, but WITHOUT ANY WARRANTY; without even the implied warranty of MERCHANTABILITY or FITNESS FOR A PARTICULAR PURPOSE.

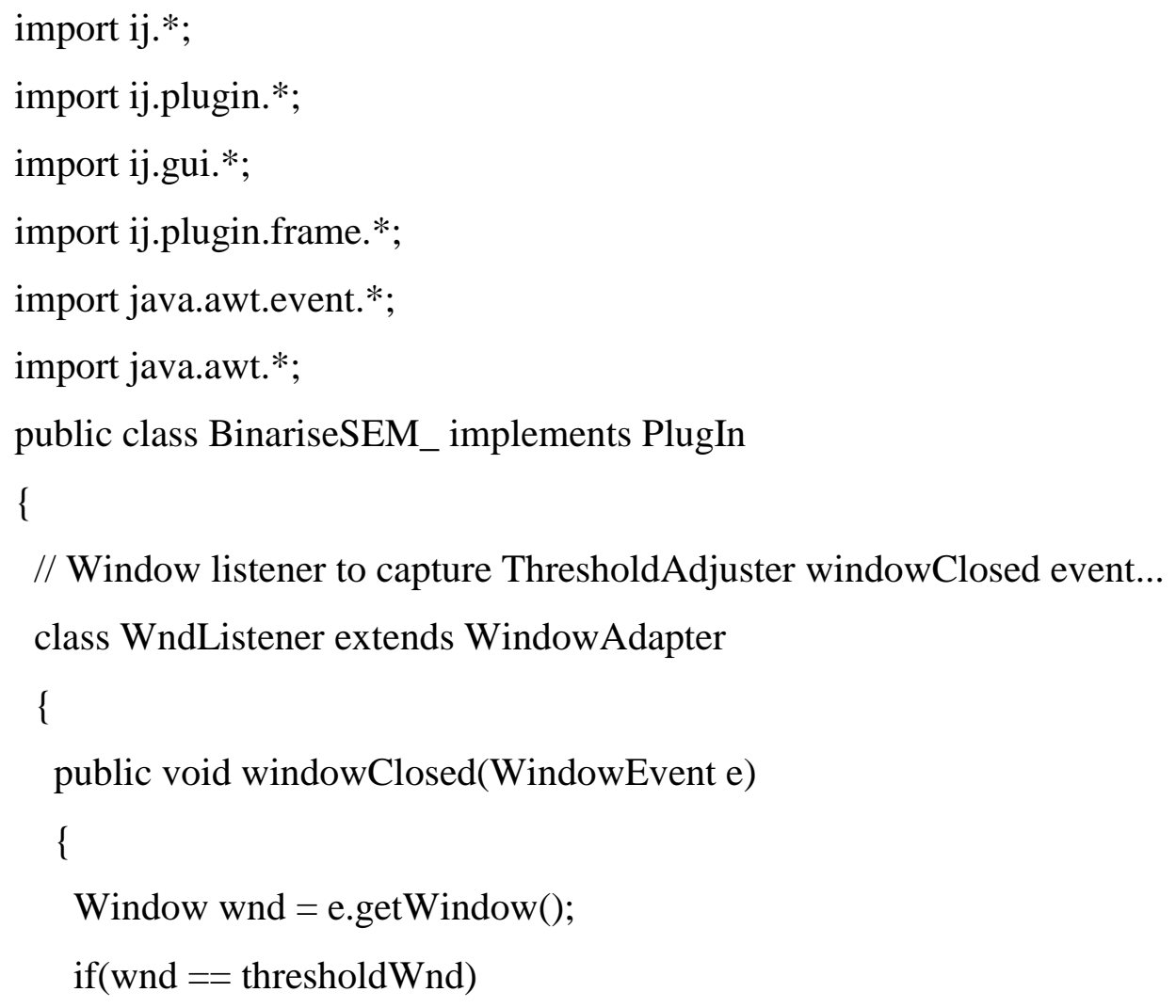




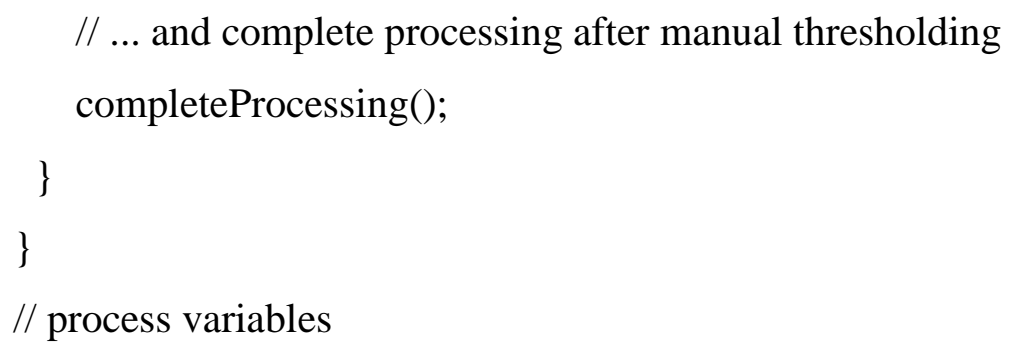

// reset threshold adjuster window

static ThresholdAdjuster thresholdWnd = null;

// parameters' dialog

public boolean showDialog() 
GenericDialog dlg = new GenericDialog("Parameters", IJ.getInstance());

// dlg.addNumericField("Radius of the Gaussian filter", radiusDia, 0); dlg.addCheckbox("Bandpass filter", bandpassDia);

// dlg.addNumericField("Filter_large structures down to x pixels", filterLargeDia, 1);

// dlg.addNumericField("Filter_small structures up to x pixels", filterSmallDia, $1)$;

// dlg.addChoice("Suppress stripes in one direction", choices, choiceDia);

// dlg.addNumericField("Tolerance of direction (\%)", toleranceDia, 1);

// dlg.addCheckbox("Autoscale after filtering", doScalingDia);

// dlg.addCheckbox("Saturate image when autoscaling", saturateDia);

// dlg.addCheckbox("Display filter", displayFilterDia);

dlg.addCheckbox("Fill inner holes", fillInnerHolesDia);

dlg.showDialog();

if(dlg.wasCanceled())

return false;

if(dlg.invalidNumber())

\{

IJ.showMessage("Error", "Invalid input Number"); return false;

\}

// radiusDia = (int)dlg.getNextNumber(); bandpassDia = dlg.getNextBoolean () ;

// filterLargeDia = dlg.getNextNumber();

$/ /$ filterSmallDia = dlg.getNextNumber();

// choiceIndex = dlg.getNextChoiceIndex () ;

// choiceDia = choices [choiceIndex];

$/ /$ toleranceDia = dlg.getNextNumber();

// doScalingDia = dlg.getNextBoolean();

// saturateDia = dlg.getNextBoolean(); 
$/ / \quad$ displayFilterDia $=$ dlg.getNextBoolean ()$;$
fillInnerHolesDia $=$ dlg.getNextBoolean();

return true;

\}

// main procedure

public void run(String arg)

\{

// show parameter dialog

if(! showDialog())

return;

// duplicate input image

IJ.run("Duplicate...", "title=Binarised_SEM_image");

// remove noise

IJ.run("Despeckle");

IJ.run("Gaussian Blur...", "radius="+radiusDia);

// bandpass filter - remove luminance gradient due to non-uniform illumination

if(bandpassDia)

\{

String parameters $=$ new String("filter_large="+filterLargeDia+"

filter_small="+filterSmallDia+" suppress="+choiceDia+" tolerance="+toleranceDia);

if $($ doScalingDia $) \quad$ parameters = parameters.concat(" autoscale");

if(saturateDia) parameters = parameters.concat(" saturate");

if(displayFilterDia) parameters = parameters.concat(" display");

IJ.run("Bandpass Filter...", parameters);

if(displayFilterDia)

IJ.run("Put Behind [tab]");

\}

// morphological dilation of pores

IJ.run("Minimum...", "radius=1"); 
// setup thresholding mode and event listeners to (manually) threshold image thresholdWnd $\quad=\quad$ (ThresholdAdjuster)

IJ.runPlugIn("ij.plugin.frame.ThresholdAdjuster", "mode=Over_Underl"");

WndListener wndEventListener $=$ new WndListener();

thresholdWnd.addWindowListener(wndEventListener);

Choice choice $=($ Choice $)$ thresholdWnd.getComponent $(5)$;

choice.select(2);

thresholdWnd.itemStateChanged(new ItemEvent(choice, 0x4000, choice, 1));

\}

// processing after manual thresholding

void completeProcessing()

\{

// morphological erosion of pore borders

IJ.run("Minimum...", "radius=1");

// fill inner matrix

if(fillInnerHolesDia)

IJ.run("Fill Holes");

\}

\}

\section{Compute Stats - for quantitative analysis}

This plugin computes number statistics and shape descriptors from a 8-bit binary image.

This plugins needs a file called "ShapeDescriptors.class" to run

Copyright (c) 2006 by Gaetano Impoco (impoco@ dmi.unict.it)

This plugin is free software; you can redistribute it and/or modify it under the terms of the GNU General Public License version 2 as published by the Free Software Foundation.

This program is distributed in the hope that it will be useful, but WITHOUT ANY WARRANTY; without even the implied warranty of MERCHANTABILITY or FITNESS FOR A PARTICULAR PURPOSE. 
import ij.*;

import ij.process.*;

import ij.gui.*;

import java.awt.*;

import ij.plugin.filter.*;

import ij.text.*;

import java.util.*;

public class ComputeStats_implements PlugInFilter

\{

// set basic colours and various default values

static int PORE_PIXEL $=255$;

static short PIXEL_BACKGROUND $=0$;

static double PIXEL_SIZE $=100.0 \mathrm{~F} ; \quad / /$ pixel size in microns, at to magnification (divide by the magnbification factor to obtain the correct size)

static double MAGNIFICATION_DEFAULT $=1000.0$;

static int paletteSize $=16$;

static byte paletteR []$=\{$ (byte) 0 , (byte) 0 , (byte) 204, (byte) 0 , (byte)

0 , (byte) 102, (byte) 255, (byte) 255, (byte) 255, (byte) 0, (byte) 255, (byte) 0 , (byte) 102, (byte) 0 , (byte) 204, (byte) 255\};

static byte paletteG[] $=\{$ (byte) $\quad 0$, (byte) 0 , (byte) 255, (byte) 102 ,

(byte) 255, (byte) 0, (byte) 0, (byte) 0, (byte) 102, (byte) 204, (byte) 204,

(byte) 255, (byte) 255, (byte) 255, (byte) 0 , (byte) 0$\}$;

static byte paletteB []$=\{$ (byte) 0 , (byte) 255, (byte) 0 , (byte) 255,

(byte) 0, (byte) 255, (byte) 204, (byte) 0, (byte) 0, (byte) 255, (byte) 0 ,

(byte) 204, (byte) 0, (byte) 102, (byte) 255, (byte) 102\};

// other global variables

DisplayList displayList; 
// display list container - exposed to the user

private class DisplayList

\{

boolean area, perimeter, maxDiameter, minDiameter, directionality, formFactor, roundness, aspectRatio;

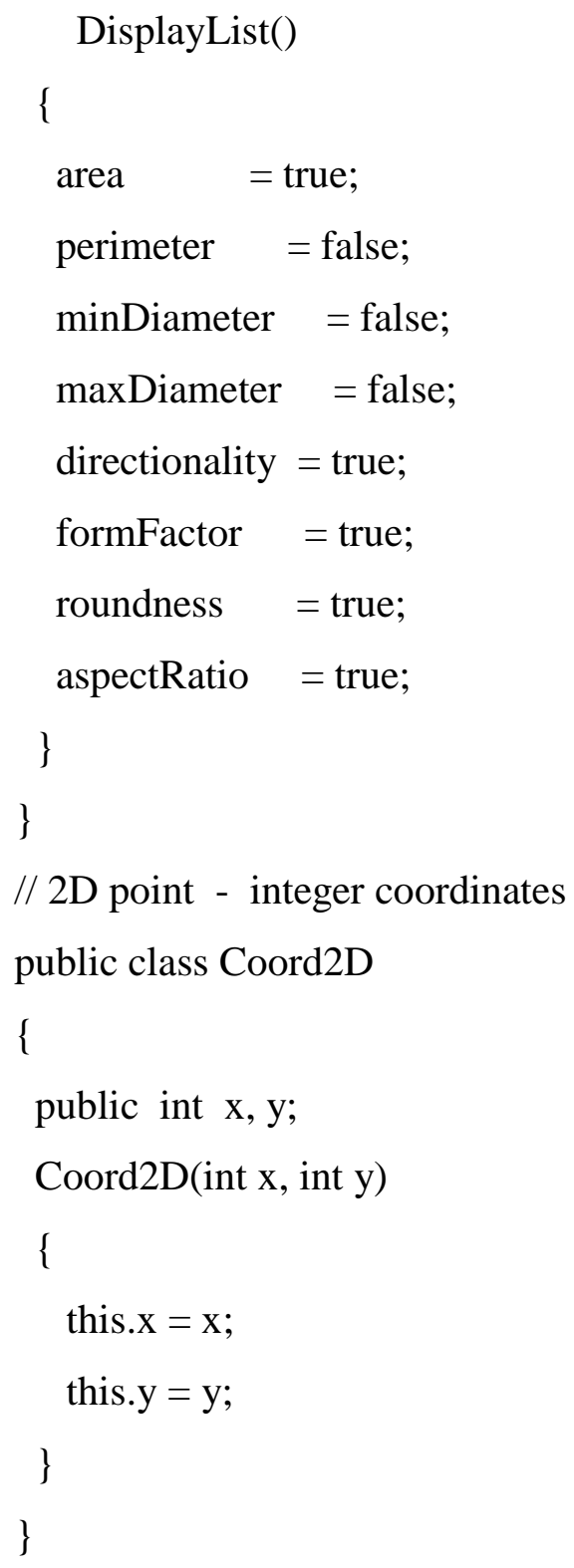




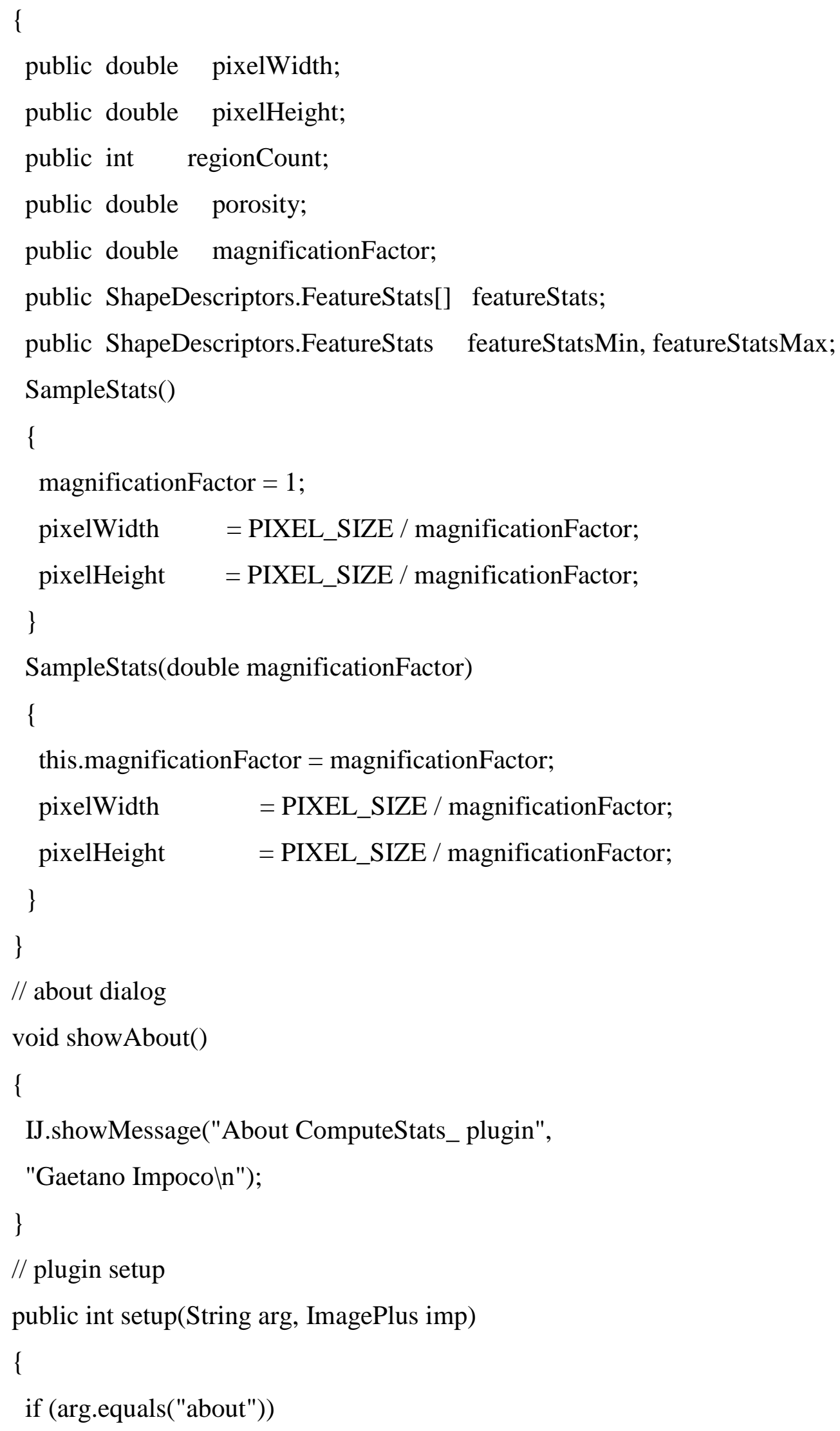




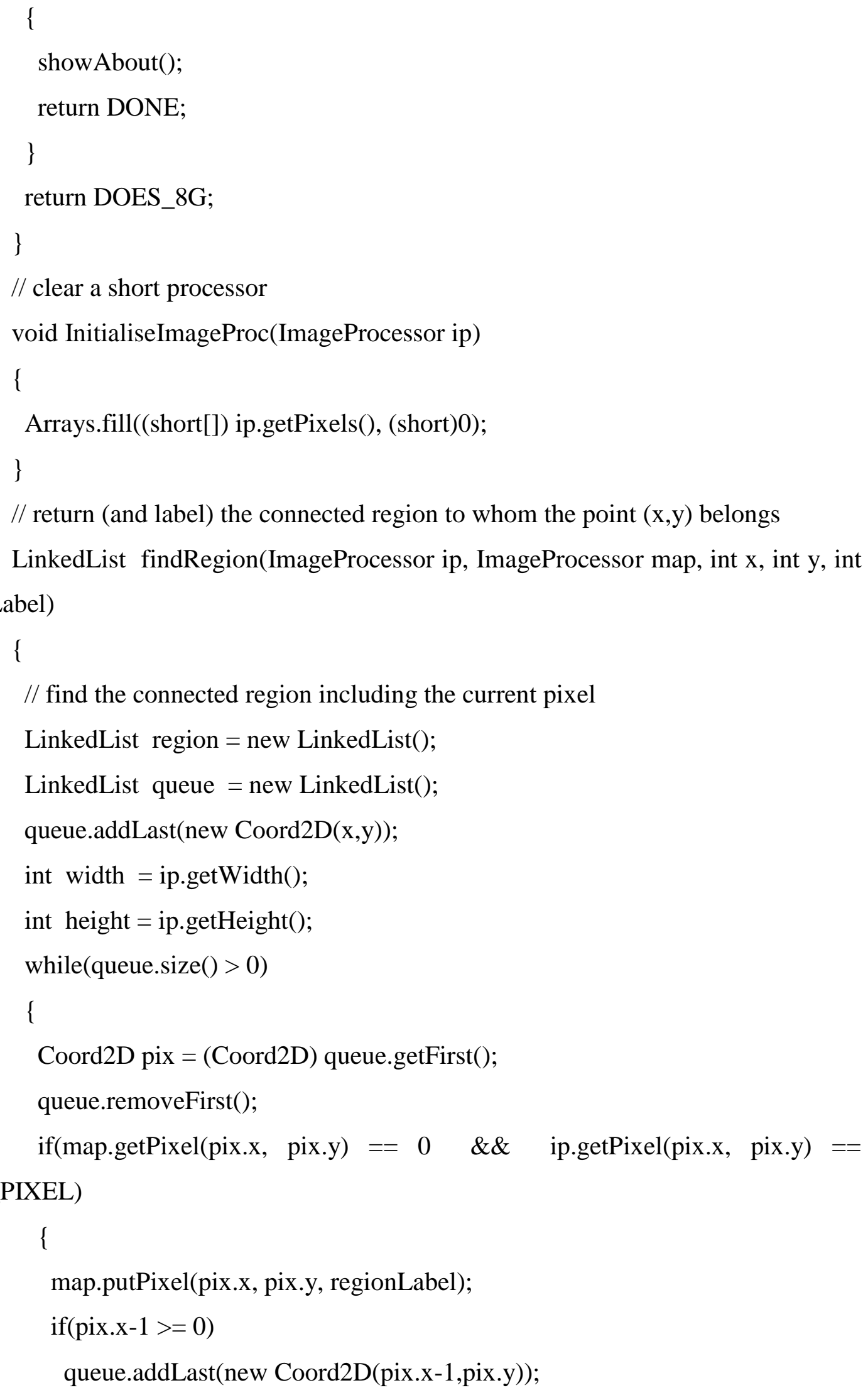




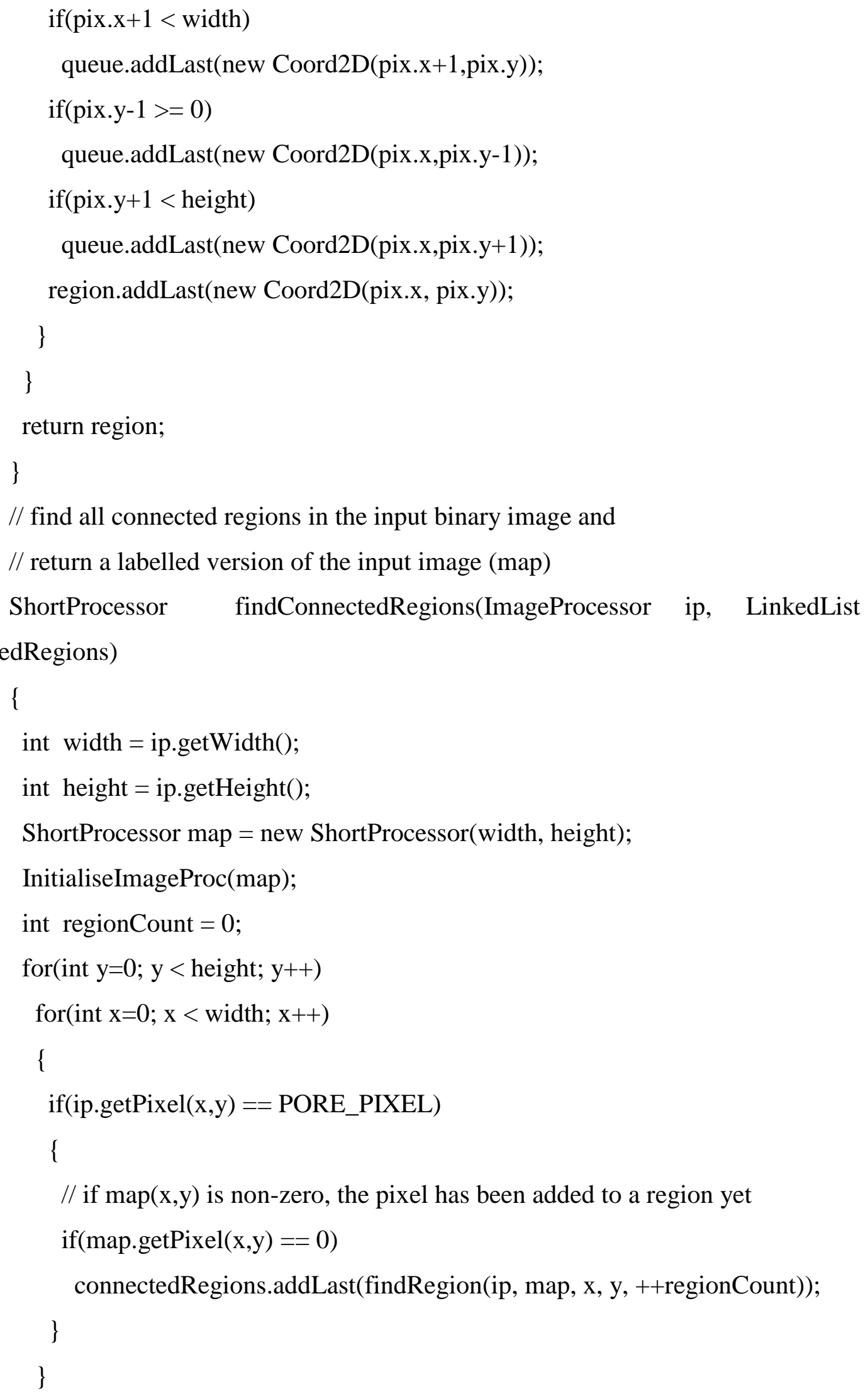




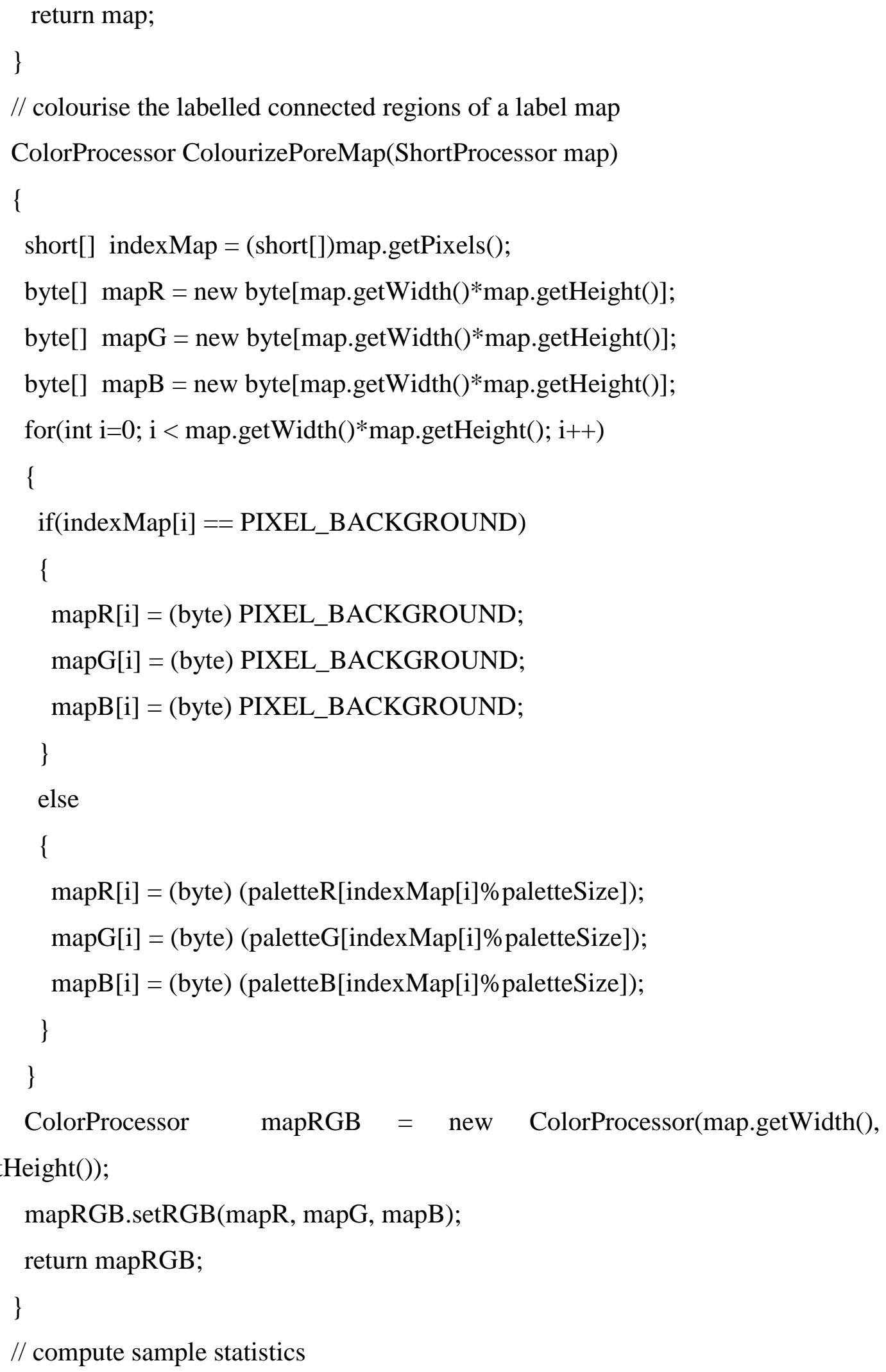


boolean computeSampleStatistics(ImageProcessor ip, LinkedList connectedRegions, SampleStats stats)

\{

stats.regionCount = connectedRegions.size () ;

double pixelArea = stats.pixelHeight*stats.pixelWidth;

double pixelSize = Math.max(stats.pixelHeight,stats.pixelWidth);

ShapeDescriptors shapeDescriptors = new ShapeDescriptors();

shapeDescriptors.setPixelDimensions(ip, pixelSize, pixelArea);

stats.featureStats = new ShapeDescriptors.FeatureStats[stats.regionCount];

stats.featureStatsMin = shapeDescriptors.new FeatureStats () ;

stats.featureStatsMax = shapeDescriptors.new FeatureStats();

stats.featureStatsMin.initAll((double)(ip.getWidth()*ip.getHeight()));

stats.featureStatsMax.initAll(0.0);

ListIterator ri=connectedRegions.listIterator(0);

for(int $\mathrm{i}=0 ; \mathrm{i}<$ stats.regionCount; $\mathrm{i}++$ )

\{

// compute feature statistics

LinkedList region $=($ LinkedList $)$ ri.$n \operatorname{ext}()$;

try $\{$ stats.featureStats[i] = shapeDescriptors.computeDescriptors(region);

\}

catch(ShapeDescriptors.DimensionsNotSetException e) \{ MessageDialog mshDlg = new MessageDialog(IJ.getImage().getWindow(), "debug", e.getMessage()); return false; \};

// porosity

double regionSize $=($ double $)$ region. $\operatorname{size}()$;

stats.porosity $+=$ regionSize;

// area

if(stats.featureStatsMin.area > stats.featureStats[i].area)

stats.featureStatsMin.area = stats.featureStats[i].area;

if(stats.featureStatsMax.area < stats.featureStats[i].area) 
stats.featureStatsMax.area $=$ stats.featureStats[i].area;

// perimeter

if(stats.featureStatsMin.perimeter > stats.featureStats[i].perimeter) stats.featureStatsMin.perimeter = stats.featureStats[i].perimeter;

if(stats.featureStatsMax.perimeter < stats.featureStats[i].perimeter) stats.featureStatsMax.perimeter = stats.featureStats[i].perimeter; // diameter

if(stats.featureStatsMin.minDiameter > stats.featureStats[i].minDiameter) stats.featureStatsMin.minDiameter $=$ stats.featureStats[i].minDiameter; if(stats.featureStatsMax.minDiameter < stats.featureStats[i].minDiameter) stats.featureStatsMax.minDiameter $=$ stats.featureStats[i].minDiameter; if(stats.featureStatsMin.maxDiameter > stats.featureStats[i].maxDiameter) stats.featureStatsMin.maxDiameter = stats.featureStats[i].maxDiameter; if(stats.featureStatsMax.maxDiameter < stats.featureStats[i].maxDiameter) stats.featureStatsMax.maxDiameter $=$ stats.featureStats[i] $\cdot$ maxDiameter; // directionality

if(stats.featureStatsMin.directionality > stats.featureStats[i].directionality) stats.featureStatsMin.directionality = stats.featureStats[i].directionality; if(stats.featureStatsMax.directionality < stats.featureStats[i].directionality) stats.featureStatsMax.directionality = stats.featureStats[i].directionality; // Form factor

if(stats.featureStatsMin.formFactor > stats.featureStats[i].formFactor) stats.featureStatsMin.formFactor $=$ stats.featureStats[i].formFactor; if(stats.featureStatsMax.formFactor < stats.featureStats[i].formFactor) stats.featureStatsMax.formFactor = stats.featureStats[i].formFactor; // roundness

if(stats.featureStatsMin.roundness > stats.featureStats[i].roundness) stats.featureStatsMin.roundness = stats.featureStats[i].roundness; if(stats.featureStatsMax.roundness < stats.featureStats[i].roundness) stats.featureStatsMax.roundness = stats.featureStats[i].roundness; // aspect ratio 
// create a (white) blank image

int width $=400$;

int height $=300$;

ByteProcessor rosePlotImg = new ByteProcessor(width,height);

rosePlotImg.setBackgroundValue(255.0);

rosePlotImg.setValue(255.0); // WHITE

rosePlotImg.fill();

// draw the plot

rosePlotImg.setColor(0);

int $\max =0$;

for(int $\mathrm{i}=0 ; \mathrm{i}<\mathrm{nBins} ; \mathrm{i}++)$

if $(\max <\operatorname{histogram}[\mathrm{i}]) \quad \max =\operatorname{histogram}[\mathrm{i}]$;

int $\mathrm{Cx}=$ width $/ 2$;

int $\mathrm{Cy}=$ height $/ 2$;

double factor $=($ double $)$ Math.min $($ width,height $) / 2.0 /($ double $) \max$;

Polygon roseBoundary = new Polygon () ;

int lastX = (int) $($ Math.cos $(\operatorname{minVal}) *($ double $)$ histogram $[0] *$ factor $)+\mathrm{Cx}$;

int last $\mathrm{Y}=($ int $)($ Math.sin $(\operatorname{minVal}) *($ double $)$ histogram $[0] *$ factor $)+\mathrm{Cy}$;

roseBoundary.addPoint(lastX, lastY);

for(int bin=1; bin < nBins; bin++)

\{

double angle $=(($ double $)$ bin $/($ double $) n B i n s) *(\operatorname{maxVal}-\operatorname{minVal})+\operatorname{minVal}$;

int $\mathrm{x}=($ int $)($ Math.cos(angle) $*$ (double)histogram[bin] $*$ factor $)+\mathrm{Cx}$;

int $\mathrm{y}=($ int $)($ Math.sin $($ angle $) *($ double $)$ histogram [bin] $*$ factor $)+\mathrm{Cy}$;

rosePlotImg.drawLine(lastX, lastY, $\mathrm{x}, \mathrm{y})$;

roseBoundary.addPoint( $\mathrm{x}, \mathrm{y})$;

last $\mathrm{X}=\mathrm{x}$;

lastY = y;

\} 
for(int bin=1; bin < nBins; bin++)

\{

double angle $=(($ double $)$ bin $/($ double $) n B i n s) *(\max V a l-m i n V a l)+\operatorname{minVal}$

+ Math.PI;

int $\mathrm{x}=($ int $)($ Math.cos $($ angle $) *($ double $)$ histogram $[$ bin $] *$ factor $)+\mathrm{Cx}$;

int $\mathrm{y}=$ (int) $($ Math.sin(angle) $*$ (double)histogram[bin] $*$ factor $)+\mathrm{Cy}$;

rosePlotImg.drawLine(lastX, lastY, $\mathrm{x}, \mathrm{y})$;

roseBoundary.addPoint $(\mathrm{x}, \mathrm{y})$;

last $\mathrm{X}=\mathrm{x}$;

lastY = y;

\}

// fill the inner of the rose

rosePlotImg.setColor(128);

rosePlotImg.fillPolygon(roseBoundary);

// draw axes

rosePlotImg.setColor(0);

rosePlotImg.drawLine $(\mathrm{Cx}, 0, \mathrm{Cx}$, height-1);

rosePlotImg.drawLine( $0, \mathrm{Cy}$, width-1, Cy);

// display window

ImageWindow imgWindow $=$ new ImageWindow(new ImagePlus(wndTitle, rosePlotImg));

\}

// display the histograms and rose plots of the selected descriptors (i.e., those in the display list)

void displayHistograms(DisplayList dispList, ShapeDescriptors.FeatureStats[] featureStats, int nBins, ShapeDescriptors.FeatureStats featureStatsMin, ShapeDescriptors.FeatureStats featureStatsMax)

\{

double data[] = new double[featureStats.length]; 
if(dispList.area)

\{

for(int $\mathrm{i}=0 ; \mathrm{i}<$ featureStats.length; $i++)$

data[i] = featureStats[i] $\cdot$ area;

HistogramWindow histWin $=$ new HistogramWindow("Pore Area Distribution (squared microns)", new ImagePlus("", new FloatProcessor(featureStats.length, 1, data)), nBins, featureStatsMin.area, featureStatsMax.area);

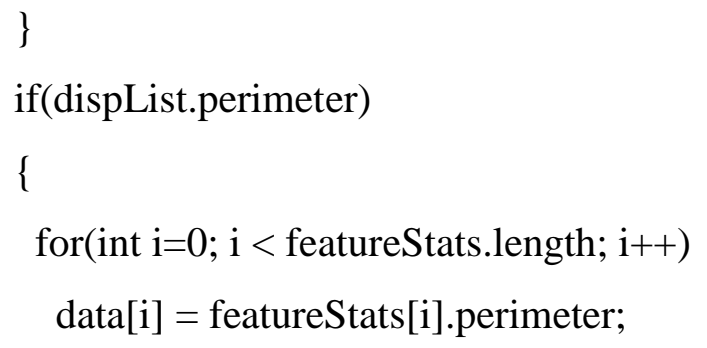

HistogramWindow histWin $=$ new HistogramWindow("Pore Perimeter Distribution (microns)", new ImagePlus("', new FloatProcessor(featureStats.length, 1, data)), nBins, featureStatsMin.perimeter, featureStatsMax.perimeter);

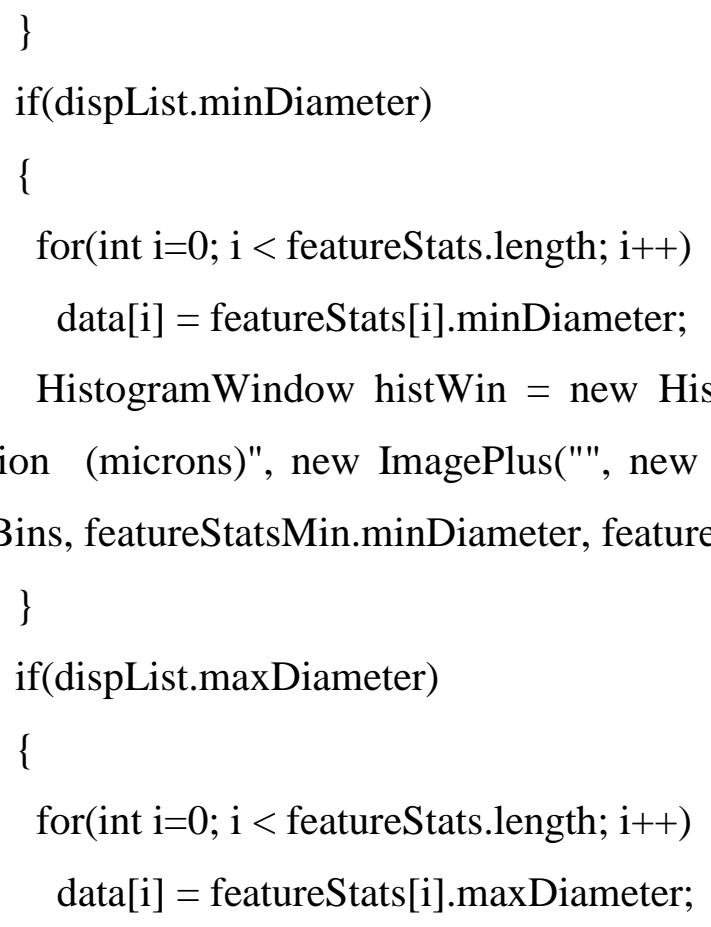
data)), nBins, featureStatsMin.minDiameter, featureStatsMax.minDiameter); 
HistogramWindow histWin $=$ new HistogramWindow("Pore Max Diameter Distribution (microns)", new ImagePlus("', new FloatProcessor(featureStats.length, 1, data)), nBins, featureStatsMin.maxDiameter, featureStatsMax.maxDiameter);

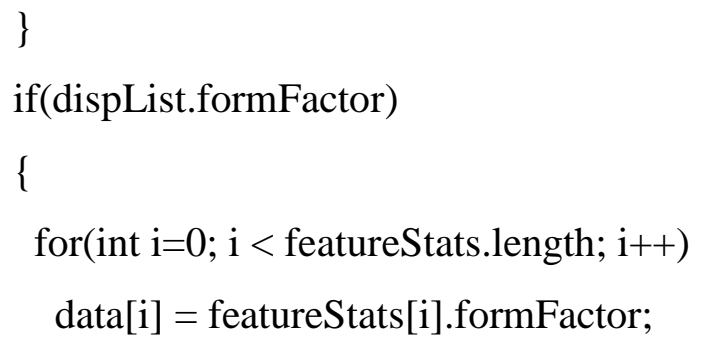
Distribution", new ImagePlus("", new FloatProcessor(featureStats.length, 1, data)), nBins, featureStatsMin.formFactor, featureStatsMax.formFactor);

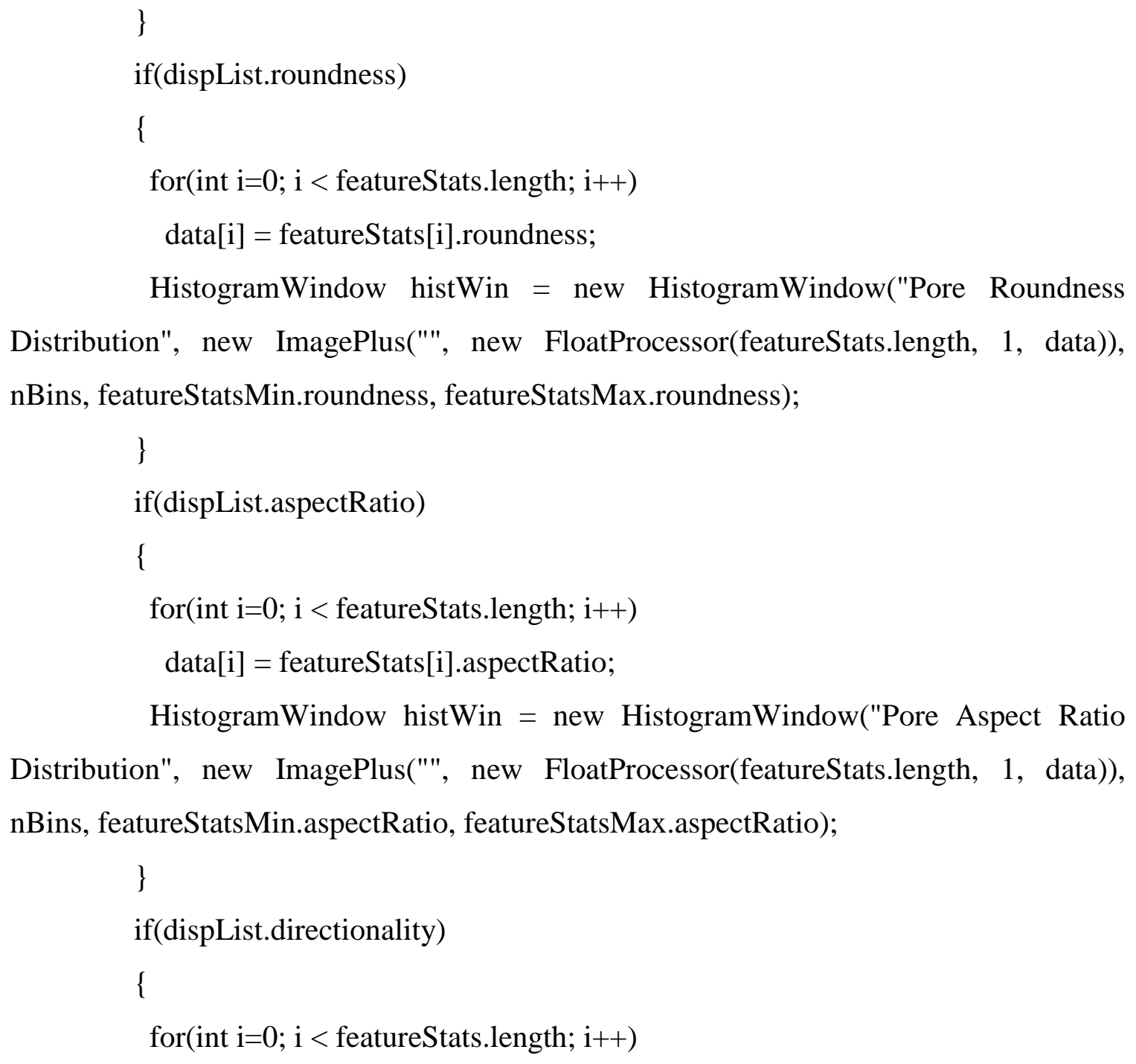
nBins, featureStatsMin.roundness, featureStatsMax.roundness); 


$$
\text { data[i] = featureStats[i].directionality; }
$$

HistogramWindow histWin = new HistogramWindow("Pore Directionality Distribution", new ImagePlus("", new FloatProcessor(featureStats.length, 1, data)), nBins, featureStatsMin.directionality, featureStatsMax.directionality);

// drawRosePlot("Pore Directionality Distribution", computeHistogram(data, featureStats.length, nBins, featureStatsMin.directionality, featureStatsMax.directionality), nBins, featureStatsMin.directionality, featureStatsMax.directionality);

drawRosePlot("Pore Directionality Distribution", histWin.getHistogram(), nBins, featureStatsMin.directionality, featureStatsMax.directionality);

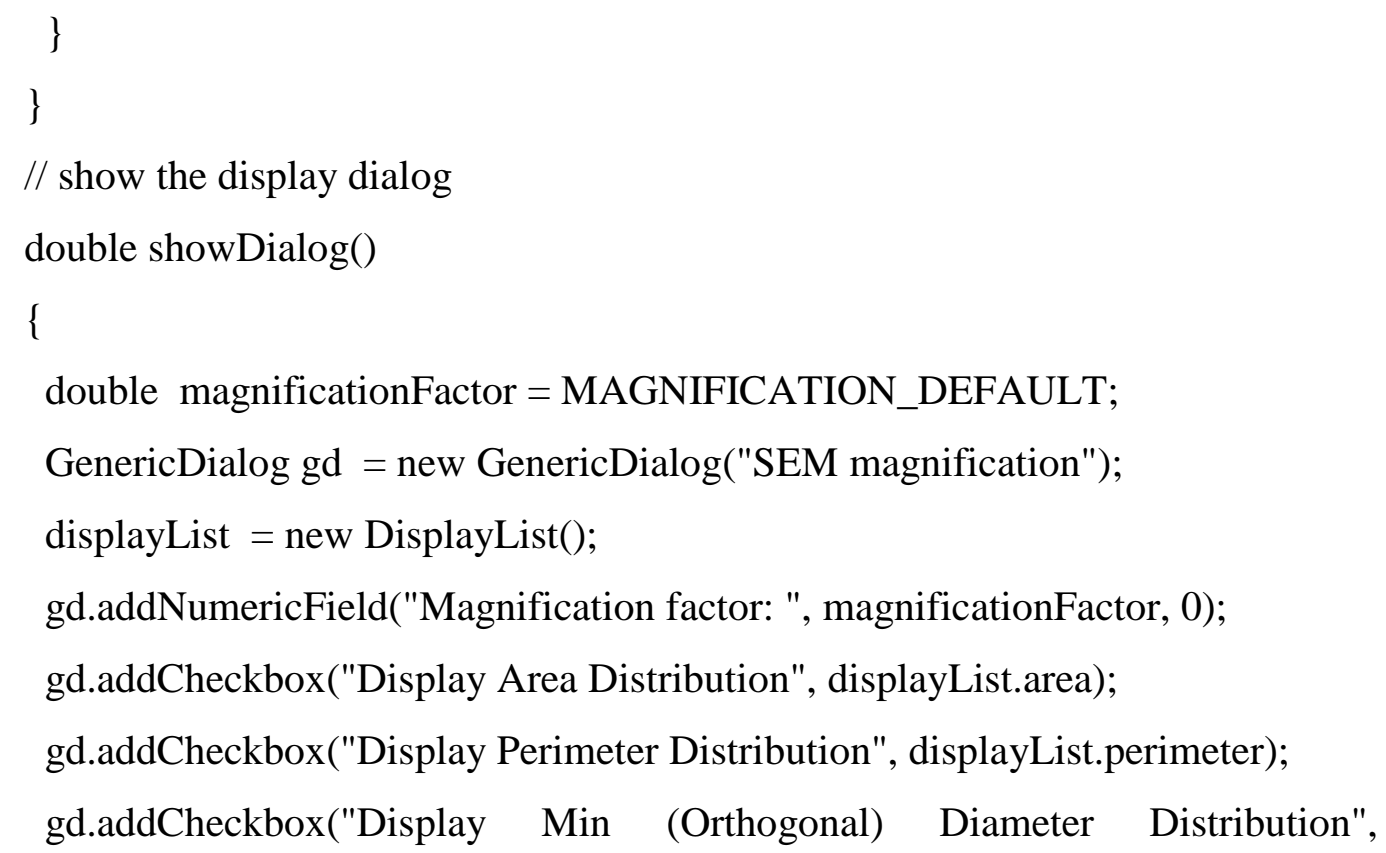




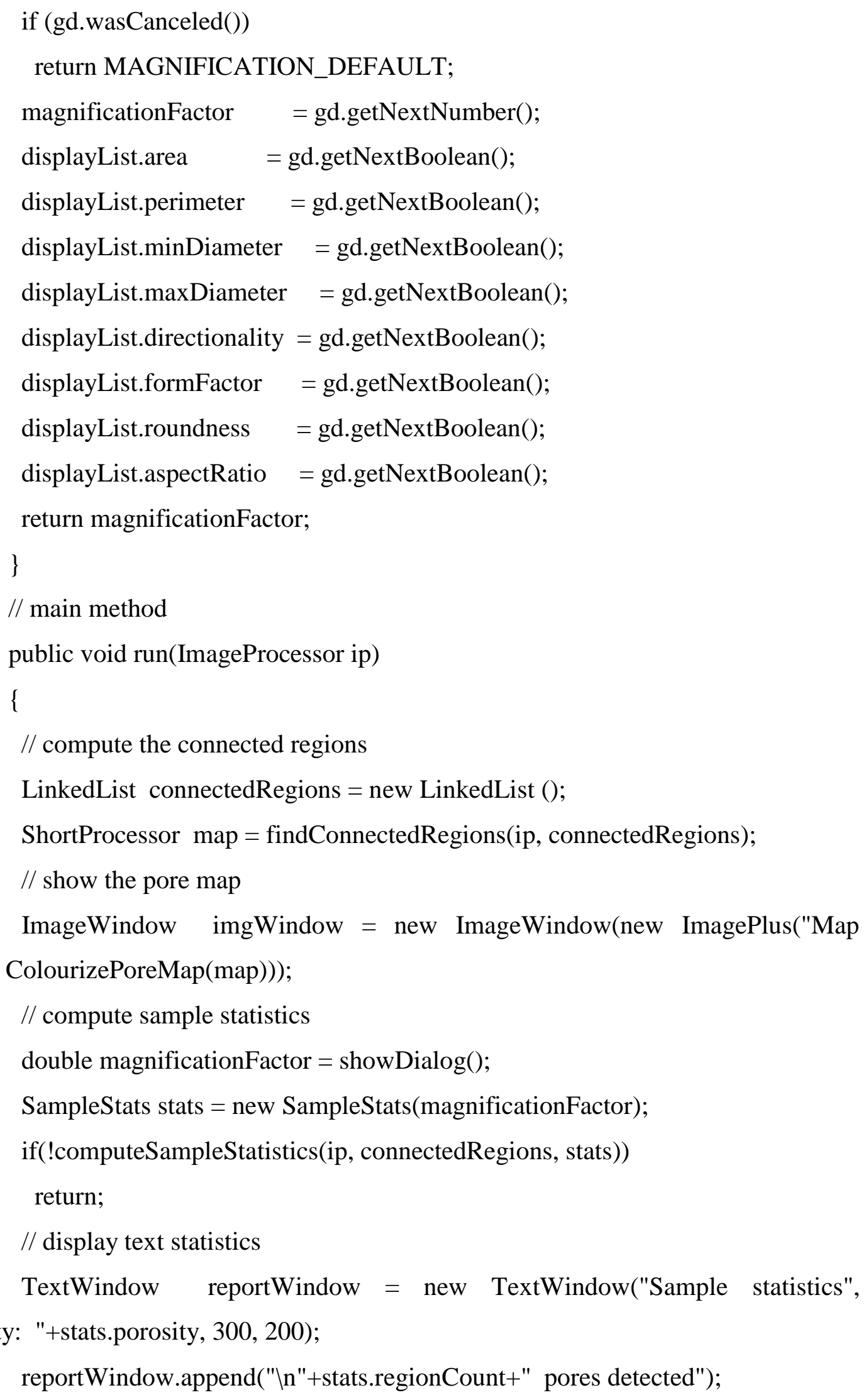


// show histograms

displayHistograms(displayList, stats.featureStats,

Math.max(stats.regionCount/20,150), stats.featureStatsMin, stats.featureStatsMax);

\}

\} 